\title{
Recherches préparatoires à une histoire de l'épistémologie des sciences humaines
}

\section{Giovanni Busino}

\section{(2) OpenEdition}

\section{Journals}

Édition électronique

URL : http://journals.openedition.org/ress/325

DOI : $10.4000 /$ ress.325

ISSN : 1663-4446

Éditeur

Librairie Droz

Édition imprimée

Date de publication : 1 août 2005

Pagination : 83-162

ISBN : 2-600-01027-0

ISSN : 0048-8046

Référence électronique

Giovanni Busino, «Recherches préparatoires à une histoire de l'épistémologie des sciences humaines ", Revue européenne des sciences sociales [En ligne], XLIII-132 | 2005, mis en ligne le 30 octobre 2009, consulté le 01 mai 2019. URL : http://journals.openedition.org/ress/325 ; DOI : 10.4000/ress.325 


\section{Giovanni BUSINO}

\section{RECHERCHES PRÉPARATOIRES À UNE HISTOIRE DE L'ÉPISTÉMOLOGIE DES SCIENCES HUMAINES}

\section{DE LA PREUVE DANS LES SCIENCES HUMAINES ${ }^{1}$}

Depuis l'époque des penseurs Grecs, la notion de preuve demeure au centre de la réflexion des épistémologues et des historiens des sciences. Des nombreux livres en retracent l'historique, informent sur les problématiques du passé et du présent, d'Aristote à Wittgenstein, de Kuhn à Stich. Bien que ces travaux n'aient exercé qu'une influence négligeable sur les praticiens des sciences humaines, il est profitable de prendre pour point de départ les définitions de la preuve qu'ils attribuent aux sciences «dures » et à celles dites «molles»

Dans les sciences «dures » (formelles et expérimentales), la preuve est un test, un procédé d'évaluation, de validité, une construction inférentielle présumée correcte si les prémisses initiales impliquent la conclusion. La formalisation de la vérification du test est une démonstration, c'est-à-dire une suite finie de propositions produites par une règle de déduction. La démonstration «prouve » l'absence d'incohérences dans le procédé utilisé. La preuve est déductive si, en vertu de règles logiques, l'aboutissement est une conclusion nécessaire; elle est inductive, probabiliste, ou stochastique, si établie au moyen des critères statistiques.Pour faire court, prouver signifie chercher et formaliser une relation entre un conséquent et un antécédent supposé, expliquer revient à chercher l'antécédent d'un conséquent donné tandis que conclure équivaut à déduire les conséquences de l'antécédent. Entre la preuve et la démonstration il y a, en principe, une équivalence ${ }^{2}$.

'Texte révisé d'une leçon donnée à l'Université de Genève, Groupe d'étude d'histoire moderne et contemporaine présidé par le professeur Michel Porret. Pour des plus amples développements, je me permets de renvoyer à mon article La preuve dans les sciences sociales, «Revue européenne des sciences sociales », XLI, 2003, n. 128, pp. 11-61.

Selon le professeur Jean-Blaise Grize (lettre du 2 mars 2004) il faut distinguer les preuves des démonstrations: «[...] une preuve me semble toujours être pour quelqu'un tandis qu'une démonstration est de quelque chose. La première est un raisonnement, la seconde un calcul et je ne vois pas en quoi se justifierait la réduction du raisonnement au calcul. [...] Il découle de là l'importance décisive des croyances, l'obligation de l'accord des esprits et la place du vraisemblable à côté de celle du vrai.» «Je souscris à la distinction qu'opère Dewey entre des lois universelles et des lois générales. Les premières ne font que dénoter les objets de quelques modèles, elles fournissent des raisons et sont non existentielles; les secondes traitent des choses du monde, elles fournissent des causes et sont de nature existentielles. Puisque les sciences de l'homme traitent de l'homme et que nous existons (je pense, donc je suis), c'est à la recherche de lois générales qu'il faut se consacrer. L'activité de modélisation l'emporte en importance sur les modèles eux-mêmes et je doute de l'intérêt réel qu'il y a à 'produire des propositions universelles'». 
Dans les sciences de l'homme et de la société, de l'anthropologie à la sociologie, de la psychologie sociale à histoire, dans toutes les disciplines où prévalent les témoignages recoupés, les interprétations argumentées, les mises en parallèle de savoirs contextuels, la définition de la notion de preuve est très compliquée. Certes, les disciplines sociales font appel à l'induction, cependant entre les chercheurs il n'y a pas de consensus sur sa fonction dans le raisonnement, sur les propositions inductrices au moyen desquelles on remonte d'un cas singulier à une généralisation. Ce désaccord sape les fondements mêmes de la démarche scientifique. Dès lors, pas de règles communes, pas de langage unique, aucune cumulativité, échanges impraticables entre théories concurrentes, impossibilité de tester les hypothèses au travers d'expérimentations ou d'observations.

Prenons, à titre d'exemplification, les travaux de Pierre Bourdieu sur les systèmes scolaires. Pour ce chercheur les statistiques prouvent (une fois défalqué l'effet de sur-sélection déterminé par la surreprésentation des élèves des classes sociales populaires dans les filières d'excellence) que l'école réserve les filières longues aux élèves des classes sociales aisées, qu'elle favorise immanquablement les déjà favorisés, les héritiers. La preuve serait donnée par le fait que le système scolaire ne peut que reproduire l'habitus de classe, conçu comme une combinaison de cadres de références, de stratégies utilisant les repères de ces cadres, de modes de mise en pratique inconscients de ces mêmes cadres. Pour Raymond Boudon, au contraire, puisque les parents tiennent compte des coûts croissants des études longues et des probabilités que la poursuite du cursus scolaire pourrait assurer à l'enfant un meilleur avenir, la preuve est donnée par l'effet cumulatif de décisions rationnelles prises sous les contraintes des revenus disponibles.

Ces deux éminents chercheurs trouvent dans les mêmes statistiques des preuves antithétiques de comportements aux fonctions impénétrables, mystérieuses. Pourquoi? Parce que ces preuves ne disent rien sur le comment les parents changent ou non de type de choix, sur le comment ils ajustent, en fonction d'interactions locales, leurs décisions. En d'autres termes, ni l'habitus ni l'effet cumulatif des décisions par les acteurs eux-mêmes ne sont mis à l'épreuve. Ils existent parce que les chercheurs présument de les avoir décelés, parce qu'ils supposent que des «objets sociaux» sont aussi réels que les objets physiques bien qu'ils soient construit par un modèle, par une des opérations de conceptualisation.

Nous savons que les conduites individuelles et les actions collectives, les représentations sociales sont variables, indéterminées, adaptatrices, peu uniformes, diversement standardisées. Cependant nous continuons à utiliser des théories qui présupposent que la régularité des conduites est déterminée par une structure et des fonctions sous-jacentes. Dans un situé au-dessous se trouveraient réunis des éléments hétéroclites (la tradition, les normes, les règles, les habitudes, les dispositions, les routines, les capacités, l'habitus), des savoirs non propositionnels (connaissances pratiques, savoir-faire, savoirs tacites), des significations et des fonctions plus ou moins indescriptibles, voire invérifiables. Une telle présomption transforme, automatiquement, la relation idéelle entre concepts en une relation empirique entre indicateurs, elle nous fait utiliser la preuve conditionnelle et partielle dépouillée de la double adéquation (significative et causale). Précisément pour cette raison, la preuve par la cohérence est toujours entachée des apories de la circularité logique. En somme, dans les disciplines socio-historiques la preuve ne renvoie pas aux procédures pour l'établissement d'une connaissance 
valide. Dans ces disciplines il y a disparité et hétérogénéité de pratiques en matière de preuve (méthode standard, démonstration, expérimentation, validation empirique, formation des hypothèses, sélection des déterminations susceptibles de réplication et de vérification) et bien évidemment une incompatibilité de vues sur les façons de pratiquer l'explication et la compréhension, sur le statut scientifique de ces notions.

Maintenant j'aimerais illustrer, à l'aide de quelques exemples, ces remarques peut-être trop abstraites et télégraphiques.

Dans les disciplines de la Préhistoire (la Paléontologie, la Paléoanthropologie, l'Archéologie préhistorique, la Paléoethnologie et autres) le rassemblement des éléments d'une preuve est limité étant donné que le nombre de variables à déterminer reste inconnu. Ces disciplines pratiquent le raisonnement régressif, des conséquences aux principes. Les documents, les témoignages, indirectes, incomplets, incertains, douteux, n'apportent que des informations restreintes, les pièces comparatives sont fragmentaires, disparates, parfois même inexistantes. L'interprétation des données disponibles doit s'appuyer, pour bâtir une preuve, sur ce qui n'est pas donné, sur des conjectures. Les critères pour déterminer la pertinence des informations retenues et des langages décrivant la logique des opérations effectuées, restent indéterminables et réfractaires aux réplications des procédures. La connaissance indirecte par les traces, par leur recoupement, par la convergence des indices, par le raisonnement des effets aux causes, permet, dans un certain nombre de cas, de remonter à l'événement. Il n'en reste pas moins que cette connaissance n'arrive pas à effacer les suppositions, à combler les lacunes, à hiérarchiser les causes alternatives ou possibles, à pondérer la documentation, à donner une évaluation critique des informations. Une tache noire dans un terrain peut indiquer la pourriture d'un poteau qui était là, au moment de commencer les fouilles, mais également d'autres choses que nous ignorons et que nous ne savons ni pouvons prendre en considération. Les chercheurs transforment des informations imprécises en preuve d'où ils soutirent une hypothèse transformée aussitôt en un fait établi, ou en d'autres hypothèses qui à leur tour servent de plate-forme à des nouvelles hypothèses. Les lacunes sont comblées au moyen d'extrapolations et de généralisations. Avec le stratagème bien connu en dialectique éristique, d'un fait notoire certifié dans un contexte on tire subséquemment une induction que l'on fait valoir ailleurs. L'application de nos savoirs actuels (l'archéométrie, la stratigraphie, les repérages en plan et niveau, les méthodes physiques) à des sociétés disparues à jamais et sur lesquelles il n'y a que des données incertaines ou impressionnistes, rend le raisonnement causal fallacieux. Parfois on construit un modèle, à l'aide des données générales sur les sociétés humaines connues et réputées proches de celle qu'on est en train d'étudier, et on essaie ensuite de le vérifier avec les archives archéologiques disponibles. On projette ainsi sur le document découvert et sur sa place dans l'organisation spatiale, l'image de ce que l'on connaît déjà. Le préhistorien ne cesse de concevoir la vie des hommes préhistoriques comme étant proche de celle des sociétés primitives des ethnologues et, pour les périodes les plus anciennes, proche de celle des grands singes anthropoïdes.

Et c'est ainsi que l'on passe de l'homme de Neandertal à l'homo sapiens Idàltu, de la théorie que l'homo sapiens est apparu en plusieurs endroits du monde au même moment à une autre affirmant qu'il a son origine en Afrique. La preuve 
serait donnée par des subtiles variations morphologiques (longueur et robustesse) des restes d'un crâne déterré dans la région d'Afar, en Ethiopie, et que les isotopes d'argon datent de 150.000/160.000 ans. Les fortes imprécisions à propos de la datation, la mauvaise qualité des restes déterrés, l'impossibilité de les comparer avec d'autres matériaux proches, ne semblent pas trop perturber la certitude des chercheurs.

Les polémiques à propos du crâne de Toumaï, vieux de sept millions d'années, retrouvé dans le désert Tchadien, qualifié par certains du plus ancien ancêtre de l'humanité, par d'autres paléoanthropologues le plus vieux des préhumains connus, et par certains des restes d'un grand singe, ces polémiques sont bien connues $^{3}$. Sur quelle base considérer appartenir au même individu deux fossiles retrouvés à 105 mètres environs du site où avait été découvert, six mois auparavant, le crâne de Toumaï? Et c'est ainsi aussi que le déclin et la disparition de la civilisation Mayas auraient été déterminés par des perturbations climatiques, par les sécheresses qui auraient épuisées les eaux des réservoirs souterrains des cités et déstabilisé le pouvoir politique chargé des approvisionnements. Dès lors les Mayas auraient abandonné, en plusieurs phases, notamment dans les années 810 , 860 et 910 de notre ère, leurs centres religieux du Sud. La preuve de cette affirmation serait donnée par la reconstitution, au moyen d'analyses géologiques et chimiques, des régimes des pluies et de l'intensité des précipitations, en bref par les perturbations climatiques et par le rôle de l'eau dans la survie des hommes et des sociétés.

L'accord entre les chercheurs est utilisé aussi comme étant une preuve. Le recours régulier aux prejudicia (thèses identiques ou analogues acceptées par des savants reconnus), aux rumores atque fama («on retient communément», «il est désormais assuré », «il est bien établi », etc.) est un usage courant dans ce genre de recherches.

Dans ces disciplines on trouve, on découvre, on collecte, on analyse et on classe des données disparates selon des soi-disant critères de nature inductive. Pour ceci faire, il faut formuler une hypothèse reliant les données entre elles afin de produire une représentation cohérente. Il s'agit de formuler une abduction, un type d'inférence (le signe, l'objet, l'interprétant) qui fournit une hypothèse explicative d'un ensemble de faits. La prémisse majeure du syllogisme est certaine, mais la mineure est probable, donc l'inférence peut ne pas être vraie.

Partir d'un indice pour arriver à des présupposés oblige à donner du sens aux données moyennant des conjectures, des prophéties rétrospectives, des abductions non codifiées, non basées sur un savoir typologique mais uniquement sur des symptômes et sur des indices ambigus. En établissant des analogies entre des observations faites dans des contextes différents mais jugés ou réputés apparentés à un titre quelconque, le chercheur doit jouer sur des relations entre les objets d'une part et entre les contextes de l'autre afin d'avoir des typologies génératrices d'assertions. Le statut de ces assertions reste néanmoins hypothétique; en conséquence, ces assertions ne constituent pas une preuve, ni validée ni validable.

Le cas de la démographie est encore plus frappant. Cette discipline affiche une confiance absolue dans l'évidence des déductions mathématiques et/ou dans l'ob-

3 Michel Brunet, Toumaï, à la découverte de l' homme, Paris, La Table ronde, 2003. 
servation statistique des données. La preuve de la baisse de la fécondité observée dans les années '70 est prouvée, par exemple, par les mesures des flux et des stocks (décès, naissances, etc.) des populations et par le postulat que les femmes ont ou non une conception séquentielle de la fécondité. Là où les conduites en matière de procréation et de contraception ne sont pas inscrites dans la logique d'une structure de parenté et de la prise en charge élargie des enfants, on va chercher les preuves ailleurs. Et on les trouve alors dans la diffusion de la contraception, ou dans le travail professionnel de la femme, ou dans la législation égalitaire entre les sexe, ou dans les divorces più faciles, ou dans les nouveaux modèles familiaux, ou dans la partage des tâches, ou dans les contraintes sociales ou, enfin, dans les transformations radicales induites par la globalisation des styles de vie. Malgré les indices de fécondité et de mortalité, malgré les comparaisons, malgré les raisonnements sur les similitudes et sur les différences entre des facteurs exogènes, les preuves apportées manquent de consistance, elles ne disent rien sur pourquoi la fécondité ici s'effondre et là remonte. Le recours aux déterminants économiques, ou biens à d'autres déterminations, ne fait que renvoyer au prouver d'autres comportements, eux-mêmes non prouvés.

Il en va de même pour l'augmentation des femmes dans la population active. S'il s'agit d'une augmentation de la proportion des femmes dans cette population, comment expliquer ce changement par rapport à la situation des années '50? Fautil l'expliquer par la structure par âge de la population active, par des meilleures perspectives professionnelles conquises par les femmes, ou bien par une modification de nos attentes sociales? Ou encore par les répercussions économiques, directes ou indirectes, sur le niveau de vie des femmes et de leurs familles?

Il n'y a pas de réponses plausibles à ces questions, pas non plus de preuves pour les argumenter rigoureusement. Il faut bien reconnaître que les disciplines socio-historiques ne disposent d'aucun critère absolu d'évaluation des théories. Ces sciences réduisent la rationalité au principe de raison suffisante dont la causalité n'est qu'un des aspects et l'explication une implication dans le fait. Rares sont les démonstrations en tant que suites finies de propositions déduites par la règle de déduction à partir d'axiomes et de propositions déjà démontrés ${ }^{4}$.

Mais pourquoi les raisonnements conjecturaux (l'induction et l'abduction) et les logiques non standard (intuitionnistes et modales, monotones et floues) ne sont pas arrivés jusqu'ici à élaborer des critères, des expressions bien formées afin de distinguer le vraie du faux, la vérité d'une proposition de la vérité d'une modalité? La réponse est simple. Parce que toutes les sciences humaines utilisent la langue naturelle, ambiguë en soi; elles ne sont pas soumises à des règles formelles, à des

\footnotetext{
${ }^{4}$ Jean-Blaise Grize m’a fait observer à ce propos (lettre du 27 mars 2004): «Vous accusez les sciences socio-historiques de s'en tenir au principe de raison suffisante. Leibniz le formulait sous la forme suivante: 'jamais rien n'arrive, sans qu'il y ait une cause ou du moins sans raison déterminante, c'est-à-dire sans quelque chose qui puisse servir à rendre raison a priori, pourquoy cela est existant, et pourquoy cela est ainsi plustot que toute autre façon' (Théodicicée): 'arriver', 'exister', 'être ainsi', bref ce que nous vivons. Une cause de tout cela, une cause qui est dans le temps et pas seulement une raison, qui jamais qu'un pis aller. Et puis il y a le vrai qui ne se réduit pas au non-faux, le vrai qui n'est pas celui d'un modèle, mais celui de la créditivité. [...] Je pense que les sciences sociales, donc humaines, ont pour tâche principale de chercher ce qui a trait à la certitude bien plus qu'à la vérité. Ce qui importe c'est ce qui me prouve quelque chose, ce qui $m$ 'explique quelque chose.[...] Des sciences molles, pourquoi pas des sciences vivantes, des sciences en devenir?».
} 
développements contraignants; elles se servent d'axiomes pris dans des corpus mal définis, approximatifs, la plupart du temps implicites. Même les modèles, fondés sur des hypothèses descriptives et sur des mesures sans théories, ne produisent pas des implications dérivables des prémisses primitives. L'irréalisme des hypothèses initiales favorise, en conséquence, les interprétations normatives des données au détriment de celles descriptives. L'historique de la notion de capitalisme en est l'exemple le plus éloquent. Même la science économique vise moins à décrire et à expliquer qu'à prescrire des orientations et à préconiser des solutions en matière d'allocation des ressources et de leur utilisation. Elle privilégie le raisonnement hypothético-déductif. Pour bâtir ses preuves elle utilise l'histoire économique, la statistique et des modèles mathématiques. Or, cette discipline dépend peu du passé. La statistique n'arrive pas à rendre compte de la mutuelle dépendance des phénomènes ni de la simultanéité des variables. L'économie est surtout déterminée par l'avenir, par les anticipations, les attentes, les aspirations que font les agents sur cet avenir. Les théories des anticipations adaptatives ou des anticipations rationnelles sont irréalistes. Pour cela les théories et les modèles, dans la science économique, ont une fonction surtout prescriptive, normative, jamais une fonction véritablement cognitive.

En sociologie la situation est à la fois plus compliquée et paradoxale. Cette discipline a pour ambition d'interpréter l'expérience sociale des humains et de donner une cohérence à l'ensemble de ces interprétations. Elle prétend à décrypter les matériaux qui constituent la base des représentations informées que les sociétés se forgent d'elles-mêmes et sur elles-mêmes. En même temps, elle revendique le droit de fournir une interprétation serrée du sens de l'expérience sociale des hommes encore que les théories utilisées aient des logique internes propres, que le monde social dans lequel nous vivons change sans répit, que les relations entre l'expérience et la théorie restent abscons et que la vérification d'une théorie par l'expérience est irréalisable. La connaissance sociologique est fondée sur l'irréductibilité des faits sociaux aux faits psychologiques et pourtant elle attribue des pouvoirs causaux aux phénomènes sociaux dépendants des perceptions, des croyances, des préférences, des interactions, des définitions que nous en donnons, des descriptions réalisées avec nos formations discursives. La visée pratique prioritaire de ces formes de connaissances consiste à formater une réalité commune à des collectifs sociaux donnés. Le sociologue formate ces connaissances, les rend intelligibles en les réduisant à unité grâce à des catégories et à des types idéaux. Puis il les valide grâce à l'observation empirique et à la collecte des statistiques. La validation des «faits» est obtenue au prix d'une délimitation des interrelations. Les constructions élaborées de telles manières, dans un contexte donné, par des agents donnés, en vue d'objectifs donnés, ne sont pas des preuves. Elles sont plutôt des activités sociales empathiques car celui qui connaît s'identifie nécessairement à ce qu'il connaît. Il s'agit, certes, de connaissances utiles, mais pas des connaissances vraies puisque leurs démonstrations ne possèdent pas les propriétés des énoncés scientifiques. En sociologie, les énoncés socialement utiles peuvent ne pas être vraies.

Pour illustrer ces remarques rapportons-nous brièvement à L'éthique protestante et l'esprit du capitalisme où Max Weber définit l'esprit du capitalisme en tant que recherche rationnelle et systématique du profit par l'exercice d'une profession et non par l'accumulation du capital et l'éthique protestante comme 
l'accomplissement dans le monde des «devoirs correspondant à la place que l'existence assigne à l'individu dans la société, devoirs qui deviennent ainsi sa 'vocation'». Bien qu'il dise nettement n'exister aucun «rapport immédiat de causalité et de fonction directe entre une doctrine religieuse et un comportement économique », néanmoins ses écrits laissent entrevoir une certaine corrélation, les célèbres «affinités électives », entre l'éthique protestante et les comportements, les mentalités, les usages habituels des capitalistes. Cette corrélation, historiquement invérifiable, ne peut pas être généralisée. Corréler des phénomènes insuffisamment définis, isoler dans une série finie, d'une localité déterminée, pendant une courte période, un facteur particulier, puis en évaluer le degré de corrélation ou de covariance avec le processus général dont il fait partie, tout cela ne peut que produire des artefacts, jamais des preuves consistantes. Weber, certes, en est conscient, puisqu'il rappelle, à plusieurs reprises, que les rapports de causalité n'expliquent qu'une portion du réel, que la science du passé intégral et de la société global est irréalisable. Weber reste, certes, fidèle à l'idée que les preuves sont à la base de l'activité scientifique. Il continue néanmoins à répéter que les phénomènes observables sont toujours donnés - à l'observation intuitive comme à l'observation scientifique - dans le temps et l'espace, et donc dans le cadre de leurs variations spatiales et temporelles.

Cette thèse définit, en effet, une unité logique du cadre cognitif et, en particulier, que les «faits » observables sont indissociables de leur historicité, c'est-à-dire de leurs variations historiques. De cela dérive la nécessité pour la méthode comparative de recourir à différentes espèces d'artifices pour construire des preuves. L'administration de la preuve a également une grande pluralité des formes dans les diverses sciences de l'homme et de la société. L'historicité des faits entre lesquels le raisonnement institue des liens explicatifs n'a pas la même historicité lorsqu'il peut s'appuyer sur un savoir nomologique déjà constitué et lorsque ce même raisonnement ne dispose d'un tel corpus de référence au service de l'interprétation des singularités historiques étant donné qu'il n'a jamais pu conduire ses observations ni mener ses expériences selon la clause ceteris paribus.

Cette «carence nomologique» des sciences sociales n'est ni fortuite ni momentanée; elle découle du type de récurrence ou de transformation des phénomènes historiques, qui se présentent toujours comme des «constellations globales » constituées d'éléments étroitement associés dans une totalité et dotés chacun d'un sens se référant à cette totalité. De telles constellations ne peuvent jamais être complètement décomposées ou analysées en variables et encore moins en variables quantifiables ou en démonstrations.

Grâce à cette distinction, Weber fait une nette séparation entre les sciences intégralement historiques et les sciences de la nature qui restent, en leur fond, des sciences nomologiques, même lorsque ces dernières appliquent leurs connaissances nomologiques à la reconstitution d'une histoire. La distinction entre ces deux régimes scientifiques de la preuve est une distinction entre deux régimes épistémologiques différents d'administration de la preuve historique. L'histoire narrée par une science n'est pas la même histoire selon que peuvent y entrer les « faits virtuels » d'un paradigme théorique unifié ou qu'il s'agit de l'histoire d'une succession ou d'une cohabitation de constellations globales, jamais complètement analysables en systèmes de variations concomitantes entre leurs éléments. 
Ces quelques remarques devraient indiquer que l'idéaltype sociologique (ainsi que la causalité historique) n'est pas un récit historique, c'est une abstraction généralisante, une construction intellectuelle, une utopie, fondée sur des relations abstraites, obtenues en accentuant par la pensée des éléments de la réalité, que nous ne rencontrons jamais dans l'empirie. L'idéaltype vise à former des ensembles cohérents et rationnels à la place des conduites humaines incohérentes et chaotiques. Il est un outil, un instrument de recherche pour rendre intelligible les conduites logiques et non logiques, les expériences vécues des individus, pour comprendre comment elles s'articulent avec les caractéristiques fondamentales d'une société historique donnée.

Weber ne fait pas l'histoire des origines du capitalisme. Il ne fournit aucune preuve sur les origines religieuses du capitalisme. Il essaie simplement de comprendre comment ce phénomène aurait pu être engendré. Il sait que l'histoire traite des consécutions singulières réelles d'une société et que la sociologie ne peut pas les rattacher aux «lois de l'histoire». Toutefois la comparaison du cours «idéal typique» de l'histoire et de son cours réel aide à élaborer des analyses compréhensives dont la valeur est purement hypothétique. Weber met en évidence une affinité particulière, sur le plan des significations, entre certains éléments de la doctrine calviniste et une attitude inédite à l'endroit du monde humain et naturel. A aucun moment Weber a présenté la formation du capitalisme comme un processus déductible de l'évolution des croyances religieuses. Il a voulu jeter quelque lumière sur un phénomène mystérieux, il a continuellement répété que les faits sociaux du sociologue restent foncièrement indéterminés d'où le statut de simple probabilité qu'a la causalité dans la recherche sociologique. Les sociologues d'aujourd'hui savent que la nature de la preuve idéale typique est fort différente de celle prônée par l'empirisme logique et par les sciences expérimentales ${ }^{5}$.

Le dernier exemple je vais l'emprunter à l'histoire. Dans cette discipline, les études sur la notion de preuve ne sont pas nombreuses. Est-ce que la preuve est inadaptée à une activité intellectuelle qui élabore des récits, des «evidentia in narratione», des scénarii crédibles, des représentations de personnages et des situations, des enchaînements plausibles de «ce qu'a été »? En tout cas, le seul à traiter systématiquement de cette problématique est Carlo Ginzburg, chercheur ingénieux, à l'érudition étourdissante, écrivain concis et élégant, critique judicieux du positivisme, du scepticisme et du post-modernisme ${ }^{6}$. Tous ses livres affichent une théâtralité surprenante et sont le résultat d'un montage sophistiqué d'une théorie juste ébauchée, de maintes lectures croisées, de rapprochements inattendus, de digressions profitables. Pour Ginzburg l'histoire s'est constituée au croisement de la médecine, du droit et de la rhétorique. Elle examine des cas et des

5 Pour d'autres développements cf. G. Busino, Encore sur l'éthique protestante et l' esprit du capitalisme, «Bibliothèque d'Humanisme et Renaissance», LXVI, 2004, n. 1, pp. 151-158.

- Pour plus de détails voir ce que j'ai écrit in Pour une lecture des travaux de Carlo Ginzburg. Causalisme, Symétrie et réflexivité, in G. Berthoud et G. Busino, Paroles reçues. Du bon usage des sciences sociales, Genève, Droz, 2000, pp. 399-419. Voir également K. Luria \& R. Gandolfo, Carlo Ginzburg. An Interview, «Radical History Review», 35, 1986, pp. 89-111; Geschichte und Geschichten. Über Archive, Marlene Dietrich und die Lust an der Geschichte. Carlo Ginzburg im Gespräch mit Adriano Sofri, in C. Ginzburg, Spurensicherungen. Über verbogene Geschichte, Kunst und soziales Gedächtnis, Berlin, 1983, pp. 7-24. 
situations dans le but d'en trouver les causes naturelles et de les exposer ensuite selon les règles de la rhétorique. La raison d'être du métier d'historien est la quête des preuves pour s'approcher de la vérité. Mais qu'est-ce qu'une preuve selon cet historien au talent multiforme? ${ }^{7}$

Ginzburg fonde la preuve sur ce qu'il dénomme le «paradigme indiciaire», dont il trouve les bourgeons dans la graphologie, dans la psychopathologie de la vie quotidienne de Freud, dans les travaux du collectionneur Giovanni Morelli, alias Ivan Lermonlieff, auteur des Kunstkritische Studien über italienische Malerei, volume paru à Leipzig en 1893. Le paradigme indiciaire tient compte également des acquis de la sémiotique, de la sémiologie médicale et de l'art des chasseurs d'inférer le passage des animaux à partir de traces observées. Ginziburg est convaincu que si les causes ne sont pas reproductibles, il faut les inférer au moyen des procédures cognitives, grâce aux formes de connaissance sensitives/intuitives susceptibles de dégager et de clarifier les aspects non intentionnels, non contrôlés consciemment de ou des acteurs historiques. Les indices, les traces, les effets, permettent d'inférer les causes supposées. Cette approche, selon Ginzburg, serait étrangère à la logique classique; le paradigme indiciaire serait, par conséquent, différent de celui qu'il dénomme le «paradigme galiléen ». Une telle affirmation est erronée, sans fondement. Dans le «paradigme galiléen » les causes, si elles ne sont pas reproductibles, sont inférées à partir des effets. En plus, le «paradigme indiciaire» n'est pas une nouveauté, il est une formulation revisitée et reformulé de celui de Wilhelm Windelband, le philosophe allemand auquel nous devons la distinction des sciences en nomothétiques et idéographiques. Il faut ajouter également que les graphologues, les chasseurs, Freud, Morelli et tutti quanti, opèrent sur la base d'hypothèses théoriques réflexives ou analytiques présupposant des vérifications, des pointages, des récolements. Le chasseur qui observe une empreinte possède déjà une idée de la forme des pieds des rennes, des bisons ou des élans et des animaux qu'il traque. Le médecin, lui, dispose d'une théorie générale des maladies, Morelli d'une connaissance des tableaux anatomiques et comparatifs des oreilles, Freud de topiques, de traces mnésiques, des doctrines psychologiques sur l'oubli, sur les lapsus, sur les actes manqués et ainsi de suite. La notion d'indice est trop évanescente. Jean-Philippe Dolt, avec son histoire de l'évolution des indices dans les procédures criminelles en France, en Angleterre et en Allemagne, du monde romain à la fin du XVIII ${ }^{\mathrm{e}}$ siècle, a démontré qu'un indice pour être tel doit indiquer les traces de dépendance par rapport au contexte, que cette indication est indispensable pour considérer probables ou valides les conclusions qui se détachent puis de cette dépendance.

Dans les disciplines de connaissance indirecte on doit, certes, procéder par rapprochements d'indices apparemment hétérogènes, mais il faut aussi faire le partage entre les véritables homologies et les simples ressemblances, entre les parentés démontrables et les effets de sens induits par des juxtapositions arbitraires. Si pour décrire un phénomène il faut identifier une séquence plus ou moins

Voir à ce propos F. Pelella, Morfologia e storia. Note sur metodo di Carlo Ginziburg, «Storiografia», 5, 2001, pp. 141-167 ainsi que H. Rebello Cardos Jr., Historians and Detectives: Holmes.Ginzburg. Conjectural Semiotic and Dupin-Veyne's Serial Method, "Storia della storiografia », 44, 2003, pp. 3-20. 
cohérente, alors dans ces disciplines les conditions narratives président à tout acte de compréhension, qu'il soit historique ou bien perceptif. Mais le modèle explicatif narratif n'est pas une preuve.

Dans les livres de Ginzburg, notamment dans Le fromage et les vers, dans Le sabbat des sorciers, dans $A$ distance, les faits réels se mélangent avec les faits imaginaires induits par les pratiques symboliques inlassablement interrogées sous l'angle du décalage et des déformations que le langage peut faire subir au réel. Pour leur auteur la discipline de la preuve en histoire, mais aussi dans les autres sciences conjecturales, est proche du modèle de la procédure judiciaire. Et il croit d'en avoir donné la démonstration avec les essais réunis dans Rapports de force. Histoire, rhétorique, preuve et dans Le juge et l'historien.

Dans ces livres l'auteur dit et répète que la notion de preuve et la quête de la vérité sont à la base du métier de l'historien et de celui du juge. Mais peut-on dire qu'entre les deux existe une certaine proximité alors qu'ils utilisent des méthodes différentes et visent des objectifs divergents? A la vérité, l'historien doit adapter le jugement aux données constatées au moyen de témoignages lointains tandis que le juge doit prendre une décision, la déclarer après un examen des faits, de témoignages proches, et puis la cristalliser en un jugement, la sentence. Pour le juge les faits existent pour autant qu'ils sont nommés par la loi et qu'ils reçoivent d'elle une signification, ils viennent toujours après le droit. La temporalité n'est pas la même en histoire et en droit. En histoire il n'y a pas de prescription, mais uniquement des anachronismes. La logique du droit est binaire (vrai/faux, coupable/non coupable, etc.) et le jugement élaboré est un jugement de réalité, presque jamais un jugement de valeur. Les marges d'incertitudes introduisent les doutes et imposent au juge certaines précautions alors que pour l'historien elles sont des occasions pour approfondir les questions ou les tenir en suspens. L'historien et le juge prennent en compte le contexte, mais pas de la même manière. Pour le juriste le contexte fournit une circonstance qui vient de l'extérieur atténuant ou aggravant une responsabilité, éclairant ou pas la logique d'une action. Pour l'historien il donne à l'action tout son sens et il se substitue à elle. Le contexte (social, politique, culturel, psychologique) est le «lieu de possibilités historiquement déterminées, sert à combler ce que les documents ne nous disent pas sur la vie d'une personne. Mais il s'agit là d'éventualités, pas de conséquences nécessaires; de conjectures, pas de faits avérés ». Ginzburg sait qu'il n'est pas toujours possible d'obtenir une preuve, c'est-à-dire «la possibilité de prouver que x a fait y; $\mathrm{x}$ pouvant désigner indifféremment le protagoniste, éventuellement anonyme, d'un événement historique ou le sujet impliqué dans une procédure pénale». Il est pourtant convaincu que le travail de l'historien, bien qu'il ne puisse pas faire de l'expérimentation vu que les phénomènes étudiés sont irréversibles, arrive néanmoins à produire des résultats dans l'ordre des probabilités de la preuve.

Ces thèses soulèvent des nombreuses interrogations et beaucoup de doutes. On le constate en parcourant, par exemple, la littérature sur le "génocide des Arméniens », sur la Shoa; on l'a constaté de très prés lors du procès Papon, à l'origine d'innombrables controverses entre juristes, historiens et philosophes.

Les différences entre l'historien et le juge dérivent non seulement des diversités des traditions intellectuelles mais surtout de la différence des approches et des solutions données aux problèmes. Le juge doit résoudre, d'après une qaestio juris, une quaestio facti, qui lui a été soumise par une ou des parties (nemo iudex 
sine auctore). Son activité, gouvernée par les lois positives, se borne à l'examen des faits qui lui ont été soumis. L'historien, par contre, choisit son sujet, formule ses questions et utilise, à sa guise, tous les matériaux qu'il juge utiles pour y répondre. La preuve judiciaire a pour fonction de faire naître une conviction sociale alors que la preuve scientifique vise à établir une vérité, provisoire, plus ou moins détachée de l'utilité sociale. Les propositions découlant d'une présomption sont retenues, par le juge, comme descriptives du cours normal des choses tandis que l'imputation d'un risque devient une méthode pour résoudre des litiges. L'exemple du principe de précaution est éloquent à ce propos. La preuve juridique vise à légitimer les décisions de l'autorité et des institutions, à structurer des représentations comme celles d'adhésion, de faute ou encore di négligence. La décision judiciaire s'impose super partes; elle est la solution donnée à la controverse. Ce n'est jamais le cas en historiographie. Alors que le juge doit donner la solution aux questions qu'on lui ont été posée, l'historien peut suspendre son jugement et laisser à d'autres temps la charge des questionnements, à d'autres historiens la quête d'autres archives. Alors qu'il n'y a pas de problèmes historiques définitivement classés, la sentence du juge devient définitive à un certain moment et la prescription entraîne l'extinction du problème. Les historiens, à chaque génération, recommencent à écrire différemment les mêmes histoires. L'historien n'est pas un juge; le juge n'est pas un historien. La preuve du juge n'est pas celle de l'historien. Il en est de même également de la quête de la vérité.

Il est temps de conclure. Le régime de la preuve dans les sciences de l'homme et de la société est multiforme, incertain, indirecte, conjectural. Le chercheur doit confronter des témoignages, il ne peut pas donner aux découpages un commun dénominateur. En l'absence de différentes versions il est obligé de prendre en charge des faits sans pouvoir les recouper. Ceci a fait écrire à Popper dans Misère de l'historicisme que les disciplines socio-historiques produisent uniquement des bavardages et des verbiages. La réprobation est injuste. Les sciences de l'homme et de la société possèdent un régime spécifique de scientificité; elles sont dotées d'un appareillage conceptuel, plus ou moins stable et cohérent, ayant une capacité auto organisatrice relativement solide. Elles ont en commun avec les sciences exactes quelques problématiques, par exemple le rapport sujet/objet, la complexité du réel, la relativité des points de vue, la relation avec le sens commun, les structures non observables, les phénomènes kaléidoscopiques et bien d'autres choses. Les sciences sociales différent et se séparent des sciences dites «dures » à cause de la nature et de la spécificité de nos investigations. Celles-ci portent invariablement sur des événements en situations singulières, irréductibles, sur des phénomènes irréversibles, indécomposables, insubstituables, phénomènes que nous appréhendons à travers des contextes particuliers et des coordonnées spatiotemporelles incommensurables. Dans nos sciences sociales, il est impossible de comparer le singulier et d'analyser des coordonnées incommensurables. Dès lors il ne reste qu'à recourir à des paris interprétatifs, élaborer, en langage naturel, des généralisations d'une espèce particulière, ni hypothèses ni interprétations analysables statistiquement, testables expérimentalement. Tous les énoncés, de nature foncièrement conjecturale, sont toutefois plausibles car basés sur des représentations sociales, sur les savoirs communs à une collectivité, sur des dates et des documents en partie vérifiables et sur des interprétations indirectement vérifiables et d'ordinaire plausibles. En postulant que les acteurs sociaux, que les agents 
historiques savent ce qu'ils font et interprètent leurs propres actions, le chercheur peut alors construire son savoir et ses interprétations sur la base de ces savoirs et des ces interprétations-là.

Les sciences sociales ont conscience de pratiquer une pluralité de constructions théoriques, d'approches différentes, d'utiliser des multiples cadres d'analyse, d'élaborer des preuves relativement indépendantes des théories en tant que systèmes conceptuels ou ensembles propositionnels. Elles savent que le pluralisme théorique engendre un pluralisme des faits, que l'accès aux preuves empiriques est ardu, que l'utilisation de la langue naturelle favorise peu l'épuration des constructions théoriques et non théoriques, que la cumulativité est inexécutable et l'existence de paradigmes stables chimérique. Elles savent, en outre, d'être des représentations des représentations des pratiques historiques, que leurs concepts, leurs notions, leurs constructions transforment les matériaux qu'elles étudient, que les connaissances ainsi produites sont sans cesse réappropriées par les acteurs et par les institutions.

On peut expliquer ce pluralisme par la dimension historique de toutes les sciences sociales, par le fait qu'elles doivent se servir des langues naturelles et peu des langages formels, que l'intelligibilité des systèmes sociaux, toujours en devenir, est conditionnée par les manières de les représenter, de les dire. Jean Blaise Grize appelle cette spécificité des sciences socio-historiques, leurs opérations de réduction à l'essentiel, «la schématisation ${ }^{8}$. En effet, nous schématisons l'information lorsque nous construisons un ensemble signifiant, dans le sens que ce qui est dit reste inséparable de sa mise en forme. La «schématisation», selon Grize, révèle comment le discours appréhende la situation dans laquelle il se produit, la manière selon laquelle il est structuré en fonction d'une perspective donnée, de même que les réglages effectués en cours de construction et de transformation. Ce processus de schématisation, avec le cortège des opérations de détermination, comme la constitution d'objet, la prédication, la mise en relation, et en outre des opérations d'acceptabilité, de cohésion, ce processus est inhérent à toutes les opérations des sciences socio-historiques. Comme toute argumentation, les théories sociales s'adressent à un auditoire. Elles impliquent, par conséquent, des interventions acceptables, des opérations d'éclairage plus ou moins en rapport avec des valeurs, des idéologies, avec les standards des connaissances en cours dans la communauté scientifique, avec les sagesses du système culturel d'appartenance. Les savoirs sociaux présupposent un consensus, des compétences liés aux situations dans lesquelles ils se produisent et des pertinences en fonction d'une perspective finalisée. Dans cette logique argumentative, il n'y a pas de place pour une théorie unifiée et englobante offrant de solutions aux problèmes sociaux et à ceux de l'organisation sociale. La raison est évidente: les questions sociales changent ou resurgissent au gré du hasard des schématisations, des points de vue et des éclairages différents. Pour cette raison, les concepts et les notions des sciences sociales sont la plupart du temps métaphoriques; pour cette raison les disciplines sociales doivent faire des emprunts à d'autres disciplines et utiliser analogiquement les catégories d'identification et de référenciation.

s Voir ce que j'ai écrit in Lecture de Grize, in G. Berthoud et G. Busino, Paroles reçues. Du bon usage des sciences sociales, cit., pp. 355-367. 
Dans les sciences socio-historiques il n'y a jamais eu, il n'y a pas de correspondance biunivoque entre les faits et les propositions théoriques. Les chercheurs y formulent des propositions non équivalentes selon les régions et les époques, selon les situations et les conditions particulières et spécifiques. Dès lors il est pratiquement impossible de falsifier une théorie sociale, par exemple la théorie du suicide de Durkheim, celle de la bureaucratie de Max Weber, celle de la circulation des élites de Pareto ou la dynamique du capitalisme et la grammaire des civilisations de Braudel. Il est courant, par contre, que des théories formellement fausses soient significativement signifiantes et manifestent des apparences d'application. La vérité d'une théorie sociale n'est guère vérifiable, tandis que son utilité sociale, au demeurant variable, peut être facilement observée.

Liées aux activités pratico cognitives des hommes et des groupes sociaux, guidées par la raison délibérative et animées par la pensée argumentative, nos théories sociales, changeantes et provisoires, engendrent des «faits» mais ne correspondent jamais à des «faits», ne sont jamais validées que par des «faits» produits par elles-mêmes. Les sciences sociales sont prisonnières d'un univers sémantique dont la clôture reste insurmontable.

Comment faire correspondre une hypothèse aux faits alors que nous sommes dans l'impossibilité absolue de préciser préalablement quand telle ou telle autre correspondance aura lieu ou bien quand nous nous approcherons de l'une d'elles? Il s'agit là d'une impossibilité absolue puisque la conjecture au sujet de ces faits a la même signification que l'hypothèse qui parle de ces mêmes faits. En plus, le langage baigne dans de la signification et c'est précisément pour cette raison que nous comprenons ce qu'on nous dit pas du tout à partir des règles formelles du dit langage.

Les sciences sociales sciences de l'interprétation du sens des comportements humains, sont aussi celles des interprétations du sens des autres langages; elles ne peuvent pas être réduites aux sciences de l'expertise et de l'application car totalement dépourvues des moyens nécessaires pour réaliser des tâches pratico-politiques. Par contre, les sciences sociales ont une tâche plus essentielle, voire absolument incontournable: donner du sens aux rapports sociaux, rendre intelligibles les rapports gouvernés par des logiques brutes ou naturelles, par les logiques des sentiments; garantir qu'aucun corps constitué de savants et de techniciens ne pourra jamais prendre le pouvoir en s'appuyant sur la force ésotérique de son langage. En reconnaissant et en valorisant les différents modes de rationalité, en distinguant la rationalité de l'action de la rationalité de l'explication, en acceptant l'existence de au moins deux formes de logique, celle de la démonstration et celle de l'argumentation, en admettant que la vérité ne coïncide pas avec l'utilité, qu'une doctrine non vraie peut être socialement utile alors qu'une doctrine vraie peut être socialement nocive, les sciences sociales sont, sans aucun doute, la science et la conscience de la modernité.

\section{LE DROIT ET LA JURISPRUDENCE, SCIENCE STORICOSOCIALE?}

1. Les transmutations, puis le déclin du paradigme juridique centré sur la loi sont bien connus. Les pièces maîtresses de ce paradigme (généralité, abstraction, 
répétitivité, reproductibilité) étaient vissées entre elles par les logiques de l'identité et de la réversibilité et assurées par une raison indifférente aux spécificités et aux particularités des sujets individuels, aux singularités des contextes sociohistoriques, faisant l'économie du concret et de l'individuel, principalement soucieuse de déterminer les rapports juridiques entre des sujets de droit.

Pendant longtemps, on a cru que la réalité, physique et sociale était soumise à des principes essentiels, constants, nécessaires, en bref qu'elle était gouvernée par des lois. Certes, la notion de loi a beaucoup variée au cours du temps (la loi comme raison, la loi comme uniformité, la loi comme convention, loi comme relation symbolique); certes, les propositions énonciatives formulant un rapport constant entre les faits étaient des formules pour la prévision, réduisant la validité à l'efficacité, aux conditions d'obtention de prévisions plus ou moins exactes. L'explication devait ramener toujours un fait ou un événement à une loi. Pour appliquer la logique de la reproductibilité identitaire il fallait recourir à des procédures d'homogénéisation et d'égalisation.

Les actions humaines on pouvait alors les expliquer par une loi, celle fixée par la norme, impérative, obligatoire, bien que sans nécessité absolue. Expliquer et juger le pourquoi et le comment de tel ou tel autre comportement, le qualifier de légitime ou d'illégitime, de licite ou d'illicite, découlait nécessairement de la norme élevée au rang de variable indépendante.

Aussi longtemps que nous avions à faire avec des «objets» stables et reproductibles, avec la loi source de tous les systèmes normatifs, la compréhension et la gestion du monde sociale pouvaient être prises en charge par les modèles de rationalité fondés sur la déduction et sur l'induction. Cette situation a été bouleversée dans les sociétés du capitalisme industriel, intensément conflictuelles, en continuelles et rapides transformations, nécessitant, en plus du contrôle social ordinaire, de normes de direction et de régulation de différentes espèces démarquant mal les sources matérielles et les sources formelles et modifiant aussi les fonctions mêmes du système juridique.

Friedrich von Hayek a analysé cette transformation et en a placé l'origine dans le Welfare State, dans l'Etat providence, dans l'Etat social, dans l'Etat fiscal. Selon cet économiste néolibéral, dans l'Etat libéral classique, il y avait uniquement des normes primaires, ou normes de conduite, alors que dans l'Etat social il $\mathrm{y}$ a une forte augmentation des normes secondaires, ou normes d'organisation. Bien que les normes d'organisation soient fondamentalement des normes de conduite, Hayek postule qu'en vertu de ces normes, l'Etat social doit faire faire et doit faire lui-même. Puisque les normes d'organisation règlent également l'action de tous les organes de l'Etat social, la prépondérance est absolue, dans les sociétés contemporaines, du droit administratif et du droit public en général sur le droit privé constitutif auparavant des fondements mêmes des sociétés libérales d'antan.

Dans l'Etat libéral classique la loi était tutélaire et garante; dans l'Etat postlibéral, elle est promotionnelle, stimulatrice, incitatrice. De générale et abstraite, préétablie et impérative, la loi est devenue singulière, concrète, ponctuelle. Contrairement aux lois sanctions, les lois de l'Etat social visent à produire, moyennant directives, subventions, crédits facilités, exonérations d'impôts, exceptions, etc., des résultats programmés.

Bien que l'analyse de Friedrich von Hayek soit biaisée par des prénotions insatisfaisantes, elle met pourtant en lumière une singularité de nos sociétés 
contemporaines où il n'est pas aisé de rapporter, sic et simpliciter, les relations et les interactions de la vie sociale à une loi ou à une règle de droit. Ces sociétés, en raison des innovations techniques et économiques qu'elles favorisent, évoluent sans cesse selon de dynamismes internes indiscernables, aux effets cumulatifs, parfois surprenants. Les normes juridiques, en cristallisant la vie sociale en devenir constant dans des règles trop strictes, deviennent elles-mêmes des sources de conflits. Les transformations des sociétés contemporaines, au consensus social émietté, toujours prêtes aux révoltes ou aux explosions de violence, s'accommodent à peine avec le formalisme de l'ordre juridique davantage rigide que flexible.

Dans ces sociétés le droit n'est plus l'unique garant de l'équilibre social ni le seul régulateur des rapports sociaux. Il est en concurrence avec d'autres systèmes normatifs, économiques, scientifiques, religieux, pédagogiques ou autres, qui fixent des règles, qui défendent leurs autonomies, qui essaient d'échapper à toute tentation de hiérarchisation, de subordination. Pour les systèmes normatifs non juridiques, le droit n'a qu'une validité statutaire. Le modèle juridique est fait de normes générales, lesquelles ne prennent pas en compte les particularités et les spécificités des situations et des contextes, pas plus qu'elles ne peuvent établir si une véritable situation d'exception est donnée.

Pour fournir des mécanismes de solution aux activités sociales, économiques, politiques ou religieuses, pour surmonter les conflits qui naissent dans les sphères spécifiques, la loi, selon certains critiques, devrait renoncer à ses propres principes fondateurs, c'est-à-dire à la généralité, à l'abstraction, à la répétitivité, à la reproductibilité. Dans les sociétés conflictuelles où le pouvoir et l'obéissance subissent des ébranlements permanents, la loi n'est plus conçue et perçue en tant qu'expression de la volonté générale du corps social. Elle y est rabaissée au rang d'une simple mesure réglementaire ou de procédé pour communiquer la volonté gouvernementale sur une question donnée. Dans ces sociétés la pléthore de lois, que le citoyen est dans l'impossibilité de connaître, porte sur des problèmes particuliers qu'on entend orienter ou contrôler. Les difficultés de l'interprète pour les ajuster à la variété des conflits et aux cas concrets sont considérables et soulèvent des questions essentielles et très compliquées. En effet, les normes générales et abstraites s'appliquent mal aux situations concrètes, d'où la création d'inégalités entre les égaux et d'égalités entre les inégaux. Les anciens critères pour qualifier les égaux, pour régler, traiter, administrer les rapports sociaux, pour déterminer la légalité et la causalité, sont devenu pour ainsi dire obsolètes.

On dit que nos sociétés sont ingouvernables car conflictuelles, conflictuelles car les modèles de loi les régissant sont obsolescents, et ils sont tels car le pouvoir politique a subi des transformations substantielles. Les sources normatives traditionnelles doivent désormais coexister avec des sources extra-législatives, voire même extra-étatiques. Le monopole de la production juridique par le pouvoir législatif représentatif est ébranlé. En conséquence, l'activité créatrice des juristes en général et du juge en particulier est au plus haut point stimulée. Très attentif aux phénomènes sociaux, le juge est porté à suivre de près toutes les mutations sociales. L'image du juriste conservateur des règles données, dépositaire et gardien des lois, chargé d'interpréter le droit et de l'appliquer, laisse la place à celle du juge appréciant les faits dont les normes juridiques constituent des évaluations, s'efforçant de trouver les règles adéquates et idoines, appropriées afin de résoudre des problèmes compliqués et aux implications sociales ou 
économiques complexes. Que le juge soit en train de modifier le blason de la légalité classique (gouverner ou juger), qu'il soit en train de devenir producteur actif de droit c'est un fait de plus en plus évident. Désormais, il cherche les modèles normatifs tant dans les règles du système juridique établi que dans les rapports et valeurs sociaux. Le droit de science autonome et pure devient peu à peu une branche de la science générale de la société.

L'autre transformation importante est celle concernant les fonctions des modèles normatifs juridiques. Les fonctions répressives, basées sur les sanctions, font une place notable aux fonctions promotionnelles, aux normes techniques visant la prévision et l'anticipation. Le contrôle persuasif assuré par le conditionnement psychologique accompagne le contrôle contraignant. La réaction sociale précède le comportement ou l'événement et l'intervention-remède remplace l'intervention-prémonition. La prévention ancienne était de nature intimidatrice, la nouvelle utilise les sciences psychosociologiques pour empêcher l'élaboration et l'émergence des comportements non souhaités, pour gérer des situations singulières, concrètes, pour contrôler la production, la distribution et la consommation de biens rares et de ressources périssables.

Est-ce qu'une société où toute forme de déviance est effacée avant même son surgissement, une société décourageant systématiquement les comportements déviants au profit des comportements souhaités, est-ce que cette société-là peut encore se nommer société de droit? Quelle est la place du droit comme organisation légitime de la force dans une société où l'ordre juridique fait d'abord de la promotion, en offrant des services, en orientant la distributions des biens, en ne se bornant plus uniquement à protéger les individus et à garantir la circulation des biens et des richesses?

Les marques de cette transformation radicale de l'Etat libéral de droit en Etat social post-libéral sont manifestes: les moyens de contrainte effectivement à disposition sont réduits tandis que les possibilités de désobéissance, de résistance passive aux ordres, de contestation, d'opposition, de dissidence, de refus augmentent. Le droit, dès lors, assure mollement la stabilité et la continuité des rapports sociaux. Et plus la pléthore des lois, des règlements et des directives est grande, plus le chaos normatif sape les fondements du principe de la certitude du droit et en fait un instrument foncièrement politique.

Ce n'est donc pas un hasard si aujourd'hui on commande avec des lois, on administre avec des lois, on soumet la décision politique à des procédures de consultation réglementées. Le droit en tant qu'expérience inquiétante se plaçant entre la certitude de la force et l'incertitude de la justice, ce droit-là périclite doucement et va à sa fin. Faut-il s'en inquiéter, est-ce inéluctable, faut-il en désespérer?

Devenu objet et source de conflits, le droit répond mal aux fonctions qui ont été les siennes auparavant. Les conflits qu'il avait pour tâche de résoudre n'étaient pas généralement juridiques, mais il arrivait à leur conférer un caractère juridique pour les solutionner. Actuellement, il est difficile de fournir les critères juridiques appropriés à la solution des conflits sociaux, à maîtriser les crises selon les méthodes juridiques. Les sociétés contemporaines foncièrement conflictuelles, en mettant en évidence l'impuissance du droit, l'impossibilité de répondre pleinement aux exigences de sa propre finalité, en accélèrent la crise, la détresse, le désarroi. 
Y a-t-il des solutions pour enrayer cette crise qui pourrait se révéler fatale pour les démocraties libérales? Faut-il renoncer à l'identification du droit avec la loi? Faut-il explorer d'autres formes de rationalité, construire d'autres paradigmes? Faut-il prendre acte du fait que les organisations administratives assument un rôle spécifique et relativement autonome dans l'exécution et dans l'application des décisions touchant aux multiples aspects de la vie sociale, de nos activités, tant dans la sphère publique que dans la privée? Est-ce que d'autres modèles de rationalité pourraient mieux adapter les décisions aux situations concrètes, et corriger ainsi les difficultés propres à la généralité, à l'abstraction, à la répétitivité, à la reproductibilité, à cette rationalité définie improprement de galiléenne? Comment peut-on échapper aux méfaits tyranniques, inhumains, injustes de l'application du strict légalisme ou du recours sans contrôle au seul principe de l'efficience ou de la convenance?

Si le droit doit continuer à ordonner la vie en commun des hommes, s'il doit rester l'instance commune aux diverses activités humaines, s'il doit continuer à être le seul moyen pour trouver une solution équitable aux conflits entre les sphères des rapports sociaux, économiques, ou politiques, alors il faut prendre acte du fait que le modèle de la loi, celui de la généralité, de l'abstraction, de la répétitivité, de la reproductibilité, que ce modèle de rationalité est inadapté aux rapports sociaux d'aujourd'hui.

Nos sociétés s'organisent, se désorganisent et se réorganisent sans cesse pour s'adapter aux circonstances, pour établir une certaine équité entre leurs membres, entre les générations, mais aussi pour s'approcher des idéaux qu'elles se donnent à elles-mêmes. Pour cette raison, la loi ne peut plus se réduire à un ensemble de règles codifiées ou codifiables hic et nunc. Désormais les conduites, les actions, les comportements ne peuvent pas être épuisés par une application pure et simple des normes juridiques. En effet, l'efficacité des interactions sociales dépend de la possibilité de transgresser les normes et éventuellement d'en créer d'autres. Les normes sociales dépassent de beaucoup l'ensemble des normes juridiques. $\mathrm{Ni}$ l'ordre social ni l'ordre politique ne sont assimilables à l'ordre juridique en tant qu'un ensemble de conventions et de règles qui orientent les activités humaines. Faut-il continuer à raisonner comme si le droit s'appliquait exclusivement à des situations juridiques? Faudrait-il plutôt reconnaître que le droit s'applique à des situations non juridiques qu'on essaie de ramener à des catégories juridiques pour arriver à une solution moyennant une procédure rigoureuse et préétablie? Le cas échéant, ne faudrait-il pas formaliser mieux le rôle de la médiation, celui de l'arbitre ou du conciliateur?

2. A toutes ces interrogations les juristes contemporains ont essayé de donner des réponses réalistes et plausibles. En effet, dès la seconde moitié du $\mathrm{XX}^{\mathrm{e}}$ siècle une nouvelle topographie tente de configurer autrement le domaine du droit. La philosophie du droit des philosophes centrée sur l'ontologie, sur la gnoséologie et sur l'éthique et fragilisée par la sociologie du droit, va être subvertie par les recherches des juristes. Les travaux de théorie générale, les études de la règle en tant que telle et de sa structure, influencés pendant longtemps par les doctrines de Hans Kelsen, avaient réservé la place la plus importante aux structures normatives, aux énonciations sur des énonciations, à la logique déontique au détriment des fonctions et des situations conflictuelles. La source du droit est la loi, formulée 
de façon générale et abstraite, par un organe désigné par la Constitution. Le droit est le domaine du Sollen, il est un univers non factuel, un monde suprasensible où l'imputation efface la causalité. Les investigations, exprimées en un langage prescriptif, au moyen d'énoncés déontiques et de dichotomies, portent principalement sur les normes, c'est-à-dire sur des entités non factuelles. Le droit est défini par la contrainte, par la norme impérative, par la primauté de la loi, par la complétude et la cohérence de l'ordre juridique, par une vue mécaniste de l'interprétation et par un net rejet du logicisme. Puisque le juste est ce qui est conforme au droit positif, il en découle qu'il faut lui obéir quel qu'il soit son contenu.

Une nouvelle topographie va configurer désormais le domaine du droit. A partir des années '60, le positivisme juridique amalgamant l'éthique étatique à l'approche légaliste et formaliste de la justice, suscite moult critiques, des contestations tant il est vrai que son hégémonie culturelle en sort déstabilisée. L'apparition d'autres sources de droit (extra-législatives et même extra-étatiques) rend chancelant le monopole de la loi sur la production des normes. L'Etat, soumis aux processus d'internationalisation de normes postulant l'existence de valeurs universelles, doit admettre que d'autres acteurs élaborent des règles en fonction d'intérêts sectoriels et particuliers. Une nouvelle philosophie du droit élaborée, cette fois-ci, par des juristes praticiens rénove de fond en comble la problématique traditionnelle et s'ingénie à délinéer les fondements d'une théorie générale $\mathrm{du}$ droit, à caractériser la scientificité de la jurisprudence, à analyser les modes de productions des raisonnements des juristes ainsi qu'à définir le droit en tant que norme, en tant qu'institution et en tant que rapport intersubjectif réciproque. La formation, l'évolution, la structure formelle, les valeurs à la base de l'ordre juridique vont se trouver ainsi au cœur de débats foisonnants et riches d'ouvertures inattendues et prometteuses. Les transmutations dans le temps du droit positif, ses singularités spatiales, révèlent que sa nature est contingente et composée de particularités. Le monisme est abandonné et le pluralisme remet en discussion les vielles doctrines du normativisme, de l'institutionnalisme, du décisionnisme, du structuralisme et du fonctionnalisme. Plutôt que commenter, interpréter, déterminer la signification des règles incompatibles, floues ou lacunaires, de gloser sur les sources matérielles et formelles, on évalue aussi les rapports sociaux et leurs contextes et on recherche, au moyen de techniques différentes, à la fois les moyens pour les régler et les formulations pour les rendre plausibles.

La fonction du juriste va changer. Il n'est plus le dépositaire, le gardien, le transmetteur des règles, le conservateur des modèles normatifs. Désormais il transforme, intègre, donne des significations précises aux règles et cela grâce aussi au recours aux variables institutionnelles, sociales, culturelles, idéologiques. Avec des procédures de typification et d'indexation du sens dans le contexte de l'action, il élabore les critères d'appartenance et de non appartenance, d'inclusion et d'exclusion; il peut fixer même les frontières entre les groupes. En somme, sa jurisprudence est de plus en plus proche de la «verstehende Kulturwissenschaft» théorisée par Gustav Radbruch.

Les juristes spécialistes du droit social sont à l'origine d'une production juridique intense, certes favorisée par les politiques publiques de programmation socio-économique et de planification industrielle, laquelle a assuré à leur discours le pouvoir de faire exister dans la pratique ce qu'ils stipulaient. A son tour, le droit du travail a pris en charge les négociations collectives, les contrats de branches et 
de catégories dont les règles coiffent souvent les dispositifs de la loi. De même, les professionnels du droit de l'économie, du droit commercial, du droit industriel, du droit des entreprises, vont s'efforcer d'ajuster le formalisme de ces disciplines aux transformations sociales en cours dans les sociétés démocratico-libérales à économie de marché. Et c'est ainsi qu'ils ont contribué à rendre le droit plus adhérent aux problèmes de la vie sociale, du développement et des transformations socio-économiques. Même les civilistes, longtemps gardiens de l'autonomie du droit, créateurs de toutes les techniques utilisées communément et pendant longtemps par la communauté des juristes, participent à ce travail de renouvellement et agrandissent notamment des instituts tels que le testament, le mariage, le divorce, la paternité, la filiation,etc. Mais si l'on indexe le droit aux rationalités politiques et sociologiques, si l'on rapproche les formes de connaissance aux modalités du pouvoir, si la réalité sociale est la cause du droit et ce dernier en est sa légitimation, ne risque-t-on de se trouver devant une énorme aporie, voire à une tautologie?

Les travaux juridiques des dernières décennies reconnaissent la spécificité du droit mais ils montrent aussi les failles d'un formalisme indifférent aux contenus, attentif exclusivement aux formes logico-linguistiques à travers lesquelles l'expérience s'exprime. Les faiblesses du raisonnement juridique fondé sur les dichotomies, le flou de la hiérarchie des normes (les primaires qui imposent des obligations et les secondaires qui sont tantôt des normes sur les normes et tantôt des normes qui confèrent le pouvoir), l'irréalisme de la réduction de l'acte de juger à un syllogisme, sont analysés avec la plus grande rigueur et doigté.

Bon nombre d'autres problématiques traditionnelles ont été revisitées sans pour autant être totalement abandonnées. Des questions fondamentales n'ont pas encore trouvé de solutions satisfaisantes, par exemple celles-ci: la jurisprudence est-ce une science formelle ou une science empirique? Est-ce la norme à donner la qualification juridique aux faits sociaux? Est-ce juridique le contenu du fait ou bien sa qualification? Peut-on concilier l'analyse structurelle avec l'analyse fonctionnelle de l'univers juridique? Si pour accéder à l'objet il faut passer par l'interprétation, la science du droit n'est rien d'autre qu'un métalangage descriptif. Dans ce cas le langage objet est le discours prescriptif du législateur. Mais s'il n'y pas une science juridique en dehors des techniques interprétatives comment celles-ci peuvent être neutres et ne pas dépendre des finalités politiques?

Certes, la vérité textuelle est fondamentale dans tous les savoirs sur la société. Cependant lire comme un texte les rapports sociaux qualifiés par le droit peut devenir une sorte de «disposition cérémonielle» du pouvoir interprétatif. Puisque l'exercice de celui-ci requiert toujours des pratiques symboliques qui accompagnent et redoublent les autres sources de reconnaissance (institutionnelles, répressives, participatives et discursives), les dangers d'une perte de l'autonomie et de l'indépendance sont bien réels.

Sur tous ces points les chercheurs en droit administratif ont fait preuve d'audace, de dynamisme et d'un bel esprit entreprenant. L'un d'entre eux, depuis une bonne dizaine d'année, vient de labourer ces problématiques avec minutie, rigueur, une ingéniosité très originale et des trésors de perspicacité. Ces qualités on les retrouve dans le dernier livre de Pierre Moor, véritable petit traité d'épistémologie juridique, intitulé Pour une théorie micropolitique du droit (Paris, PUF), où le professeur de droit administratif de l'Université de Lausanne systé- 
matise et synthétise ses précédents travaux théoriques tous adossés à des cas concrets.

3. Pierre Moor est réputé pour ses avis de droit, pour son traité de droit administratif, pour avoir analysé ou expertisé les questions les plus compliquées et complexes de la science et de la pratique administratives. Depuis deux ou trois lustres, parallèlement à cette activité-là, Moor s'interroge sur la nature du droit administratif, sur l'importance de la juridiction administrative, sur ses spécificités par rapport au droit public en particulier et par rapport à toutes les autres disciplines juridiques, à la justice procédurale et substantielle et à la science sociale et politique en général. Ayant pris la mesure des limites de la logique dans le raisonnement des juristes, il a voulu en découvrir les raisons et élucider comment le savoir juridique se produit, est produit et puis il est reproduit.

En lisant et relisant Moor on acquiert la conviction que son penchant pour le travail théorique est notable: les définitions et l'interprétation du sens des énoncés, la manière de dévoiler les connotations axiologiques des expressions sont à la fois subtiles et élégantes. Pourtant son détachement au regard des grandes passions du monde contemporain ainsi que ses options culturelles restent impénétrables. L'écriture est concise, sobre. La connaissance des auteurs classiques et contemporains est étendue, cependant le choix des œuvres citées est très sélectif, non dépourvu, en vérité, d'une certaine préciosité. La propension à l'aventure intellectuelle rend l'auteur indocile à l'endroit du positivisme juridique, des traditionnelles dichotomies (droit public et droit privé, etc.), de la distinction entre le droit et la science juridique, mais il ne polémique pas et ses réticences ainsi que ses idiosyncrasies restent bien dissimulées.

Contrairement à une tradition bien établie, Moor n'aborde pas la problématique à partir de son traditionnel noyau dure. En effet, il ne commence pas sa recherche épistémologique par la dissertation courante sur la nature et les finalités du droit, sur sa place dans les sociétés industrielles, sur l'autonomie et l'indépendance du système normatif, sur le statut de la théorie du droit, sur les fonctions et les raisonnements des juristes ni même sur le droit en tant que science formelle ou science empirique. Il avoue au préalable que pour lui le droit est «le système» de représentation de la société et qu'il va essayer d'en donner la démonstration pas à pas. En partant de la marge extérieure là où les pilastres en gaine de l'édifice ont été placés par la tradition doctrinale, puis d'échafaudage en échafaudage, un étage après l'autre, Moor arrive au centre (le pouvoir, l'autorité, la politique) de la question et nous la montre dans tous ses aspects kaléidoscopiques.

Essayons d'entrevoir le fil rouge de cette recherche en suivant rapidement l'itinéraire tel qu'il nous a été proposé. Moor postule que le droit est un système de représentations des valeurs sociales et des autorités hiérarchisées qui attribuent et distribuent le sens indispensable à l'exercice du pouvoir. Il est, pour ainsi dire, le conjugateur de la liberté et de la contrainte. Pour saisir le droit en son entier, il faut découvrir son mode de fonctionnement, et plus précisément les acteurs le rendant possible. Dans cette perspective, le droit ne se réduit pas à la règle, ni même à son contenu, à son impérativité, à la contrainte. Sa raison d'être se trouve dans le passage de la norme à la décision. Le travail des acteurs chargés de lier la norme générale abstraite au fait historique, au rapport social concret, effectif, est donc fondamental. Ces acteurs disposent d'un pouvoir dont l'exercice est impré- 
visible, voire subjectif et arbitraire là où il n'est pas discipliné par la loi et/ou là où il découle de notions juridiquement indéterminées, lacunaires, d'énoncés flous ou ambigus.

Pour obtenir la liaison entre la norme générale abstraite et le cas individuel concret, en somme pour appliquer la norme il faut tout d'abord l'interpréter. L'interprétation à l'intérieur du système permet de réduire à l'unité la multiplicité des sens possibles, de «conférer une signification déterminée [dotée de force contraignante] à la situation concrète qu'il faut juger». Mode de production des significations, «Transmutation de la norme en décision» grâce à «un travail de rationalisation portant sur le cas concret à partir de notions figurant dans une proposition formulée en termes abstrait, le droit est une structure politique spécifique, une institution social-historique, à la fois théorie et pratique. Ce n'est pas la norme à le caractériser mais plutôt la norme et son application. Ensemble, elles forment «un système dans lequel le texte normatif ne peut être 'norme' que dans et par son application et l'acte d'autorité ne peut être 'application' que dans et par le texte normatif auquel il se réfère ».

Dans cette perspective le droit est « un système social, composé d'un ensemble de textes normatifs que produit et utilise un ensemble d'acteurs défini par ce système lui-même, aux fins d'émettre des actes dont la société reconnaît la nature impérative » et dont l'objectif est le maîtrise et la gestion des conflits, la pondération et la comparaison des facteurs individuels concrets en jeu.

La légalité (respect de l'ordre public, tout acte doit se fonder sur la loi, traiter les situations semblables de manière semblable et les différentes en différente) et la prééminence de l'intérêt public sur l'intérêt privé opposé, jusqu'ici ont été réputés des principes constitutifs du droit de l'Etat de droit. Moor, après avoir analysés minutieusement ces deux principes, conclut qu'ils dissimulent la dimension micropolitique et son institutionnalisation socio-historique d'où découle la liberté d'appréciation, sans quoi le droit ne pourrait pas garantir la légitimité démocratique.

Les apports les plus originaux et les plus nouveaux de Moor on peut les trouver dans les contributions à l'étude de la norme. La thèse principale est formulable ainsi: la norme juridique est impuissante à maîtriser la réalité sociale, politique et économique si on la conçoit et on la dévisage comme un ensemble de règles générales abstraites prescrivant «des comportements identiques et égaux à un nombre indéterminé de situations individuelles ». L'attribution du sens par le système juridique implique la répétitivité de la reproduction et l'indétermination des cas à recouvrir. L'application de la norme ayant trouvé son sens dans le dit système, l'égalité formelle est alors assurée. Il arrive toutefois que la sélection des différences pertinentes soit irréalisable. Dans ces cas, afin de solutionner la question et garder la cohérence du système, il faut fabriquer un sens nouveau, donc produire une inégalité matérielle. Etant donné qu'il faut produire le sens toutes les fois que les nécessités concrètes l'exigent, la théorie juridique doit forcément osciller entre la logique déductive du normativisme et l'intuitionnisme du décisionnisme de l'action. La généralité et la juridicité de beaucoup de normes deviennent friables et la dilution de la souveraineté est imparable. Au même moment les égalités matérielles poussent à la marge les égalités formelles.

En réduisant l'application du droit à l'exercice d'un pouvoir, il est inévitable de dériver du principe d'égalité nombreuses logiques et différents modèles de 
juridicité. Si la norme n'est pas le fondement du droit mais son produit, une théorie du mode d'existence des relations juridiques abstraites et impératives, ne peut être que superflue.

Pour Moor le droit est la forme légitime d'exercice du pouvoir de la démocratie, la manière principale pour produire des «actes de pouvoir dont la validité est subordonnée à leur intégration dans un ordre de signification qui, quoique dépassant les auteurs, est accompli par eux». «L'organisation du système juridique comme travail de production de sens présuppose la double garantie de l'autonomie sociale et de l'autonomie individuelle en même temps qu'elle les conditionne réciproquement». Le droit, système poiétique, mieux autopoïétique, est donc structuré par la logique textuelle garante du principe de rationalité. Toutefois il est précisé que «la logique textuelle ne peut faire autrement que de se servir du métatexte pour établir les textes. Dans leur intégration à l'ordre juridique comme système, de même que, pour la même raison, l'élaboration des normes, individuelles et générales, doit puiser dans ce réservoir». Cette «logique textuelle est un principe d'organisation qui peut avoir à se manifester même comme supérieur à lui-même, puisque, garantissant une exigence générale de rationalité, il peut conduire à écarter sa propre application.»

Toujours selon Moor, «le droit est [...] un langue que la société se parle à ellemême pour être en mesure de dénoter ce qui se passe en elle.» L'analyse de cette langue est essentielle et l'approche herméneutique du phénomène juridique devient alors indispensable pour l' «appréhension complète de la consistance de l'Etat de droit.» Bien entendu, l'herméneutique est un pouvoir, un mode d'exercice du pouvoir mais aussi une modalité pour le saisir et le rationaliser.

La conclusion de toutes ces analyses, de ces déconstructions conceptuelles, de ces constructions théoriques, est plutôt de nature philosophique. En effet, Moor se demande: «La justice est-elle effectivement rendue quand le droit est dit?»; «Le droit peut-il vraiment se passer d'une conceptualisation de la justice?»

La réponse à ces deux interrogatifs est en cohérence absolue avec les élaborations conceptuelles précédentes. La justice est une valeur complexe historiquement située, elle est la revendication d'un ordre nouveau, elle est conflictuelle car traversée d'antagonismes et de contradictions, elle est autorité, elle n'est pas dans le droit: «[...] il n’y a d'action juste que si elle est considérée comme juste, que si elle est elle-même jugée par ces instances anonymes que sont la conscience universelle, la mémoire collective, le tribunal de l'histoire, les récits légendaires ou la reconnaissance des philosophes. [...] la justice est en même temps la sentence et son fondement; elle n'est pas parole, mais acte qui se dicte à soi-même son propre sens; elle est pour elle-même sa propre autorité».

Contrairement au droit où tout litige doit être tranché par des autorités compétentes et tout conflit exige une solution s'imposant à tous les acteurs au titre de textes normatifs contraignants et produisant une très forte clôture sémantique, la «justice n'a ni autorités ni textes normatifs ». Ouverte à tout vent, la justice est foncièrement dialogique. Mais lorsqu'elle est mise en forme par la loi ou par la sentence, elle abdique à ses potentialités et à ses virtualités et devient droit par transsubstantiation. Aussitôt que let droit est dit, «la norme juridique sera l'objet de nouveaux discours de justice qui, niant la justice réalisée, imagineront d'autres projets, toujours et encore plus justes.» Pour toutes ces raisons, le droit ne sera jamais la justice et celle-ci jamais le droit. 
Bien que fonctionnant de façon autonome, la justice et le droit sont interdépendants. C'est bien cette interdépendance à constituer les fondements d'une société juste et historiquement déterminée La conclusion de Pierre Moor a la saveur d'un apophtegme: "Le droit est l'ordre de la justice, mais elle s'y pervertit; la justice est le désordre du droit, mais il s'y renouvelle.»

4. Le résumé des recherches de Moor en épistémologie juridique montre, je l'espère, leur richesse foisonnante, l'originalité de certaines approches, prometteuses, engageantes, mais aussi la densité et la complexité des questions traitées, certaines desquelles suscitent, bien entendu, des perplexités.

Que le droit soit une «usine» à fabriquer du sens, et à le rendre plausible grâce à différentes formes de représentations, qu'il contribue à rationaliser les comportements, à gérer les conflits, à étalonner la violence, personne ne peut en disconvenir. Il est évident qu'il s'agit d'un processus dans le temps, assuré par le travail de différents acteurs hiérarchiquement ordonnés, et qu'il produit comme résultat l'unité et la continuité de l'ordre juridique.

Que le passage de la formulation abstraite et générale de la norme à l'application concrète soit possible grâce au travail inventif, à l'esprit innovant, voir créateur, à l'œuvre «de lecteurs» qui utilisent les ressources du système et extra système, qui les accroissent et les étendent moyennant l'interprétation et à l'argumentation, Moor l'a démontré avec maestria, notamment en mettant en évidence la pesée de la «Law in action» sur la «Law in books», selon la terminologie de R. Pound.

J'adhère également à sa conception que le droit est une forme de la société, qu'il ne faut pas le réduire à la règle de droit, à un prescripteur de conduites, qu'il est plutôt un «modèle» portant davantage sur la situation que sur la conduite. J'aurais aimé toutefois qu'il précise avec une grande netteté que l'interprétation ne relève pas de la logique mais de cette forme particulière de raisonnement qui cherche à obtenir la persuasion. J'aurais souhaité qu'il dise explicitement que le droit est le produit d'une histoire, de conflits sociaux, de déchirements idéologiques, changeants, relatifs, que ses évaluations sont inférées des prémisses du législateur mais aussi des préférences idéologiques et des orientations culturelles de l'interprète.

Certes, pour faire du sens dans le monde juridique, pour y introduire de l'ordre, de la cohérence et de l'intelligibilité, il faut postuler que le sens en tant que réalité publique et la rationalité soient liés. Mais comment expliquer la production et la détermination du sens en l'absence d'une structure génératrice? Peut-on ignorer que n'importe quelle signification se constitue tout d'abord par rapport aux conditions socio-historiques et aux rapports intersubjectifs d'une époque et d'une société données? L'herméneutique (dans la version Hans-Georg Gadamer ou dans la version Jürgen Habermas) et la théorie de l'argumentation de Chaïm Perelman ne donnent pas des réponses plausibles à toutes les questions soulevées par la théorie et la pratique du droit. Je doute que ces théories arrivent à saisir le signifié de la norme, à en garantir la pertinence et la cohérence narrative, et surtout à incorporer les constructions nouvelles dans le système de l'ordre juridique préexistant et construit à partir de valeurs majoritairement acceptées et de stratégies ouvertes.

L'élucidation du «mystère» des rapports existants entre l'interprétation et l'argumentation, entre la codétermination du sens par le juge et la prédétermina- 
tion par le législateur, me parait un passage obligé pour effacer des tels doutes. Je partage la thèse de Moor selon laquelle les théories étatistes et impérativistes, les théories formalistes et légalistes de la justice (ces dernières affectées par une idéologie politique) sont insuffisantes et insatisfaisantes car elles négligent le fait que les normes juridiques sont des valeurs antécédentes aux actions, que le droit est un discours évaluatif utilisant des typologies normatives fixes (nullité, décadence, annulation, révocation, etc.) et des typologies sociales très variables, que les énoncés des juristes sont tous descriptifs. J'admets également que les formulations des interprètes, en quête du sens des mots et des énoncés, sont exprimées en un métalangage. Cependant je reste de l'avis que le langage-objet de ce métalangage est prioritairement celui du législateur et de son ratio legis, sans quoi il serait impossible d'avoir les critères établissant l'appartenance à l'ordre juridique et d'identifier la validité formelle et la validité matérielle.

S'il est vrai qu'aucune conclusion normative ne peut être inférée d'une série de prémisses descriptives (Hume), alors il est difficile de ne pas prendre en considération l'autonomie du langage juridique avec ses discours descriptifs (vrais/faux), ses discours prescriptifs (normatifs) et ses discours évaluatifs (dénués de toute valeur de vérité). En plus, je doute qu'on puisse faire l'économie de la donnée selon laquelle le langage prescriptif ne transmet jamais d'informations ni de jugements sur la réalité mais seulement des ordres prescriptifs (ni vrais/ni faux, aucune valeur de vérité, validité évidente) afin de diriger, de conditionner, de modifier un comportement présumé ou possible, d'obliger un sujet à en suivre le commandement?

Pendant longtemps le droit, garant de la paix social et de l'ordre économique et social, a été conçu et perçu comme un ensemble d'obligations et d'interdictions corroborées par des contraintes et des coercitions. Mais dès les années '60 le «Welfare State» a commencé à intervenir dans les processus de production et de distribution des richesses. De conséquence, en plus du contrôle social et de la répression, il a dû s'arroger des fonctions de direction et de promotion d'une multitude d'interventions sociales. Pour exercer ces fonctions avec efficacité, il a ajouté aux sanctions négatives (la peine, le dédommagement, etc.) une série de sanctions positives (primes, incitations, conseils, exhortations, commandements). Dès lors le système juridique administratif (mais d'une manière moins marquante également tous les secteurs du droit public) s'est trouvé confronté à des problèmes interprétatifs d'une difficulté de plus en plus croissante, à l'impossibilité de distinguer la structure et la fonction du droit, à la constatation que l'ordre juridique peut servir n'importe quelle finalité, qu'il est apparemment dépourvu d'une finalité propre, spécifique.

La nature de ces règles est d'une complexité et d'une complication exceptionnelles et pourtant le travail sur les ordres normatifs arrive à mettre en évidence qu'en droit administratif beaucoup de jugements sont plus proches des jugements d'équité, de l'expérience sociale, du contexte historique que de la jurisprudence traditionnelle ou ordinaire. Il est incontestable que pour rendre compte de la réalité sociale, de la transformations des rapports sociaux d'aujourd'hui, de la croissance de l'Etat social, des privatisations et de bien d'autres phénomènes sociaux, le droit administratif à dû recourir aux facteurs extra juridiques, lesquels constituent les matériaux (contextualisés) informatifs primaires indispensables à la mise en forme des énoncés narratifs à la base des performances interprétatives 
et puis des synthèses prénormatives ou quasi-normatives. Par la surdétermination du sens obtenu au moyen de la contextualisation, on parvient plus aisément à la reconstruction narrative de la cohérence de l'ordre juridique.

Ceci reconnu et admis, il n'en reste pas moins que l'autonomie relative et la spécificité de ce langage prescriptif demeurent des questions importantes et dont il est périlleux de se débarrasser sans en débattre. Peut-on négliger que le juriste a surtout à faire avec le monde de l'expérience juridique, avec les règles des comportements et pas avec des comportements? Peut-on méconnaître que les propositions normatives établissent comment il faut et on doit agir en des circonstances données? Et que ces propositions visent des comportements possibles et probables et pas des comportements effectifs et réels?

Ce travail implique que le juriste doit interpréter à la fois le discours du législateur, la structure de l'ordre juridique, les théories du langage prescriptifs, les procédures utilisées pour la reconstruction du contexte, les conflits en jeu et puis il doit recomposer le tout en un récit narratif aboutissant inévitablement à une norme.

Il me semble qu'un tel travail présuppose, certes, une métathéorie mais aussi une théorie rendant compte moins des contenus que des caractères formels ou structurels de ce travail relativement autonome de production et d'extinction des normes, de solution des conflits entre modèles normatifs. En faisant abstraction des contenus de l'expérience, travaillant sur les aspects formels de cette même expérience, l'objet du travail consiste à la reconstruction de ce qu'est prescrit et à distribuer des qualifications normatives (obligatoire, interdit, permis, etc.,) à certains comportements. Ce travail donne naissance à une théorie autonome, cumulative, susceptible de servir à la constitution du paradigme de la science du droit en action.

Le cœur, mieux le noyau dur d'où tout provient, est, dans l'enquête épistémologique de Moor, la politique en tant que représentation de la société par ellemême. Cette définition est un euphémisme par litote. En réalité il aurait été plus ponctuel d'écrire «le pouvoir». Or cette notion renvoie à un prédicat si universellement vague que n'importe quel sujet individuel peut y être subsumé. Toutes les relations sociales, de n'importe quel type et de quelle nature, sont dissymétriques. Est-il utile et profitable de les imputer à un micropouvoir ou à une micropolitique?

Je n'ai pas oublié que Moor précise régulièrement qu'il définit la micropolitique comme étant «la part de liberté dont jouit, à quelques niveau que ce soit, n'importe quelle instance étatique, dès lors qu'elle est dotée d'un pouvoir qu'elle peut faire peser sur 'ses administrés'. L'exercice concret de la puissance publique, dans quelques situation que ce soit, est la manifestation d'un pouvoir politique et requiert à ce titre une légitimation pour être acceptable et, dans la mesure du possible, accepté.»

Même en laissant de côté le fait qu'il y a nombreux pouvoirs étatiques non disciplinés par la loi et ne nécessitant pas une légitimation, je reste convaincu que l'autonomie relative de l'ordre juridique ne peut pas être déclassée. Il me semble que les mécanismes de cette autonomie relative sont très différents de ceux de la politique, que les mécanismes dont se sert la fabrique du droit sont soumis à des révisions et à des corrections non imposées par les rapports de force et par la règle de la majorité. La reconnaissance de l'autonomie relative du droit permet de 
concevoir une science du droit systématisant la jurisprudence, le travail interprétatif et argumentatif des juristes ainsi que leurs apports au délinéament du concept de pouvoir et à la définition de la politique de l'Etat dans la société globalisée. Ainsi on pourrait finalement ne plus entendre que le pouvoir est une entité physique, qu'il se trouve partout et se caractérise comme une «microphysique».

5. Le droit est une discipline socio-historique ayant toutefois des approches aux phénomènes sociaux différentes de celles des sciences historiques et sociales. Alors que ces dernières disciplines partent de l'observation des actions et des comportements sociaux tels qu'ils sont pour arriver ensuite à identifier les normes les ayant régis, le droit postule qu'une règle donnée est à la base d'un comportement qu'il doit qualifier de licite ou illicites, etc. Dans les sciences historiques et sociales l'observation/description est toujours plus importante que l'interprétation, en droit c'est exactement le contraire.

Que l'interprétation juridique fasse de plus en plus recours à l'histoire, aux sciences sociales et à la philosophie, qu'une telle interdisciplinarité n'arrive pas à effacer la différence des approches et les finalités des diverses disciplines, c'est tout à fait dans l'ordre des choses. Cette diversité est une des façons d'exprimer la complexité des phénomènes sociaux, de souligner la distinction entre ce qui est et ce qui doit être, entre les valeurs et l'équilibre social. Le travail juridique constituant la science humaine du droit met en forme les valeurs, les fait passer au crible de la raison et les rend pour ainsi dire légitimes dans un contexte donné.

Cette science humaine du droit a une capacité d'auto-organisation inconnue aux autres sciences de l'homme et de la société. Elle arrive à rendre compte de façon cumulative des interactions, des transactions entre les agents, des phénomènes aléatoires, des technologies nouvelles et des structures émergentes, comme d'un système naturel que l'on va observer de l'extérieur. Cela permet une analyse dans un certain cadre posé à l'avance, favorise la rationalisation des rapports sociaux, assure le maintien d'un certain équilibre social, modère les actions de la puissance publique.

L'épistémologie juridique de Pierre Moor suscitera, au fil des années à venir des débats intellectuels serrés, mais elle d'ores et déjà me parait une invitation adressée aux sciences sociales à s'intéresser davantage au droit, à mieux connaître ses techniques de recherche, à ses façons de représenter les phénomènes. Pierre Moor nous apprend que le travail d'une science, soit-elle normative, présente un avantage particulier, celui de l'apprentissage permanent de la méthode critique. Elle conduit à prendre la distance par rapport à n'importe quel dogme ou quelle valeur. Ce qui conduit à faire l'expérience d'un grand relativisme par rapport aux croyances et aux certitudes dans la vérité de la science, sans toutefois succomber sous le poids du scepticisme qui proclame qu'il n'y a rien de vrai, que l'on ne peut croire en rien du tout.

La lecture des travaux d'épistémologie juridique de Pierre Moor nous apprend, en outre, que les vérités sont relatives aux domaines, aux lieux, aux temps dans lesquels elles sont exprimées et aux règles qui sont utilisées pour les établir. Dans cette perspective les règles de droit (sans oublier celles de l'éthique et de la morale) nous aident à entrevoir ce qui favorise le développement d'une vie sociale plus ou moins harmonieuse.

Pour ces raisons Pour une théorie micropolitique du droit est un événement culturel d'une importance notable, notamment pour la révision des relations entre 
le droit et les sciences sociales, pour la reconnaissance du droit et de la jurisprudence en tant que science storicosociale.

\section{ADRIEN NAVILLE
ÉPISTÉMOLOGUE ET PHILOSOPHE SOCIAL}

Né en 1845 dans une famille patricienne genevoise très attachée à la tradition du protestantisme libéral, Adrien Naville, depuis sa plus tendre enfance, a vécu dans un milieu intellectuel ouvert et vif, pour lequel l'existence d'un lien fort entre la religion, la philosophie et la science était conçu et perçu comme le problème central de la réflexion intellectuelle, de l'engagement moral et de l'éthique tout court ${ }^{9}$.

Pour son père, Ernest, - philosophe, théologien, réformateur politique, auteur d'un système philosophique ancré dans la tradition protestante calviniste mais pétri d'idées politiques et principes libéral-démocratiques, biographe, interprète d'œuvres philosophiques et littéraires, pédagogue, éditeur des écrits de Maine de Biran-, la raison est compatible avec la foi dans la transcendance, la science avec la philosophie et celle-ci avec la religion.

Formé culturellement par son père, influencé par l'exégèse paternelle de la «Weltanschauung» et du système philosophico-religieux de Maine de Biran, Adrien Naville lit et relit les Nouvelles considérations sur les rapports $d u$ physique et du moral de l' homme (1834) et surtout l'Essai sur les fondements de la psychologie (1859), où le problème de l'habitude, en tant que révélateur psychique, est présenté comme le mécanisme même de la faculté de penser. L'habitude permet de distinguer la sensation de la perception, l'affectif de l'aperception, et de fonder sur les différents sens, selon le degré d'activité qu'ils mobilisent, des analyses complètes de la causalité, d'élaborer une solide philosophie spiritualiste. La thèse anthropologique du Mémoire sur l' habitude de Maine de Biran de la scission activité-passivité, de la vie animale inconsciente, de la vie humaine consciente, de la vie de l'esprit culminante dans l'extase de l'infini, sera, au fil des années, reprise, approfondie, modifiée, mais elle restera une constante de sa réflexion, le thème, explicite ou implicite, à la source de tous ses premiers travaux.

Les critères pour rapprocher les sciences à la philosophie et à la religion, Adrien Naville va les demander, tout d'abord, à la recherche historique, à l'interprétation des grands textes philosophiques, aux études anthropologiques et psychologiques. Et bien entendu à la religion, laquelle a instauré et renforcé la morale; la protection de la propriété privée, de la famille. En régentant les comportements humains réguliers et corrects, la religion a joué un rôle capital dans le développement de la vie sociale et des institutions de régulation et de contrôle des rapports entre les hommes et entre les hommes et les choses. De cette

Les rapports de la science avec la religion ont été toujours au centre des préoccupations des savants genevois. Des renseignements à ce propos se trouvent dans le recueil Les savants genevois dans l'Europe intellectuelle du XVIII ${ }^{e}$ au XIX ${ }^{e}$ siècle, éd. par Jacques Trembley, Genève, Ed. du Journal de Genève, 1987, ainsi que dans le volume de J. Starobinski, Table d'orientation. L'auteur et son autorité, Lausanne, L'âge d'homme, 1989. 
façon elle a contribué à former des hommes responsables, à amplifier en eux le sens de l'intérêt, l'expérience de la réciprocité des droits et des devoirs, du commandement et de l'obéissance.

\section{Les premiers écrits}

Avec ses premiers écrits, Naville essaie de rendre compte du processus de formation dans l'histoire des valeurs ${ }^{10}$, de comprendre pourquoi les controverses autour du principe de nationalité suscitent autant de passions et si ce principe s'applique ou non à la Suisse ${ }^{11}$. Ces controverses lui paraissent dévoiler la crise spirituelle affectant la conscience européenne ${ }^{12}$. Il en cherche les annonces et les remèdes chez des personnalités hors série, portraiturées avec un bienveillant doigté $^{13}$. Il est convaincu que les luttes ayant déchiré l'unité de la Chrétienté, ont rendu les hommes injustes envers leurs adversaires, incapables d'en apprécier les raisons et les mérites, insensibles aux supplications et à la douleur des ennemis ${ }^{14}$.

Sa licence universitaire en théologie, en 1872, traite, sur la base d'une lecture empressée des écrits de Saint Augustin, du rapport entre la science et la foi, entre la morale et le mal, au moment du passage d'une époque à une autre. La foi chrétienne, étrangère à l'intellectualisme, au rationalisme, à l'idéalisme, a toujours eu pour objet et finalité essentiels la vérité. La distinction de la science moderne entre le monde sensible et le monde intelligible, entre les sens et les abstractions capables de les représenter, donne une place prééminente aux facultés intellectuelles, et ainsi elle sous-évalue les facultés morales et confond le bien avec l'être, le mal avec le non être. Ne reconnaissant pas que la foi est davantage qu'une simple adhésion de l'intelligence aux faits, la science moderne oblitère le libre arbitre et dévalorise la liberté, conçue à la fois comme liberté naturelle (les individus ont la capacité naturelle d'être libres), comme liberté acquise (l'individu peut développer encore plus la capacité naturelle) et comme liberté circonstan-

${ }^{10}$ A.Naville, Histoire de la Société de Zofingue après 1832, «Feuille centrale de la Société de Zofingue», VI, 1865, n. 4, pp. 117-125; n. 6, pp. 173-182; n. 7, pp. 205-212.D'après Th. Dufour, Catalogue des membres de la Section genevoise de la Société de Zofingue, Genève, Fick, 1867, p. 34, Naville a été admis dans la Société, président Charles Martin, le 30 novembre 1864 et en est devenu président pour la période 1866-1867.

${ }^{11}$ La nationalité suisse, Ibid., VI, 1866, n. 8, pp. 243-249: «Seule, en Europe, la Suisse représente un grand principe politique: la démocratie républicaine et fédérative. [...] ce qui donne à notre développement national sa force et son originalité.» «Que d'autres peuples cherchent leur nationalité dans le fait de la communauté du langage ou de la disposition des frontières, la notre est fondée sur des souvenirs communs de liberté et sur des efforts communs pour la réalisation d'une grande idée.»

${ }^{12}$ L'art dramatique à Genève au XVI siècle, Ibid., IX, 1969, n. 5, pp. 137-146; n. 6, pp. 169-178.

${ }^{13}$ Par exemple, Le comte Agénor de Gasparin, «Journal de Genève», 16 mai 1871, et puis en tiré à part, Genève, Ramboz et Scuchardt, 1871, 9 pp. Ou encore Alfred Gilliéron, «Feuille centrale de la Société de Zofingue », XIII, 1873, pp. 77-80; Pestalozzi, «L'Educateur», XII, n.9, $1^{\text {er }}$ mai 1876, pp. 129-134, repris avec le titre La doctrine psychologique de Pestalozzi, «Aujourd'hui. Feuille d'art et d'éducation», III, n. 2, février 1919, pp. 17-21. Voir aussi la brochure En souvenir d'Arthur King, 1844-1921, Genève, Kundig, 1921.

${ }^{14}$ Ces thèmes sont traités dans l'étude Les catacombes romaines, «Le Christianisme au XIX ${ }^{\mathrm{C}}$ siècle », 21 mars 1873, p. 58; 28 mars 1973, p. 61; 4 avril 1873, p. 68; 18 avril 1873, p. 78. 
cielle (l'individu peut parfaire ses capacités en certaines circonstances et selon des modes variables) $)^{15}$.

Est-ce que la négation de la foi dans le surnaturel est un phénomène spécifique à la modernité? Est-ce le produit des progrès de la science, de ses découvertes, des philosophies positives, ou bien s'agit d'un retour à de conceptions abandonnées, de la renaissance de doctrines anciennes sous l'impulsion de facteurs particuliers ? ${ }^{16}$

A ces questions Naville donne une réponse dans le livre sur Julien l'Apostat, l'Empereur neveu de Constantin Ier le Grand, à l'origine de la revitalisation des philosophies néoplatoniciennes, promoteur du retour à la tolérance religieuse et animateur des courants prônant la recrudescence du polythéisme et du paganisme. L'analyse des écrits de Julien, et notamment du traité Adversus christianos, réfuté par Saint Cyrille d'Alexandrie, tient compte aussi de leur réception et des interprétations dans et par la culture occidentale postérieure. Ce livre, malgré son programme explicite de révéler l'inconsistance des doctrines exposant l'impossibilité d'un accord entre la foi et la raison, les contradictions du monothéisme et les précarités du polythéisme, ne va pas au-delà de la modeste enquête intellectuelle, érudite et informée, mais peu novatrice ${ }^{17}$. Cependant une lecture attentive suscite l'impression que l'auteur veuille remplacer la déduction kantienne (les conditions qu'une catégorie doit posséder pour avoir une valeur objective) par l'interrogation portant sur la signification et la valeur des énoncés. En réduisant la théorie scientifique à l'expérience, Naville, sans le dire, réduit la déduction à l'explication et les conditions de l'objectivité de l'expérience à celles de la construction des référents du langage, mais il ne dit pas à quelles conditions la science pourrait être compatible avec la religion.

Dès sa nomination, en 1876, à la chaire de philosophie et d'histoire de la philosophie de l'Académie de Neuchâtel, Naville s'attache à étudier les habitudes, les associations psychologiques, la faculté d'abstraire et de généraliser, la genèse psychologique des théories ainsi que la doctrine selon laquelle ce sont uniquement les faits à valider les concepts. Ses études visent à remplacer la synthèse a priori de Kant par une science psychologique propédeutique d'une théorie de la connaissance des individus, de leurs sensations, représentations, intuitions et de leur fantaisie. L'analyse des mécanismes associatifs psychologiques et de la genèse des sensations, des représentations et des idées trouve sa raison d'être dans la décision de se distancer du néo-kantisme positiviste, de fixer les limites de la raison humaine, de réagir et s'opposer à certaines tendances philosophiques qui dévalorisent la science et rejètent, au nom de l'affectivité et de la volonté, toutes les formes de rationalité. Le refus des développements philosophiques de la théorie de la relativité, du nouvel atomisme et du darwinisme est sans appel.

Naville connaît les Logische Untersuchungen de Trendelenburg, les Elemente der Psychophysik de Fechner, le System of Logic de Mill, l'œuvre Mikrokosmos de Lotze, les travaux sur la physiologie des sensations d'Ernst Weber, mais il en trie

${ }^{15}$ Saint Augustin. Etude sur le développement de sa pensée jusqu'à l'époque de son ordination, Genève, Rambot et Schuchardt, 1872. Naville revient sur cette problématique avec l'étude Saint Augustin d'après Carl Bindemann, «Revue de théologie et de philosophie», IX, 1876, pp. 414-444.

${ }^{16}$ La négation du surnaturel est-elle moderne, «Revue chrétienne», 1874, pp.643-658.

${ }^{17}$ Julien l'Apostat et sa philosophie du polythéisme, Paris, Sandoz et Fischbacher, 1877. Une réimpression anastatique a parue à Rome, L'Erma di Bretschneider, 1972. 
les idées avec le tamis paternel. Oui, il faut étudier les phénomènes psychiques de la même façon que les phénomènes naturels, mais la spéculation philosophique et la religion restent les formes supérieures de la connaissance, celles capables de donner du sens universel à la totalité.

L'étude scientifique des sentiments débute avec un essai consacré à la spécification de l'amour propre, sentiment indirecte et dérivé, produit spécial de l'amour de soi, «plaisir durable, permanent, qui nous suit partout, qui se mêle à tout, qui anime tout de sa lumière et sans lequel la vie extérieurement la plus heureuse serait intérieurement insupportable.» ${ }^{18}$

Convaincu que l'homme porte en lui naturellement le développement esthétique, intellectuel et moral, que l'éducation doive tendre à développer par l'exercice les virtualités naturelles ${ }^{19}$, Naville ébauche, dans le discours rectoral d'octobre 1883, une première, rudimentaire classification des arts, de la connaissance empirique, de la connaissance scientifique et plus particulièrement du rapport entre la théorie et la pratique ${ }^{20}$.

Avec l'activité pratique nous transformons la réalité; moyennant l'invention et l'exécution nous obtenons la réalisation de nos projets et de nos idéaux. L'activité théorique nous permet de prendre connaissance de la réalité alors que l'art cherche à rendre les choses conformes à la pensée. La science, en révélant les mécanismes de la nature et ses lois, consent de prévoir les conséquences des actions humaines. Toutefois elle n'arrive pas à satisfaire tous les besoins et les attentes des hommes. «La science conçoit l'ensemble des choses comme un immense mécanisme dont l'homme n'est qu'un rouage. Mais cette conception décourageante pour la volonté aboutit, lorsqu'elle est conduite jusqu'à ses limites extrêmes, à des contradictions que l'intelligence ne peut accepter.» ${ }^{21}$

Dans l'essai consacré à l'attention et à la distraction, Naville ajoute que les facteurs primordiaux de la recherche scientifique sont la curiosité intellectuelle et la volonté de connaître. Pour cette raison, nous percevons les objets auxquels nous nous intéressons. L'attention oriente et scelle les convictions et les attitudes. Les divergences d'opinions dérivent du fait qu'on immobilise l'attention sur des faits différents et/ou on les interprète diversement et inégalement. "Aucun individu n'a le droit de se croire l'homme complet; il n'y a d'autres hommes complets que l'humanité.» Par conséquent, «La tâche de chacun de nous n'est ni de tout faire, ni de tout comprendre, mais seulement de contribuer pour sa part à l'œuvre humaine collective: l'accroissement progressif du bonheur et de la vérité.» ${ }^{22}$

${ }^{18}$ L'amour propre. Etude psychologique, «Revue philosophique de la France et de l'Etranger», VI, 1881, pp. 652-662.

19 Voir à ce propos l'article Pestalozzi, «L'Educateur», XII, n. 9, 1r mai 1876, pp. 129-134.

${ }^{20}$ La Science et l'Art. Discours, Neuchâtel, Borel, 1883. Il revient sur cette question dans divers comptes rendus (à H. Lecoultre, Essai sur la psychologie des actions humaines d'après les systèmes d'Aristote et de Saint Thomas d'Aquin, Lausanne, G. Bridel, 1883) in «Revue de théologie et de philosophie», mars 1885; pp. 236-239; in «Bibliothèque universelle et Revue suisse», XXXI, 1886, pp. 445-446, (à B. Labanca, Il cristianesimo primitivo, Torino, 1886) pp. 655-656 et dans la « Revue philosophique de la France et de l'Etranger», IX, 1884, pp. 692-699.

${ }^{21}$ La Science et l'Art, op. cit., p. 11.

${ }^{22}$ Attention et distraction, «Bibliothèque universelle et Revue suisse », XXX, 1886, pp. 5-29. Les citations se trouvent à la p. 28 et à la p. 29. 
L'action de rire est traitée comme une «arme de la raison», un instrument utile pour la cure de certaines maladies morales ${ }^{23}$ alors que la connaissance par les sens et l'expérience ainsi que le schéma psychologique du raisonnement inductif, la nature et la valeur de cette opération intellectuelle, suscitent quelques perplexités ${ }^{24}$.

L'induction postule un axiome selon lequel un même ensemble de causes produit nécessairement un même ensemble d'effets, ou encore que si le même ensemble de causes se répétait, il produirait forcément le même ensemble d'effets. Naville affirme que cet axiome est conditionnel puisque fondé sur la supposition de l'existence d'une relation d'identité entre la répétition des causes et la production des effets. En plus, l'induction présume la présence d'une même identité pour plusieurs ensembles de causes. Les identités étant toujours partielles, associées constamment à des différences, comment peut-on délimiter l'objet auquel appliquer l'induction? Si la raison logique gouverne les rapports de dépendance et les lois sont des rapports nécessaires, alors l'induction ne produit que des généralisations abusives et des inférences conjecturales. Au $\mathrm{XX}^{\mathrm{e}}$ siècle, Karl Popper critiquera l'aspect non rationnel et trop intuitif de l'induction et en récusera, avec d'autres arguments, toute valeur dans la recherche de la connaissance.

Naville distingue «la raison hors de nous», ou raison logique, et « la raison en nous », ou raison morale. La première repère et traite les rapports de dépendance, innombrables et de genres divers, entre les choses, tandis que la seconde est la faculté de comprendre les rapports de valeurs. Il revient souvent sur ces thèmes pour préciser que les hommes élaborent deux sortes de représentations, celle des objets et des phénomènes matériels et celle des états et des événements psychiques ou spirituels. Pour cette raison il faut faire une nette distinction entre la beauté organique, la beauté sensible et la beauté expressive. «Entre nous et les corps en présence desquels nous sommes, il y a un intermédiaire, l'intelligence »; elle « intervient encore en nous représentant les impressions des âmes de ce corps. [...] Et parmi ces intermédiaires, une physiologie plus avancée discernerait, je n'en doute pas, le phénomène cérébral qui correspond à la compréhension d'un organisme et celui qui correspond à la représentation de certains états dans l'âme d'autrui.» ${ }^{25}$

Une vie intellectuelle et morale bâtie sur «le sable d'un idéal arbitraire » risque de condamner la société à la décadence. Pour échapper à une telle catastrophe il est indispensable de consolider «toujours plus solidement l'autorité de la raison sur le roc de la nature éternelle $»^{26}$, de ne pas oublier que le présent est pour la

${ }^{23}$ Le rire, «Bibliothèque universelle et Revue suisse», XXXII, 1888, pp. 6-30.

${ }^{24}$ L'induction a été refusé par la «Revue philosophique de la France et de l'Etranger», en 1888. Le manuscrit appartient au professeur Gabriel Aubert lequel m'en a facilité la consultation.

${ }^{25}$ La beauté organique. Etude d'analyse esthétique, «Revue philosophique de la France et de l'Etranger», XVII, 1892, II, pp. 182-191 et Beauté organique et beauté plastique, Ibid., XVIII, 1893, I, pp. 287-289. Le même thème est abordé aussi dans Lois physiques et lois psychiques, Ibid., XIX, 1894, II, pp. 402-404.

${ }^{26}$ La raison. Etude familière, «Bibliothèque universelle et Revue suisse», L, 1891, pp. 5-30. Naville, comme d'ailleurs les principaux penseurs romands, sont convaincus que la démocratie implique le danger d'une dictature de la majorité, de la suppression des libertés et de la propriété privée au nom de l'égalité sociale. Son adhésion aux doctrines éthico-politiques de l'auteur de La philosophie 
science une simple limite, un point mobile sur la ligne du temps, que la faculté de percevoir le passé est une des conditions sine qua non de notre intelligence et de notre manière d'être au monde.

Mais peut-on concilier l'optimisme métaphysique et le pessimisme expérimental, la raison qui affirme le bien et l'expérience qui constate le mal? Quel est le rôle de l'art dans et pour le progrès moral de nos sociétés ? ${ }^{27}$

La raison pose les fondements de la morale, de la croyance à l'obligation et à la liberté morale, comme amour créateur, comme principe de l'unité de l'être; elle indique à la fois les dangers du déterminisme et de l'athéisme ainsi que la nécessité de la solidarité morale et sociale, réquisits indispensables afin que les individus isolés deviennent des personnes libres et responsables.

L'insuffisance des philosophies actuelles (le réalisme, le naturalisme, l'idéalisme, l'irrationalisme, le matérialisme, le spiritualisme athée et agnostique, le sociologisme) serait une des causes majeure du chaos régnant en Europe. Ces théories ne comprennent pas qu'à des objets différents il faut des méthodes différentes, qu'il ne faut pas condamner, par exemple, les faits psychiques inconscients parce qu'ils ne sont pas observables directement. Un exemple paradigmatique Naville le trouve dans la philosophie d'Herbert Spencer. Les idées générales de l'Anglais lui paraissent toutes dépourvues de cette hétérogénéité proclamée comme étant la loi nécessaire du progrès. Sa philosophie et sa sociologie mélangent les lois universelles et nécessaires avec les faits historiques plus ou moins généraux. L'explication scientifique proposée fait appel aux lois et à des faits antérieurs, comme si ces derniers fussent les produits de lois nécessaires ou de lois historiques. En plus, ses idées sur la religion sont contestables à tous les points de vue $^{28}$.

A la thèse que les lois expliquent tout, Naville oppose celle que les lois seules n'expliquent absolument rien, qu'il n'y a pas de loi nécessaire du progrès et qu'une loi n'est que la virtualité d'un rapport conditionnellement nécessaire. Les lois naturelles, à elles seules, n'expliquent pas l'ordre de l'univers, ses symétries, ses rythmes, son organisation. Alors que l'ordre change, se transforme, évolue, la science postule qu'après des intervalles réguliers il revient à des états identiques car, pour elle, les lois les régissant sont constantes.

\footnotetext{
de la liberté (1848-1849), de Le principe de la morale (1884), de La civilisation et la croyance (1887), Droits de la femme (1888) et Droits de l' humanité (1890), est prouvée par nombreux textes consacrés à ce penseur vaudois («...il est un de ceux dont les écrits m'ont le plus aidé, le plus soutenu dans les crises redoutables de la pensée»). Pour tous cf. Charles Secrétan, 1815-1895, «La Semaine littéraire», n. 57, 2 février 1895, pp. 49-51 mais aussi le compte rendu, in «Revue philosophique de la France et de l'Etranger», XXXVII, 1912, I, pp. 421-426, d'une biographie du penseur vaudois dont il apprécie surtout la conception de l'unité naturelle de l'humanité, fondement du devoir de solidarité («Le premier devoir que m'impose la solidarité, c'est de contribuer à rendre les autres libres.»). A voir également La loi du progrès d'après un ouvrage récent, «La Semaine littéraire», XXIII, n. 1138, 23 octobre 1915, pp. 511-512.

${ }^{27}$ Remarques sur les rapports de l'esthétique avec la morale. Leçon académique, in «Feuille centrale de la Société de Zofingue», XXXV, 1894, pp. 145-162, texte republié en brochure, Genève, Kundig, 1894.

${ }_{28}$ Voir à ce propos $\mathrm{H}$. Spencer, Passé et avenir de la religion, «Revue philosophique de la France et de l'étranger», XVII, 1884, I, pp. 1-14.
} 
Naville reste convaincu que «La pensée moderne réclame un principe premier qui contienne les raisons à la fois de la nature permanente des choses manifestées par les lois et du plan selon lequel se développe progressivement l'organisation. ${ }^{29}$ Cette pensée moderne est foncièrement incapable d'accepter que dans tout événement il y a du surnaturel, de la création, que le surnaturel est constitutif de toute réalité, que l'explication implique une disposition préexistante, des lois et des initiatives mais que toute conclusion d'elle à une substance ou à une cause universelle est illégitime. Les dialectiques lui paraissent des moyens pour suppléer à l'incapacité des hommes de saisir directement et pleinement la réalité ${ }^{30}$.

\section{A la recherche d'une épistémologie des sciences}

En 1892 Adrien Naville est nommé à la chaire de logique, de méthode et de classification des sciences de la Faculté des lettres et des sciences sociales de l'Université de Genève. A partir de 1909 il assume l'enseignement de la philosophie générale et de classification des sciences. En 1896 il est élu doyen et chargé de réorganiser les enseignements de la Faculté des lettres et des sciences sociales $^{31}$.

Ami et collègue du linguiste Ferdinand de Saussure, beau-frère d'Ernest Roguin, professeur de droit à l'Université de Lausanne ${ }^{32}$, par l'entremise duquel il fait la connaissance de Léon Walras et de Vilfredo Pareto, Naville comprend promptement l'importance de la théorie de l'équilibre général et les bouleversements que cette théorie, indirectement influencée par la linguistique générale saussurienne, allait provoquer dans les sciences économiques et sociales.

Un texte paru en 1897 en est la preuve ${ }^{33}$. Naville constate d'emblée que Walras se propose de fonder la science des lois de la richesse et de l'appauvrissement, de construire une science économique pure, d'accorder l'utile avec le juste, de révéler la corrélation entre la division du travail et le développement de la personnalité morale. Il repousse la thèse walrasienne de la nationalisation du sol, dit sa perplexité à propos de la formule égalité des conditions/inégalités des positions; il écarte la réduction, sur la base de la physiologie et de la psychologie, de la

${ }^{29}$ L'ordre de la nature matérielle et son explication scientifique. Leçon universitaire, « Revue scientifique», XXXII, 18 mai 1895, pp. 3-23, et en brochure: Genève, Georg, 1895. 477-481.

30 J.J. Gourd.Les trois dialectiques, «Revue de théologie et de philosophie», XXX, 1897, pp.

${ }^{31} \mathrm{Cf}$. à ce propos G.Busino et S.Stelling-Michaud, Matériaux pour une histoire des sciences sociales à Genève (1873-1915), Genève, Droz, 1965. Par la suite cité Matériaux..., op. cit.

${ }^{32}$ N. Bobbio, Un dimenticato teorico del diritto: Ernest Roguin, in AA.VV., Studi in onore di Salvatore Pugliatti. IV. Scritti storico-filosofici, Milano, Giuffré, 1978, pp. 43-70, repris dans Diritto e potere. Saggi su Kelsen, Napoli, ESI, 1992, pp. 193-213. Ce texte a été traduit en français par Alain Dufour: Le vaudois Ernest Roguin, sociologue et théoricien du droit, « Revue européenne des sciences sociales », XIX, 1981, n. 59, pp. 121-140. Voir aussi G.Busino, Contributi alla storia del pensiero politico contemporaneo.I : Ernest Roguin e Vilfredo Pareto, «Cahiers Vilfredo Pareto», II, 1964, n. 4, pp. 189-210.

${ }^{33}$ Economique et morale, «Revue philosophique de la France et de l'Etranger», XXII, 1897, I, pp. 19-28. Ce texte sera publié peu de mois après avec le titre L'Economie dite politique et les sciences morales. Etude extraite de la "Revue philosophique» et précédée d'une lettre à M. Léon Walras, Genève, Georg, 1897. 
science sociale à la mécanique sociale. L'économie, indépendante à l'égard de la morale, n'est pas la science du bien, elle n'enseigne pas ce qu'il faut faire, mais seulement, à la limite, ce qu'il faudrait faire si la richesse était le but unique, suprême, des hommes.

Naville admet qu'on puisse envisager l'économie en tant que science des lois naturelles, que le domaine d'étude principal et exclusif de cette science soient les rapports de dépendances nécessaires entre les causes et leurs effets, entre des conditions et leurs résultats, cependant il conteste l'extension de cette conception à la science sociale et la réduction de cette dernière à une psychologie physiologique, à une mécanique sociale. La science sociale en tant que science morale ne se borne pas à constater des relations fatales, elle imagine un ordre des choses meilleur de la réalité existante; elle commande «de construire, d'ordonner, d'organiser, de mettre en mouvement les corps et les esprits pour réaliser l'idéal dans la mesure du possible. La théorie de la justice suppose que des préceptes soient observés en vertu des lois psychologiques, mais aussi que les matériaux fournis par la nature soient ordonnés selon des plans nouveaux. Après que l'intelligence a constaté le réel, la raison construit l'idéal». C'est pour cette raison que la formulation des préceptes catégoriques abstraits est de la compétence des sciences morales. De ces catégorèmes l'acteur social dégagera «un programme catégorique concret adapté aux circonstances historiques dans lesquelles il se trouve » à $\operatorname{agir}^{34}$.

Walras, le 5 janvier 1897, réagit ainsi: «Je me rends parfaitement compte des divergences qui peuvent exister entre nous deux; mais il est un point, capital à l'heure qu'il est, sur lequel je pense que nous serions d'accord: savoir la réaction à opérer au nom du point de vue naturaliste de l'ancien droit naturel contre le point de vue fataliste et déterministe naturaliste de la sociologie biologique. Et, au surplus, m'occupant en ce moment d'arrêter ma doctrine autant que faire se peut, je suis attentif à toutes les critiques susceptibles de me suggérer quelques corrections.» ${ }^{35}$

Le 4 avril 1897 Walras communique à Naville que la distinction entre norme logique et norme morale, la doctrine des rapports entre les faits et leurs conséquences, la thèse que l'élément moral domine l'élément physique, que la norme morale, d'origine religieuse, fixe la distinction entre le bien et le mal, le vrai et le faux, lui paraissent dissimuler un certain «mépris de la réalité physique», «un excessif altruisme», une «réaction métaphysique». En affirmant «Pour ma part, je suis résolu à viser [...] à la conclusion et à l'équilibre $»^{36}$, il clôt définitivement les échanges intellectuelles avec Naville.

Par contre, les échanges intellectuels avec Pareto vont devenir serrés et consistants. Dès le 11 janvier 1897 la discussion porte déjà sur l' «essence des choses » et sur le rôle de la psychologie dans les sciences, et notamment en économie ${ }^{37}$.

${ }^{34}$ Economique et morale, op. cit., p. 24.

${ }^{35}$ Correspondence of Léon Walras and Related Papers. Edited by William Jaffé, vol. II, 1884 1897, Amsterdam, North-Holland, 1965, p. 719.

${ }^{36}$ Ibid., II, p. 746.

37 V. Pareto, Euvres complètes. Tome XIX. Correspondance, 1890-1923, publiée par G. Busino, Genève, Droz, 1975, pp. 323-326 et Matériaux..., op. cit., pp. 113-115. 
Pour Pareto il faut se tenir à l'écart des recherches sur les essences des phénomènes et des enquêtes de la psychologie. L'histoire des sciences montre qu'il y a des progrès lorsque la recherche prend «pour base des principes secondaires». Pour l'étude des actions humaines ce ne sont pas «les désirs que nous devons considérer», mais les volontés, mieux: les actes potentiels. Ce sont les choix qui constituent les principes fondamentaux, l'axiome principal de l'économie pure. L'étude des forces qui déterminent la volonté et comment la volonté détermine les actes, cette étude ne relève pas de l'économie pure. Cette discipline ne cherche pas à savoir ce que c'est que la morale, la justice, etc.; elle se borne à étudier en bloc les forces constituant les sentiments des hommes en corrélation avec l'organisation sociale. Sans rien préjuger de la nature de ces forces ni du déterminisme et du libre arbitre, domaines réservés aux recherches psychologiques, l'économie pure est semblable à la mécanique rationnelle et l'homo oeconomicus fonctionne comme une balance, plus ou moins parfaite, pour peser ces forces.

Une telle position est très différente de celle des marginalistes anglais et ne tient pas compte de la persistance des biais cognitifs dans les choix des agents ni même des processus mentaux qui conduisent aux décisions réfléchies, voire même rationnelles. En tous cas, pour Pareto l'économie pure est dans l'incapacité de donner des préceptes pratiques ${ }^{38}$. Prenant en bloc les différentes forces, elle ne peut pas les pondérer ni même traiter les informations, la perception des risques et les mécanismes de la décision. Or, en science sociale les différentes forces doivent être séparées. Dans l'état actuel de nos connaissances cette opération est irréalisable, d'où le constat que «l'acte humain» est «la résultante d'un grand nombre de causes (besoins, désirs, morale, justice, etc.)», qu'il constitue le socle «pour continuer dans les déductions ». Sans cela, il n'y a pas de commerce intellectuel possible. A titre d'exemple, Pareto ajoute ceci: «[...] le terme de vérité que vous employez est un de ceux qui m'est fort obscur. Je ne le conçois guère qu'en ce sens: il y a deux ordres de phénomènes, l'un intérieur, l'autre extérieur. Lorsque le premier coïncide avec le second, nous disons qu'il est vrai. [...] Vous désirez la justice dans la vérité. Eh bien! prenons notre point de départ, à votre point d'arrivée. Que le sentiment de justice vienne du besoin de vérité ou d'autre part, il est de fait qu'il existe. Pour la science sociale, il me suffit de savoir comment précisément il se manifeste.» ${ }^{39}$ Et pourtant la science sociale reste encore à créer. Les études de Spencer ne traitent que d'une partie de cette science. En plus, «Je ne saurais admettre une identité entre l'étude de l'évolution et l'étude de la science sociale. [...] Je découvre peu à peu les défauts des différentes théories, mais il me manque de nouvelles théories à substituer aux anciennes. $\gg^{40}$

${ }^{38}$ V. Pareto, Euvres complètes. Tome XXX. Lettres et correspondances. Compléments et additions. Textes rassemblés, introduits et annoté par G. Busino avec la collaboration de V. Monnier, Genève, Droz 1989, p. 313, lettre à Francesco Papafava du 10 février 1897 à propos du texte Economique et morale: «Ho scritto al Naville che secondo me né la scienza economica né la scienza sociale possono dare precetti. Questi sono solo dati dall'arte. La meccanica non dà precetti, l'arte dell'ingegnere si. E gli ho pure detto che per me la scienza sociale è la storia naturale delle società umane. L'economia politica è, all'incirca, la scienza che studia le funzioni di nutrizione di quegli aggregati.»

39 V. Pareto, Euvres complètes, op. cit., XIX, pp. 327-328 et Matériaux..., op.cit., pp. 116-117.

4o Op. cit., p. 332 et Matériaux..., op. cit., pp. 117-118. 
Ce que Naville écrit sur la justice en tant qu'aspect de la raison semble à Pareto inacceptable; il est convaincu qu'un tel concept recouvre différentes catégories d'actions. Il y a des justices bonnes et des justices mauvaises, selon les critères utilisés pour les définir. En outre, il doute qu'on puisse déduire de l'observation une éthique absolue. "C'est, ce me semble, ce que vous voulez dire en observant qu'on ne peut pas extraire les principes de la théorie de la justice de la simple observation de l'âme humaine. Jamais cette observation ne vous donnera un impératif absolu. Elle pourra seulement vous dire: si vous voulez obtenir tel et tel résultat, voici la voie que vous devez suivre»; «[...] je ne puis m'empêcher de sourire quand je lis l'éthique que [Spencer] a voulu déduire de sa théorie de l'évolution. L'expérience ne vous donnera jamais l'absolu, elle ne vous donnera que le relatif.» Il faut séparer la raison de l'expérience; l'absolu est «en dehors de l'expérience et des conséquences qu'en tire la logique. Vous dites: 'après que l'intelligence a constaté le réel, la raison construit l'idéal'. Il faut s'entendre là-dessus. Cette construction, la raison ne peut l'élever qu'en partant de certains postulatums, qui sont en dehors de l'expérience. $\gg^{41}$

Certes, les explications prennent pour point de départ quelque chose d'inexpliqué, cependant celui-ci ne doit pas être en dehors de l'expérience. Les principes de la logique sont vrais «seulement parce qu'ils nous conduisent à des résultats que vérifie l'expérience. On pose un postulatum, on en déduit des conséquences, si ces conséquences sont vérifiées par l'expérience, le postulatum est admis, au moins provisoirement; si l'expérience ne vérifie pas les conséquences, le postulatum est rejeté. [...] En suivant ce principe, d'innombrables phénomènes de l'esprit se trouvent correspondre à d'innombrables phénomènes révélés par l'expérience. Cette correspondance cesserait entièrement, si on n'admettait pas ce principe.»

Avec des postulats douteux on peut faire des démonstrations, mais elles restent absolument incompréhensibles puisque leur vérification empirique est irréalisable. Par exemple, il est aisé d'être d'accord sur le postulat qu'il faut admettre comme semblables les êtres qui sont semblables. Mais peut-on dire qu'il est pareil à celui d'Euclide et que l'expérience le vérifie «dans le sens que tous les hommes le suivent»? La réponse est négative. «Quand j'admets le postulatum éthique en question, je sors de ce domaine [de l'expérience]; c'est donc seulement par un acte de foi que je puis l'admettre. Quand j'ai parlé de vérité dans le domaine de l'expérience, je n'ai pas voulu nier qu'il existât une

${ }^{41}$ V. Pareto, Euvres complètes. Tome XXX, op. cit., 334-337 et Matériaux..., op. cit., 123-125. Dans une lettre du 17 mai 1897 Pareto écrit à son ami Pantaleoni:«J'ai dit clairement à Naville que je suis positiviste, c'est-à-dire que rien n'existe pour moi en dehors de l'expérience. Ce n'est pas que je ne reconnaisse l'existence de l'inconnaissable, je la proclame même très haut, mais je n'admets pas que l'inconnaissable envahisse le domaine du connaissable. Il est donc libre de faire l'éthique qu'il voudra, mais qu'il avertisse d'abord son lecteur des postulats dont il a besoin. Les accepter ou les refuser est affaire de foi, non de science. Celui qui les accepte ira de l'avant, celui qui ne les repousse laissera s'arrêter même les conséquences. Sur ce chapitre il me semble que nous nous entendons, mais il ne veut pas admettre que tout le connaissable provient de l'expérience. Il croit que les axiomes sont vrais parce que le contraire est inconcevable ! Dire que je m'imaginais que plus personne n'avait des idées pareilles ! Mais maintenant je me tiens tranquille. Il m'importait seulement de montrer où nous nous séparons.». La lettre est publiée intégralement in V. Pareto, Euvres complètes. XXVIII/2. Lettere a Maffeo Pantaleoni, 1890-1925, a cura di G. De Rosa, Genève, Droz, 1984, pp. 72-75. 
vérité en dehors de ce domaine, mais évidemment ce second genre de vérités diffère essentiellement du premier.»

Même en acceptant comme acte de foi qu'il faut admettre comme semblables les êtres qui sont semblables, une doctrine éthique rationnelle doit recourir à plusieurs autres postulats, à des énoncés précis et vérifiables, pour s'approcher de la réalité empirique. Il n'en reste pas moins qu' «Une grande difficulté provient de ce que les hommes ne sont pas identiques et que par conséquent on ne peut admettre dans toute sa rigueur la formule: traite ton prochain comme toi-même. D'autre part, si vous concédez qu'une différence entre les hommes justifie une différence de leurs actions, il vous faut mettre une limite précise à cette concession, sinon vous détruirez complètement votre formule. Pour trouver cette limite, vous aurez certainement besoin d'un autre postulatum.»

On peut construire, en vérité, une doctrine éthique, s'en servir pour en tirer certains préceptes pour les actions sociales des hommes, mais il faut avertir préalablement le lecteur: «Voici l'éthique que j'adopte. Vous acceptez, ou vous n'acceptez pas ses postulata. Si vous ne les acceptez pas, brisons là, mon livre n'est pas pour vous. Si vous les acceptez et si mon raisonnement est correct, il vous faut admettre les conséquences auxquelles j'arrive.» Somme toute, Pareto concède à Naville qu'une partie de la science sociale doit être basée sur l'éthique, cependant il voudrait que son correspondant reconnaisse qu'il y a une autre partie de cette science dont l'objet est la description des manifestations de l'activité sociale et des variations sociales correspondant aux variations des états moraux, religieux, psychologiques des individus ${ }^{42}$.

L'essai de Naville sur l'économie dite politique et les sciences morales suscite également la réaction d'un autre économiste, Maffeo Pantaleoni. Celui-ci confirme au philosophe genevois son opposition à l'assimilation de l'économique à la mécanique rationnelle. «En science économique la force motrice est une cause finale, le désir de satisfaire un besoin avec un minimum de dépense. En mécanique, il n'y a pas de causes finales. En économique, les problèmes de collocation, d'énergie, de position, ou potentielle, sont très nombreux et vous aurez plus facilement des analogies dans la théorie de l'électricité que dans la mécanique rationnelle; mieux encore en physiologie.» Le même Pantaleoni juge formelle la conception de la morale comme théorie de la valeur des buts. Elle lui semble dissimuler une approche intuitionniste selon laquelle la conscience possède la faculté intuitive de classifier les motifs par rangs. En même temps la propension de Naville à réduire la morale à une série de principes de conduite, supérieurs à tous les autres et reconnus tels intuitivement par la conscience, lui semble périlleuse ${ }^{43}$.

Le philosophe genevois proclame que l'homme ne peut vouloir des choses qu'il sait impossibles, que l'impossibilité clairement perçue arrête la volonté et surtout qu'il est absurde de présumer qu'un acteur opte toujours pour le meilleur ${ }^{44}$. A quoi l'économiste italien réplique: «L'homme, de sa nature, aime la routine, il

${ }^{42}$ V. Pareto, Euvres complètes, op. cit., XIX, pp. 337-340 et Matériaux..., op. cit., pp. 126-129.

${ }^{43}$ Matériaux..., op. cit., pp. 129-131. Les citations se trouvent dans une lettre datée de Naples, le 13 mai 1897.

${ }^{44}$ La question de la liberté, «La Suisse», 27 avril 1898. 
ne change ses habitudes que quand il est contraint. La concurrence le met dans la dure nécessité de transformer ses habitudes ou de se ruiner. Soustrayez-le à cette contrainte ou à toute autre semblable, et vous pouvez bien être sûr qu'il changer le moins possibles ses chères habitudes. $\rangle^{45}$

Naville revient, dans un article paru au mois de Novembre, sur la question. Il affirme que ses amis économistes ont tort de réduire le syllogisme exclusivement au rôle de procédé de démonstration. La logique moderne, en fondant le raisonnement sur les critères de substitution et de non substitution, a montré que la conclusion d'un syllogisme peut inclure quelque chose de nouveau et de certain et que cela n'affecte pas son caractère scientifique. Pour cette raison le syllogisme reste, malgré le dépassement de la logique aristotélicienne, «un instrument de recherche, de progrès et d'acquisition ${ }^{46}$. Par ailleurs, il ne faut pas oublier que tous les objets des sciences sont abstraits. C'est par la volition réfléchie que le chercheur détache certains caractères du tout pour ensuite les analyser, en soi et pour soi.

Si l'ordre des phénomènes est donné par des lois générales, la distinction entre la science pure et la science appliquée est sans fondements. En effet, dans ce cas, les sciences appliquées demeurent étrangères aux sciences des lois. Disciplines historiques, elles s'efforcent de comprendre et de reproduire des objets complexes, uniques, donnés, en les construisant et en les reconstruisant ${ }^{47}$.

Après la lecture de ce texte, la réaction de Pareto, à la mi-décembre, est sans ménagements. «Vous mettez sur le même rang le théorème du carré de l'hypoténuse et une proposition qui dit que 'Si dans un Etat il n'y avait aucune croyance commune à un groupe social important...' et cette autre 'Les progrès du scepticisme ont contribué...'. La première est une proposition entièrement d'accord avec les faits et, partant, incontestable; les autres expriment des opinions très respectables, mais qui sont fort loin d'être démontrées.» «Le théorème du carré de l'hypoténuse a une probabilité extrêmement grande en sa faveur, parce qu'il s'accorde avec une immense quantité de faits. Les deux autres propositions que vous citez ont une assez petite probabilité en leur faveur: $1^{\circ}$ parce qu'elle ne sont guère précises. Qu'est-ce précisément qu'une croyance commune à un groupe social important? $2^{\circ}$ parce que les faits que vous pouvez citer comme s'accordant avec ces propositions sont aussi susceptibles d'une autre interprétation. En réalité, ces propositions expriment plutôt un état d'âme de plusieurs personnes qui désireraient qu'elles fussent vraies. Notez que je ne dis pas qu'elles sont fausses; mais c'est aux personnes qui les énoncent à les prouver. Vous avez bien raison de dire que les lois n'expliquent pas les faits. Mais les lois ne sont autre chose qu'une concordance des faits, elles expriment simplement une uniformité. Ce sont les métaphysiciens qui veulent leur donner le caractère de nécessité. Le type des lois naturelles est simplement celui-ci: le phénomène $A$ accompagne le phénomène $B$. En d'autre termes: $A$ et $B$ se rencontrent toujours ensemble. Bien entendu dans les

${ }^{45}$ V. Pareto, Euvres complètes, op.cit., XIX, pp. 338-389 et Matériaux..., op. cit., pp 143-144.

${ }^{46}$ La nouveauté dans la conclusion. Etude syllogistique, «Revue philosophique de la France et de l'Etranger», XXIV, 1899, II, pp. 263-270.

${ }^{47}$ Pour l' histoire. A propos de MM. Goblot et Milhaud, «Revue de Métaphysique et de Morale», VII, 1899, pp. 751-758. 
limites de notre expérience, car nous ne connaissons rien au-delà. Expliquer un fait, c'est uniquement le mettre en rapport avec d'autres faits.» ${ }^{48}$

Naville prend conscience que Pareto veut se cantonner dans le domaine de la science de la société, qu'il n'est nullement intéressé aux rapports entre les disciplines analytiques et les disciplines synthétiques, qu'il n'est pas concerné par des questions philosophiques ou du domaine de la métaphysique. Malgré cela, le professeur de Lausanne reste un lecteur attentif, perspicaces et diligent des travaux du penseur genevois.

\section{La réorganisation des enseignements des sciences sociales à Genève}

Doyen de la Faculté des Lettres de 1896 à 1902, Adrien Naville essaie de développer les enseignements des sciences sociales et politiques. En peu de temps trois licences (sciences sociales, économie politique et sciences politiques) sont mises sur pied et divers enseignants nommés aux chaires de Finances et Statistique, de Géographie, d'Histoire économique et sociale, d'Economie politique, tandis que ceux d'Histoire générale, de Sociologie et de Philosophie voient leur domaine d'enseignement agrandi ${ }^{49}$.

Naville attribue aux sciences sociales l'étude des relations sociales, des relations entre des êtres conscients et régies par des lois nécessaires et naturelles. Cette étude doit analyser la nature de ces relations dans le passé et dans le présent, mettre en évidence, parmi les relations possibles, celles que l'on doit désirer, vouloir et s'efforcer d'institutionnaliser. La théorématique sociale, la science abstraite du social étudie les conditions et les circonstances dont dépendent tels résultats qui intéressent les relations sociales dans leur ensemble. L'histoire sociale étudie les relations sociales effectives, c'est-à-dire les faits à la base du présent, sans la connaissance desquels on ne peut pas prévoir ou préparer l'avenir. Il répute que l'historicisme et le pseudo historicisme de la philosophie allemande, le foisonnement de la recherche historique du $\mathrm{XIX}^{\mathrm{e}}$ et des débuts du $\mathrm{XX}^{\mathrm{e}}$ siècles, ont ébranlé la croyance dans l'unité de l'esprit et favorisé la reconstruction et l'hégémonie d'un système amphibologique de la raison pratique. La conscience morale n'est pas le produit mobile des circonstances, du milieu, de la race, de l'éducation, de la nature individuelle; elle est la source des jugements sur la valeur comparée des buts vers lesquels il faut aspirer. Si le Créateur a gravé dans l'âme de l'homme certains principes d'amour et de justice, c'est à l'homme lui-même de les conserver et de les pratiquer ${ }^{50}$. Enfin, à la canonique sociale ou science des programmes pratiques est assigné la mission d'étudier comment obtenir une organisation sociale harmonieuse, quel devrait être le rôle de l'Etat et de l'autorité, les rapports des individus avec les institutions juridiques et politiques.

Dans cette perspective la sociologie doit mettre en lumière les conditions générales et les processus rendant possible la production et la reproduction de la

${ }^{48}$ V. Pareto, Euvres complètes, op. cit., XIX, pp. 396-397 et Matériaux..., op. cit., pp. 147-148.

${ }^{49}$ A. Naville, Université de Genève. Faculté des lettres et des sciences sociales, 1895-1914, Genève, Georg, 1914; Matériaux..., op. cit., passim; M. Marcacci, Histoire de l'Université de Genève, 1559-1086. Préface de J. Thorens, Genève, Université, 1987.

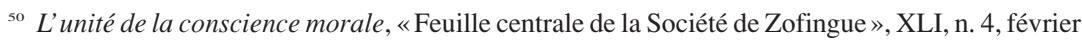
1901, pp. 251-261 et en tiré à part: Genève, Impr. Journal de Genève, 1901, 14 p. 
société humaine; elle doit également montrer l'interdépendance des relations sociales, les liaisons nécessaires entre les causes et les effets. En somme, elle est chargée d'élaborer les connaissances de base pour évaluer et utiliser les moyens indispensables à l'obtention de buts désirables ${ }^{51}$.

A la suite de la non réélection de Naville au Décanat de la Faculté, ce programme, critiqué par des professeurs pour lesquels le passé n'était pas un bien présent mais plutôt un bien futur, ne sera pas réalisé intégralement. Pantaleoni écrira: «J'avais cru que tant que Genève conserverait sa cathédrale de Saint-Pierre, la faculté conserverait son doyen. Qui doit être bien aise de cette révolution de palais, c'est Madame Naville, à la perspicacité de laquelle les petites conspirations qui de temps en temps se nouaient, n'échappaient pas et qui en souffrait, comme de raison. $»^{52}$

Surtout l'évolution de l'enseignement de la sociologie, «confié à un homme préoccupé surtout du côté économique des questions sociales », afflige tout particulièrement Naville. «Nul doute que le facteur économique n'ait dans la vie des sociétés et jusque dans leur organisation politique un rôle très important, nul doute même, pour moi tout au moins, que l'importance de ce facteur n'ait été comprise que d'une manière insuffisante par la plupart des historiens. Mais ce facteur n'est pourtant pas le facteur unique, tant s'en faut. Il y en a d'autres tout aussi importants. Dans les publications sociologiques les plus récentes la plus grande place n'appartient nullement à l'économique. Elle appartient bien plutôt à l'histoire religieuse et à celle des idées de tout genre, des mœurs, des institutions, des arts, du langage, de la poésie. [...] Séparer la sociologie de la philosophie morale et sociale, de la psychologie, de la linguistique, de l'histoire des sociétés envisagées sous tous ses aspects et dans tous ses facteurs, ce serait la priver de son atmosphère et la museler. Ceux qui s'intéressent au développement de cette jeune science seraient bien mal avisés s'ils lui coupaient les ailes et prétendaient l'enfermer dans un domaine trop étroit.. ${ }^{53}$

Pantaleoni intervient pour éviter que le partage des matières entre les disciplines ne soit pas un simple jeu de mots. Par exemple, il considère que la méthodologie statistique appartient au professeur de logique. «La statistique n'est pas spéciale aux faits économiques et sa méthode est générale pour une catégorie de faits. En outre, les étudiants n'y comprennent rien s'ils ne placent pas la méthode statistique dans le système des méthodes logique.» Il n'est pas d'accord non plus de réserver l'étude des faits à l'histoire car tous les cours théoriques font appel aux faits. Les cours de droit lui paraissent essentiels. Ils évitent aux étudiants de tomber dans la «superficialité», le «charlatanisme», le «faux semblant», le «journalisme» et les obligent à comprendre un système concret et à l'étudier dans son histoire, dans les processus de formation. Pour l'économiste Pantaleoni les curricula doivent être souples et les filières perméables. «Observez et essayez et réformez. Tout est trop nouveau pour une cristallisation. ${ }^{54}$

${ }^{51}$ A. Naville, Programme sommaire des sciences sociales. Etude dédiée à l' Université de Genève à l'occasion du Jubilé de 1909, Genève, Georg, 1909.

${ }^{52}$ Lettre du $1^{\text {er }}$ août 1902, in Matérieaux..., op. cit., pp. 197-201.

${ }_{53}$ A. Naville, Université de Genève..., op. cit., pp. 20-24.

${ }^{54}$ Lettre du 26 avril 1901 in Matériaux..., op. cit., pp. 165-173. Cf. L. Michelini, Il pensiero di Maffeo Pantaleoni tra economia politica e politica militante, «Società e Storia», n. 58, 1992, pp. 721 787 et du même Marginalismo e Socialismo : Maffeo Pantaleoni (1882-1904), Milano, Franco Angeli, 1998. 
D'un avis opposé est un autre économiste, Léon Winiarski, pour lequel essentielle et indispensable reste la distinction entre la partie rationnelle et abstraite des phénomènes et la partie appliquée qui étudie leur forme concrète et détaillée. La construction rationnelle et abstraite (l'étude des rapports entre les concepts) joue en rôle plus important en économie, en sociologie et en psychologie que la partie historique et descriptive basée principalement sur l'observation. Ces constructions constituent les fondements d'une nouvelle science, la mécanique sociale, «véritable science des lois sociales, servant de fondement pour les lois juridiques. Et c'est ainsi que la justice sociale, sous forme de légalité contractuelle, tendra de plus en plus à se confondre avec la vérité sociale synthétique. Et toute cette évolution est dirigée par un principe économique et notamment par le principe du moindre effort.» ${ }^{55}$

L'intérêt de Naville pour son Université restera constant au fil des années. Il interviendra à l'occasion de la création de nouveaux enseignements et de nominations professorales et toujours avec le souci de relier les cas concrets à des principes théoriques généraux ${ }^{56}$.

\section{En quête d'une philosophie des sciences sociales}

Au cours de la première décennie du $X X X^{\mathrm{e}}$ siècle Naville va approfondir beaucoup de sujets déjà traités mais critiqués, ici et là, en son temps, et en aborder d'autres retenus cruciaux, essentiels pour mieux systématiser, en vue d'une nouvelle édition, la présentation de sa classification des sciences.

Tout d'abord il s'évertue à démontrer que la vérité est l'accord de la pensée avec des objets, qu'elle est donc une caractéristique de la pensée. Par contre, un fait est ou n'est pas, il est réel ou pas. L'accord de la pensée avec des objets n'est jamais absolu d'où la relativité de la connaissance scientifique. La vérité est donc un idéal, pour s'y approcher le sujet doit purifier «sa raison pratique de toutes les influences étrangères qui la corrompent $»^{57}$.

Pendant cette décennie Naville s'intéresse beaucoup à la psychologie conçue comme la science des lois de la vie psychique. Il énumère les difficultés que cette discipline rencontre dans l'établissement de ses théorèmes, mais aussi la solidité de certaines de ses approximations. Les événements psychiques conscients sont des faits et ils apparaissent tels quels à l'observateur attentif et prudent. La connaissance psychologique est directe et immédiate, par la raison elle aspire à l'universel, ses perceptions de la pensée, des sentiments, de la volonté sont spéciales, pas affectées par les apparences constatées; par contre, dans la réalité physique, par l'effet de l'aperception, difficilement contrôlable, de l'observateur, les apparences parasitent la connaissance.

La loi, en psychologie, étant un rapport entre deux possibilités, n'exclue pas la liberté. Pour cette raison le déterminisme est défini une fausse doctrine croyant

${ }^{55}$ Les interventions datent de 1900 et de 1904 et se trouvent à présent in L. Winiarski, Essais sur la mécanique sociale. Textes réunis et présentés par G. Busino, Genève, Droz, 1967, pp. 291-306.

${ }^{56}$ La législation ouvrière à l' Université, «Journal de Genève», 28 février 1912 et La loi universitaire, Ibid., 8 décembre 1912.

${ }^{57}$ De la vérité. Remarques logiques, «Revue philosophique de la France et de l'Etranger», XXIX, 1904, I, pp. 449-461. 
que le présent est contenu dans le passé, que l'avenir est déjà dans le présent, que l'initiative, l'évolution créatrice et la destruction ne sont que des formules vides.

Naville admet qu'il est difficile de distinguer les sentiments des opinions, qu'une théorie de l'amour-propre entraîne des équivoques et des doubles sens, et néanmoins il s'efforce de comprendre les raisons nombreuses et diverses donnant à l'opinion, aux sentiments et à l'amour propre la place qu'ils ont dans les relations sociales. Il distingue dans les perceptions données par les sens celles de la vue et de l'ouïe, placées à la source des plaisirs esthétiques. Les perceptions des autres sens n'appartiennent pas au domaine esthétique alors que les idées de ces perceptions lui appartiennent. La science, la philosophie, la politique, la morale, ne sont pas des créations esthétiques, mais elles peuvent être à l'origine des œuvres d'art, dont le domaine n'a d'autre limite que celle de l'esprit humain ${ }^{58}$. La théorie du jugement psychologique, de la négation comme une relation négative entre des représentations ainsi que les débats sur les rapports entre le jugement esthétique et le jugement historique, lui paraissent des sophismes verbaux et il les critique sans précautions rhétoriques ${ }^{59}$.

Naville s'interroge également sur les apports de la linguistique générale à la connaissance. La lecture d'un livre de Ch. A. Séchehaye lui fait dire que la linguistique théorique est une science qui cherche des principes d'explication, des lois universelles, et pas du tout une science des faits localisés dans le temps et dans l'espace ${ }^{60}$.

La certitude dans l'existence de lois universelle lui fait écrire des pages d'une extrême sévérité contre le pragmatisme. Absurde lui parait la réduction de la science à un instrument pratique, à un ensemble de conventions et d'artifices à l'aide desquels nous nous adaptons au monde et grâce auxquels nous ajustons le monde à nous. Inacceptable lui parait la thèse que la vérité scientifique est un moyen au service de l'action. Cette «scholastique protestante»n'admet aucune évidence immédiate, nie la raison pratique aussi bien que la raison théorique, proclame que le vrai est ce qu'il est utile de croire tel. La défiance à l'égard de la spéculation métaphysique, de la science objective, de la connaissance impersonnelle, désintéressée, oblige la philosophie pragmatiste à valoriser uniquement les conséquences des actions scientifiques sur la vie des êtres humaines et des collectivités. Une telle «croyance » est irréaliste, irréfléchie, contraire au savoir courant et au sens commun ${ }^{61}$. Un jugement analogue est porté sur la philosophie de Bergson, sur son procès à la science, sur ses critiques à la construction scientifique accusée de recourir à une fausse représentation du temps, de négliger les pouvoir de l'intuition, de postuler l'existence de deux vérités: la vérité philosophique impliquerait la liberté et la scientifique serait caractérisée par le déterminisme.

${ }^{58}$ Linéaments de psychologie esthétique, «Archives de psychologie», II, 1903, n. 6, pp. 89-105.

${ }_{59}$ Naville s'en explique dans le compte rendu du livre de Ernest Schrader, Zur Grundlegung der Psychologie des Urteils, «Archives de psychologie», III, n.4, 1904, p. 330. Voir aussi C. Scognamiglio, Giudizio storico e giudizio estetico in un dibattito di fine Ottocento, «L'Acropoli», V, n. 5, settembre 2004, pp. 541-556.

${ }^{60}$ Le programme et les méthodes de la linguistique théorique. A propos d'un ouvrage récent, «Revue de synthèse historique», XVI, 1908, pp. 175-181.

${ }^{61}$ Anti-Pragmatisme, «La Semaine littéraire», XVII, n. 792, 6 mars 1909, pp. 109-111. 
Naville écrit à ce propos: «Ma conclusion au sujet du procès de la science et de la théorie des deux vérités est donc que la science est limitée, relative, partiellement conventionnelle, qu'elle baigne dans le mystère et laisse ouvert un monde de questions qui relèvent de la spéculation transcendante, mais que, dans son domaine et là où elle se prononce, il n'y a pas d'autorité supérieure à la sienne.» ${ }^{62}$

L'autre thème travaillé longuement est celui de la nature de la sociologie en tant que science des lois naturelles, conditionnelles et universelles, des possibilités sociales, des relations entre les hommes, des rapports de dépendances nécessaires, échappant aux appréciations morales ${ }^{63}$.

A l'opposé d'Emile Durkheim pour lequel les faits sociaux ou les relations sociales, sont extérieurs, agissent du dehors sur les individus et exercent une contrainte sur eux, Naville revendique l'antériorité du psychique des individus. Les habitudes, les rapports fréquentatifs, les comportements répétitifs, augmentent l'importance des relations interhumaines. Il caractérise la relation sociale comme un événement (matériel, organique ou inorganique) par lequel des êtres conscients exercent ou subissent des influences les uns à l'égard des autres. La relation sociale n'est pas la société, elle est plutôt le moyen grâce auquel les individus, «les âmes conscientes individuelles» se réalisent. Elle est la résultante de trois espèces différentes de causes (psychiques, somatiques et techniques), et prend les formes de la contrainte (corporelle et morale), de la collaboration, de l'échange, de la donation, de la spoliation, de l'autorité (commandement et obéissance), de l'intériorisation du système des signes et du langage.

La sociologie est la science des relations sociales. Elle explique pourquoi et comment ces relations se transforment, deviennent caduques, resurgissent, disparaissent, ou en engendrent des nouvelles sous l'impulsion d'événements psychologiques, biologiques, physiques, ou «d'autres choses». A une telle science appartient la tâche de discerner la puissance relative des causes dans l'enchevêtrement et la complexité de la vie sociale, d'élaborer les lois générales des relations interhumaines et de déterminer les conditions de leur production, de leurs durée, modification et suppression. Les jugements de la sociologie sont différents des jugements de l'histoire. Les premiers sont conditionnels tandis que les seconds sont catégoriques et inconditionnels. Les lois de la sociologie ont le caractère de rapports conditionnels, elles indiquent la dépendance de certains résultats à l'égard de conditions possibles. Ses énoncés sont des affirmations conditionnelles, introduites par un si, et ses prévisions se fondent sur des lois nécessaires appliquées aux conditions contingentes que l'observation constate dans le présent. Par contre, il n'y a pas de lois historiques, mais des rapports généraux, des uniformités non universelles. L'histoire élabore des jugements catégo-

${ }^{62}$ Le procès de la science et la théorie des deux vérités. Remarques sur le rapport entre la philosophie et les sciences, «Revue de théologie et de philosophie», III, n. 16, septembre-octobre 1915, pp. 237-253. Dans une lettre du 22 décembre 1915, V. Pareto, Euvres complètes, op. cit., XIX, p. 909 et Matériaux..., op. cit., p. 226, dit son accord sur l'impossibilité d'admettre a priori le déterminisme. Il avance néanmoins la réserve que dans maints cas les phénomènes sociaux paraissent déterminés par certaines conditions et que la science n'a rien à dire et ne peut rien connaître en dehors de l'expérience.

${ }^{63}$ La sociologie abstraite et ses divisions, «Revue philosophique de la France et de l'Etranger », XXXI, 1906, I, pp. 457-471 et «Bulletin de l'Institut national genevois », XXXVI, 1907, pp. 335-356. Il existe une version brochure de cet essai, Genève, Impr. Centrale, 1906, 24 p. 
riques, basés sur des rapports inconditionnels, sur des faits nécessaires ${ }^{64}$. Ces jugements ne peuvent prétendre qu'à une universalité fermée. L'universalité ouverte appartient surtout aux jugements conditionnels, c'est-à-dire à des jugements qui affirment conditionnellement des possibilités de relations nécessaires. La conditionnalité des résultats «n'affranchit pas l'homme que s'il croit au pouvoir de la volonté libre d'intervenir dans la production de l'avenir, en posant des conditions dont elle est elle-même créatrice ${ }^{65}$.

Mais si nous ne pouvons pas formuler des assertions ayant un caractère absolu, comment alors différencier les jugements conditionnels des jugements hypothétiques?

Le 23 mai 1905 Pareto écrit à Naville et lui fait observer qu'en science il n'y a pas de relations nécessaires. «Toute chose n'étant connu, expérimentalement et nécessairement, que dans certaines limites de temps et d'espace, ce qui est nécessaire (ou mieux: nous paraît tel) dans ces limites peut ne pas l'être dans d'autres limites.» Il n'y a pas de jugements scientifiques ayant le caractère d'universalité ouverte, «car un tel jugement affirmerait quelque chose en dehors des limites de temps et d'espace qui bornent nos connaissances ». La distinction entre la science des lois et la science des faits est superfétatoire. Toute science, quelle qu'elle soit connaît ses limites et ne va pas au-delà du relativisme scientifique ${ }^{66}$.

La question de l'équivalence entre les jugements conditionnels et les jugements hypothétiques est abordée aussi par Maffeo Pantaleoni dans la lettre du 22 septembre $1906^{67}$. Cet économiste dit de ne pas comprendre le sens de la phrase: la sociologie «cherche donc les lois en vertu desquelles telles ou telles relations entre les hommes dépendent d'autre chose, sont modifiées, augmentées, diminuées, ou supprimées par autre chose.» Faut-il entendre des choses hypothétiques en plus des choses réelles (celles-ci possibles historiquement, réelles, basées sur l'expérience)? Or les choses hypothétiques sont innombrables et, dans la plupart des cas, dépourvues de buts pratiques, généralement irréalisables et impraticables. Abandonner l'expérience et l'induction dans l'élaboration des hypothèses exposerait le chercheur à des risques innombrables. Les hypothèses doivent être des abstractions de réalités complexes, sans contradictions, en accord avec la base inductive, avec la réalité. «Les hommes ont eu, par exemple, les goûts les plus différents: mais cette variation a toujours eu des limites, des bornes, et je ne saurais supposer des goûts qu'aucune induction justifierait. Je ne saurais supposer une société de paranoïaques, parce que les types paranoïaques n'ont jamais duré assez pour me fournir une société. En un mot, comment dois-je vous comprendre lorsqu'il s'agit des sciences théorématiques sociales? L'infini nombre d'hypothèses possibles concernant leurs conditions d'existence ne doit-il pas être borné par l'intuition?»

${ }^{64}$ La notion de loi historique, «Revue de synthèse historique», IX, juillet-décembre1904, pp. 16. Ce texte a paru également dans le volume: Congrès international de philosophie. IIme session tenue à Genève du 4 au 8 septembre 1904. Rapports et comptes rendus publiés par les soins du Dr Ed. Claparède, Genève, Kündig, 1904, pp. 680-687.

${ }_{65}^{6}$ La primauté logique des jugements conditionnels, «Revue philosophique de la France et de l'Etranger », XXX, 1905, I, pp. 337-345.

${ }^{66}$ V. Pareto, Euvres complètes, op. cit., XIX, pp. 545-546 et Matériaux..., op. cit., pp. 214-215.

${ }^{67}$ Matériaux..., op. cit., pp. 216-217. 
Pour Naville ces remarques sont dictées par une incompréhension de fond de sa doctrine sur la diversité des objets, sur la métaphysique et sur la logique, sur l'impuissance de la logique à faire progresser la philosophie. Pour la métaphysique la raison est une réalité permanente et suprême tandis que pour la logique elle est un idéal, une règle souveraine pour toutes les sciences, soit pour celles de la réalité que pour celles de la possibilité. La logique confond l'identité avec l'égalité, le conditionnel avec l'hypothétique; elle fait de la contradiction un rapport objectif alors qu'il s'agit d'un rapport subjectif, intellectuel, psychologique. Il est erroné de confondre la contradiction avec l'opposition. Cette dernière consiste en un rapport entre deux jugements de mêmes qualité et identité mais dont les sujets ou les prédicats sont à tel point différents qu'on les considère comme opposés. Or les prédicats différents permettent des jugements affirmatifs tandis que la logique de l'identité interdit l'opposition et condamne la contradiction.

Naville répète qu'il y a une seule logique, celle de l'identité mais dont l'application intégrale est restreinte. Cette logique de l'identité peut donner des réponses approximatives et «tout progrès dans l'approximation est une conquête féconde». Elle peut faire la théorie de la vérité partielle, de la connaissance approximative, de la marche ascendante de l'esprit dans l'approximation, mais elle ne pourra jamais remplacer le savoir métaphysique ${ }^{68}$.

\section{La classification des sciences}

La question de la classification des sciences débute avec Aristote. Depuis, au fil des siècles et jusqu'à Auguste Comte, on a essayé de mettre de l'ordre dans le foisonnement des disciplines. Cette problématique a toujours attiré l'attention des milieux intellectuels genevois. Candolle, dans son introduction de la Théorie élémentaire de la botanique (1813, 1819, 1844), pour justifier le statut scientifique de la botanique, proposa une classification générale des sciences: rationnelles, testimoniales ou historiques et expérimentales ${ }^{69}$.

Ernest Naville, on le sait, accepte la doctrine de Maine de Biran, son idée qu'il y a autant de sciences que des points de vue, que chaque science détermine un ordre de faits, déploie une méthode propre, atteint des résultats spécifiques et rencontre des obstacles particuliers. Par conséquent, il considère que la classification des sciences est la tâche fondamentale en vue de déterminer la place respective de chacune d'elle dans la hiérarchie de la connaissance et de connaître leurs apports, leurs emprunts, leurs échanges avec la philosophie et la religion ${ }^{70}$. Son

${ }^{68}$ Logique et mathématique. Identité, égalité, article refusé par la rédaction de la «Revue de Métaphysique et de Morale» en janvier 1913 (le manuscrit autographe appartient au professeur Gabriel Aubert) et La logique de l' identité et celle de la contradiction. Notes critiques, Genève, Georg, 1909 (Mémoire publié à l'occasion du Jubilé de l'Université par les soins de la Commission des publications. Fonds du Jubilé).

${ }^{69}$ Voir à ce propos J.-M.Drouin, Classification des sciences et classification des plantes chez Augustin-Pyramus de Candolle, «Revue de synthèse », tome CXV, nos 1-2, janvier-juin 1994, pp. 149165 .

${ }_{70}$ Pour en savoir davantage v. M. Manganelli, Il pensiero di Ernest Naville, Milano, Marzorati, 1969-1973, 2 vols.; F. Azouvi, Science de l'homme et division des sciences selon Maine de Biran, «Revue de synthèse », tome CXV, nos 1-2, janvier-juin 1994, pp. 55-69 et l'essai de Dario Antiseri, $L a$ 
fils Adrien poursuit les investigations que le père avait résumées dans l'essai de 1885 intitulé Les système de philosophie, mais en plus il réfléchit sur la philosophie du droit d'Ernest Roguin, sur les travaux de Heinrich Rickert, sur sa doctrine de la «conceptualisation individualisante» et de l'activité du sujet par rapport à des valeurs, et puis il élabore des ébauches de classification; il en publiera un premier aperçu en 1888.

La science est le produit du libre effort de l'homme; elle ne peut pas se passer des règles idéales pour ses activités et sa pratique. Le travail du chercheur est gouverné par la liberté. Les sciences son réparties en trois groupes.( a) les sciences du réel ou sciences des êtres (histoire); (b) les sciences des conditions nécessaires du possible, ou sciences des lois (théorématiques); (c) les sciences de l'idéal ou des règles de l'activité (sciences régulatrices).

L'histoire (a) donne les récits, les descriptions, les explications, fort incomplètes, d'un nombre limité d'événements et des changements; la théorématique (b) énonce des rapports et des lois caractérisant la physique, la chimie, la biologie, la psychologie et la sociologie, tandis que les sciences régulatrices (c) fixent les jugements de valeur et donnent les règles, les préceptes et les modèles normatifs des conduites ${ }^{71}$.

Plus qu'une classification Naville élabore un classement, une mise en correspondance de ce qui est classé avec un ordre préalablement donné. La préoccupation d'ordre pratique est évidente. Dans chaque catégorie il y a un même nombre de numéros. En face de chaque science des lois il y a une science historique spéciale. Par exemple, la statistique se trouve en histoire mais placée en face de l'arithmologie appartenant à la théorématique.

Dix ans plus tard, ayant changé d'avis su un certain nombre de questions, et notamment qu'en histoire il n'y a pas de divisions car cette discipline étudie le concret et que dans le concret tout agit sur tout, Naville publie une nouvelle esquisse de classification. Les instruments conceptuels utilisés ne sont pas formalisés, d'ailleurs ils n'ont pas besoin de formalisation. Grâce à la distinction entre la loi et la règle, la logique n'est qu'une discipline normative, canonique, un ensemble de règles pour servir une théorie de la valeur. La mise en ordre intellectuelle est basée sur un ordre particulier, constitué à la fois par des caractères essentiels spécifiques, par l'existence d'une structure semblable et d'une origine commune $^{72}$. Il s'agit d'une fiction postulant la réalité de natures permanentes et communes et donc la connaissance reste approximative. Par moment, cette construction intellectuelle parait s'inspirer de celles de A. A. Cournot, de H. Spencer et de A. Comte, quoique d'une façon plus subtile et nuancée, mais en réalité elle est très différente car le rejet de la position ontologique et du déterminisme est opéré avec le but de donner un rang prééminent à la spéculation trans-

logica dell' ipotesi di Ernesto Naville e l' ipotetismo tra Ottocento e Novecento, in E. Naville, La logica dell' ipotesi. Traduzione di A. Fabriani. Introduzione di D. Antiseri, Milano, Rusconi, 1989, pp. 7-102. On peut également lire cet essai in D. Antiseri, Ragioni della razionalità. Interpretazioni storiografiche, Soveria Mannelli, Rubbettino, 2005, pp. 173-287.

${ }^{71}$ De la classification des sciences. Thèses et questions. Etude logique, «La critique philosophique», 1887, 3(2), pp. 321-342 et pp. 401-415, texte repris en brochure, Genève, Georg, 1888, 47 p.

${ }^{72}$ Le principe général de la classification des sciences, «Archiv für systematische Philosophie», Bd. IV, 1898, Heft 3, pp. 364-381. 
cendante. A ce propos Naville écrit, dans le volume de 1901, aux pp. 177-178: «La division du travail s'est établie dans le domaine intellectuel, comme dans celui de l'industrie, et les savants ont exclu de leur programme la spéculation philosophique. Mais ils se tromperaient s'ils croyaient avoir changé l'esprit humain, à ce point qu'il ait cessé de s'intéresser à ce qui dépasse la science proprement dite. Les termes du problème spéculatif se modifient en une certaine mesure avec le développement des sciences; le problème ne perd rien de son importance. Notre époque appelle un système spéculatif nouveau qui fonde ses affirmations transcendantes sur cette triple base: les natures permanentes et uniformes des choses, leur ordonnance progressive, l'autorité que s'attribue la raison pratique. Dans l'intérêt même de la science, et pour écarter une réaction qui l'emporterait, il importe de dire bien haut que si elle est un des trésors le plus précieux de l'humanité, elle n'est pas son seul trésor.»

Les questions auxquelles les sciences doivent répondre portent su ce qui est ou non possible, sur ce qui est réel ou irréel et sur ce qui est bon ou mauvais. De cela dérive leur groupement en trois grandes classements ou trois séries parallèles: les sciences du possible ou théorématiques (rapports possibles et conditionnellement nécessaires), les sciences du réel ou histoire (possibilités réalisées ou des faits) et les sciences du bon ou canoniques ou normatives (règles de l'activité humaine en vue de la réalisation du meilleur possible).

La nouveauté de cette classification consiste dans l'affirmation que les lois des sciences théorématiques ne portent pas sur les êtres et les événements mais sur des rapports conditionnels, de coexistence et/ou de succession, des rapports qui se produisent si certains termes sont posés. Ayant été réalisés une fois, ils pourront toujours se réaliser dans les mêmes conditions. La loi, dans sa valeur objective, en indique la nécessité. Les lois ne sont pas réelles. Si dans la situation de l'époque la psychologie et la sociologie ne sont pas classées parmi les sciences des lois, cela tient au fait qu'elles font appel à des généralisations historiques portant sur les caractères moyens des groupes donnés par l'expérience. Par exemple, l'homme moyen de Quételet ou la typologie des suicides de Durkheim.

Par contre, l'histoire est la science du réel, du possible réalisé et pas de ce qui n'a jamais été encore réalisé. Etant donné qu'elle n'est soumise à aucune conditionnalité, à aucun si, l'histoire élabore surtout des affirmations catégoriques.

Quant à la science du bon ou canonique, elle s'occupe de l'ensemble des règles générales de l'activité humaine, c'est-à-dire de la théorie générale des buts (la morale), des moyens (les arts), et des combinaisons des moyens pour atteindre les divers buts humaines (les sciences normatives).

L'accueil réservé à ces travaux pousse Naville à les refondre et à en faire une nouvelle édition ${ }^{73}$. Une décennie plus tard il va en faire paraître la troisième

${ }^{73}$ Nouvelle classification des sciences. Etude philosophique.Deuxième édition entièrement refondue, Paris, Alcan, 1901. Le sous-titre de la publication de 1888 était «Etude logique», remplacé maintenant par «Etude philosophique» car «La classification des sciences n'est pas de la logique pure, mais de la logique appliquée; elle trace les limites des sciences spéciales, distingue les notions fondamentales de chacune d'elles et marque les relations qui les unissent. Ainsi elle pose le seul fondement solide sur lequel puisse être élevée une construction spéculative» (pp. VI-VII). Ce livre a fait l'objet d'une réimpression anastatique à Paris, chez Didier Erudition, en 1991, avec une introduction (pp. I-IX) de Pierre Caussat. 
édition entièrement renouvelée ${ }^{74}$. La réception de l'œuvre s'est faite tantôt à partir du volume de 1901 et tantôt à partir de celui de 1920. Une étude de cette réception devrait en tenir compte ${ }^{75}$. Ici on se bornera à marquer les points forts des doctrines de Naville et à faire abstraction de leur formation, de leur évolution ainsi que des commentaires suscités à des moments divers et des critiques adressées à sa classification des sciences particulières.

L'idée centrale de ses doctrines reste le refus absolu de séparer les sciences de la philosophie et de la religion. Le classement des sciences, spécifié plus minutieusement, reste pareil à celui des travaux précédents. La théorématique comprend les sciences des limites universelles et des relations nécessaires des possibilités ou sciences des lois (la nomologie, les sciences mathématiques, les sciences physiques et les sciences psychologiques). L'histoire est la science des possibilités réalisées ou sciences des faits (histoire naturelle, histoire humaine). La canonique réunit les sciences des possibilités dont la réalisation serait bonne ou sciences des règles idéales d'action (théorie des moyens ou des arts, sciences morales ou théorie de la combinaison des moyens, morale ou théorie des buts obligatoires et de leur hiérarchie).

Pour classer les sciences qui naissent de l'observation de la diversité et qui cherchent à organiser cette diversité, Naville postule l'existence d'un ordre sousjacent. Cette notion le dispense de chercher une correspondance termes à termes entre les classes mais lui permet de mettre en place la confrontation entre l'extrême du groupe (faible) et de la classe (forte). L'élément conditionnel, étranger à l'histoire, caractérise non seulement les sciences physiques et naturelles mais également les sciences morales.

La distinction nette entre les lois et les faits, entre les lois et les règles ou les normes, a suscité beaucoup de réserves et de critiques. La séparation proposée a été jugée irréaliste. Si les faits déterminent la nature des lois, alors ils sont indissolublement liés réciproquement dans la réalité. Un fait peut être contingent à un moment donné mais à d'autres moments exprimer une liaison constante. Il a été observé aussi qu'il vaudrait mieux d'indiquer, dans chaque discipline, «l'aspect historique du phénomènes ou événements étudiés » car «la notion d'invariance fonctionnelle exclut également les cloisons étanches ${ }^{76}$.

Le lien établi entre la biologie et la psychologie, entre le système des signes («par lesquels les êtres associés font connaître les uns aux autres leurs sentiments,

${ }^{74}$ Classification des sciences. Les idées maîtresses des sciences et leurs rapports. Troisième édition, entièrement renouvelée, Paris, Alcan, 1920.

${ }^{75}$ Je cite ici uniquement les travaux dont cet écrit a tiré des profits: G. Vailati, Recensione, «Rivista di biologia generale», IV, vol. III, luglio 1902, et puis in G. Vailati, Scritti, a cura di M. Quaranta. I. Scritti di filosofia, Bologna, Forni, 1987, pp. 303-313; F. Enriques, La filosofia positiva e la classificazione delle scienze, «Scientia», IV, 1910, vol. 7, pp. 369-385; A. Reymond, Philosophie spiritualiste. Etudes et méditations. Recherches critiques, Lausanne, Rouge, 1942; J. Mouhasseb, Essai sur la classification des sciences, Damas, Impr.de 1'Université syrienne, 1953; J. Piaget, Classifications des disciplines et connexions interdisciplinaires, «Revue internationale des sciences sociales », XVI, 1964, n. 4, pp. 598-616; Classification des sciences et principaux courants épistémologiques contemporains, in Encyclopédie de la Pléiade, Logique et connaissance scientifique. Volume publié sous la direction de J. Piaget, Paris, Gallimard, 1967, pp. 1147-1224; Psychologie et épistémologie, Paris, Gonthier, 1970, pp. 149-187.

${ }^{76}$ A. Reymond, op. cit., I, p. 349. 
leurs pensées, leurs volontés ») et la sociologie en tant que science des contraintes sociales, relève de l'intuition créatrice. Que la sémiologie de Ferdinand de Saussure, science des lois de la création et de la transformation des signes, soit considérée une partie essentielle de la recherche sociologique, montre l'ampleur de l'ouverture de ce philosophe aux disciplines en train de se faire ${ }^{77}$.

La référence à de Saussure disparaît dans l'édition de 1920 de la Classification des sciences. Deux chapitres sont consacrés à l'analyse des distinctions entre les lois et les faits généraux, entre les lois naturelles et les essences métaphysiques, entre les lois et les règles, entre les lois de coexistence et de succession, entre la causalité naturelle et la causalité efficiente. Toutefois la tripartition de la matière ne change pas (théorématique, histoire, canonique). C'est la distribution à l'intérieur de chaque catégorie à subir des changements importants. En effet, dans la science des lois ou théorématique se trouvent désormais les mathématiques, la psychochimie, la biologie somatique, la psychologie et la sociologie ${ }^{78}$. Dans la science des faits, ou histoire, il est question des «objets historiques», des «lois historiques », des «possibilités historiques », de l'histoire humaine, de la géographie humaine et de la philologie. Enfin, dans la science des règles ou canonique, ont été placés la logique, la télèocanonique, la praxicanonique, le plaisir comme moyen et comme but, la classification des arts.

Pareto, dès le 12 août 1920, fait connaître à Naville sont point de vue à propos de cette nouvelle édition de la Classification des sciences. Il constate que ce livre ne reconnaît pas la relativité des connaissances. Ce simple résumé de l'expérience actuelle pourrait être détruit par l'expérience de demain. Naville admet des lois, Pareto uniquement des uniformités toujours provisoires. Il n'y a pas de lois logiques. Si un syllogisme contient quelque chose en plus des prémisses, il est expérimentalement faux. Impossible, donc, de définir d'une manière absolue le vrai, le bon, le beau, etc. On n'a que des abstractions portant ces noms, lesquelles sont relatives aux personnes qui les formulent ou qui les acceptent.

Pareto ne partage non plus les thèses de Naville sur le pragmatisme. Dans certaines circonstances et dans certaines limites, le faux peut être plus utile à la société que le vrai. Il peut être encore plus utile à la science s'il ouvre la voie à une vérité scientifique relative et provisoire. Croire à la vérité et à l'utilité d'une science aussi arriérée que la sociologie parait au sociologue de Lausanne aller trop vite en besogne. La vérité ainsi que l'utilité ne sont pas des valeurs absolues. On peut en évaluer les effets, les conséquences produites par les valeurs, mais cette évaluation est toujours relative. La tentative de Naville de concilier la science avec la philosophie et la religion ne parait pas à Pareto concluante ${ }^{79}$.

\footnotetext{
77 Sur l'apport de la linguistique saussurienne à l'économie v. J. Molino, Saussure et l'économie, «Revue européenne des sciences sociales», XXII, 1984, n. 66, pp. 145-161, et sur les contributions à la sociologie, voir l'édition critique du Cours de linguistique générale de F. de Saussure préparée par Tullio De Mauro et publiée par Laterza de Bari et par Payot de Paris.

${ }^{78}$ Naville conteste le refus de Comte et d'autres positivistes de donner la dignité de science à la psychologie et défend la thèse d'une interdépendance entre la biologie, la psychologie et la sociologie. Voir à ce propos F. Vidal, La place de la psychologie dans l'ordre des sciences, «Revue de synthèse ", tome CXV, nos 3-4, juillet-décembre 1994, pp. 327-353 et A. Petit, Quelle place pour la psychologie dans le positivisme?, Ibid., pp. 393-415.

${ }^{79}$ V. Pareto, Euvres complètes, op. cit., XIX, pp. 1040-1042 et Matériaux..., op. cit., pp. 232-234.
} 


\section{Vers une philosophie des sciences, de la morale et de la politique}

Dans le livre Classification des sciences (1920) les cinq derniers chapitres traitent de la vérité, du devoir, de l'altruisme, de l'organisation sociale et de l'organisation politique, thèmes propres à la philosophie morale et à la philosophie politique et très indirectement liés à la problématique de la classification. Ils sont à la fois une critique ou une réfutation des philosophies faisant de la science une déformation de la réalité ou encore une apologie de la science maîtresse dans son domaine, n'admettant aucune autorité supérieure à la sienne, ne reconnaissant pas ses limites, ne prenant pas conscience du mystère qui l'enveloppe et prétendant «fermer les fenêtres que l'âme humaine s'efforce d'ouvrir sur l'au-delà». En même temps, ces chapitres constituent une revendication de la justice et de la liberté individuelle, de l'égalité et des droits de l'homme. A la fin du chapitre consacré à la canonique de l'organisation sociale on peut lire: «Je dois vouloir énergiquement ma liberté, mais je dois vouloir aussi celle d'autrui! Si je la veux, ma volonté est réalisée quand autrui est libre; je suis libre parce qu'il est libre, même si sa liberté limite mon action et me cause de la gêne et des privations. Cette volonté altruiste doit inspirer le Pouvoir; elle doit inspirer aussi les particuliers et les groupes partiels. Aucune organisation sociale ne suffit pour rendre les hommes libres. La mauvaise volonté, l'égoïsme peuvent abuser de toutes les organisations pour asservir. Pour la liberté générale il faut chez la plupart la volonté que la justice règne; et le plus puissant créateur de la volonté de justice est l'amour du prochain.» ${ }^{80}$

En réalité, ces chapitres ajoutés aux essais publiés dans les années précédentes, forment un petit traité de philosophie morale et sociale, dont le but principal est de donner des bases scientifiques à la raison pratique et à la morale rationnelle et conditionnelle.

Si la morale est une discipline régulatrice des actes volontaires, si pour réaliser les idéaux elle doit s'appuyer sur une théorie des buts obligatoires et des moyens pertinents, la connaissance de la réalité et de ses lois est alors essentielle. Dans ce cas, la morale, théorie des moyens les meilleurs pour atteindre l'idéal, est un mélange de téléologie et de connaissance réelle. "La sociologie, cette science nouvelle, lui apprendra à se tromper moins souvent dans le choix de ses moyens, à faire moins souvent fausse route. Elle fondera ou du moins consolidera cet 'art social rationnel', cette 'pratique raisonnable', dont j'attends, mois aussi, beaucoup de bien, à condition que sa première assise soit la téléologie morale. $\rangle^{81}$

Bien entendu, une telle morale doit être construite sur les buts (le plaisir et la rationalité) que les hommes se donnent. La moralité est la réalisation de la vérité

${ }^{80}$ Les recherches sur l'altruisme, fondement de la coopération humaine, vont aujourd'hui dans une direction différente de celle prônée par Naville. Pour une vue synthétique v. E. Fehr \& S. Gächter, Altruistic punishment in humans, «Nature», vol. 415, 10 January 2002, pp. 137-140; E. Fehr \& U. Fischbacher, The Nature of human Altruism, Ibid., vol. 425, 23 October 2003, pp. 785-791, E. Fehr \& S,.V. Renninger, The Samaritan Paradox, «Scienific American Mind», 2004, pp. 15-21; AA. VV., The Neural Basis of Altruistic Punishment, «Science», vol. 305, 27 August 2004, pp. 1254-1258.

${ }^{81}$ La morale conditionnelle, «Revue philosophique de la France et de 1'Etranger», XXXI, 1906, II, pp. 561-575. 
relative aux valeurs et aux possibilités de les réaliser. Mais pour y arriver l'expérience ne suffit pas, «il faut de la foi, sans quoi il y aurait danger pour la vie supérieure des âmes individuelles et des sociétés $»^{82}$.

En la matière les divergences avec Pareto restent radicales. Réduire l'homme à un objet d'étude alors qu'il est surtout un sujet de devoir, placer la sociologie logico-expérimentale par-dessus la morale sociale, c'est pour Naville une approche pernicieuse. La morale sociale est le cœur battant de la vie sociale; elle peut devenir scientifique et solide, à condition de bien représenter le but social et déterminer avec quels moyens le réaliser. Dans cette perspective la sociologie est indispensable pour comprendre la nature humaine et la structure de la société, pour évaluer et pondérer les moyens nécessaires ${ }^{83}$. Dans ce sens elle est la servante de la morale.

Naville repousse également avec vigueur la thèse que la conscience enferme l'esprit du sujet en lui-même et le limite à la perception de ce qui lui est immédiatement donné. Il est convaincu que dans la pensée scientifique comme dans la pensée religieuse l'esprit entre en relation avec une réalité qui le dépasse, le domine et s'impose à lui ${ }^{84}$. A cause de cela l'homme est libre. Il peut même favoriser ou contrarier le progrès, non comme une loi nécessaire, mais comme un fait contingent susceptible de faire augmenter simultanément la différenciation dans certains domaines et d'établir dans d'autres des uniformités nouvelles. La thèse du phénoménisme est fausse. «La croyance au libre arbitre est pour moi le postulat de tous les examens de conscience comme de toutes les règles de vie ou de pensée. [...] Etre libre c'est dominer. La liberté est une domination: dominer la nature et transformer à son gré la réalité. [...] L'homme libre c'est celui qui peut vouloir comme il veut, l'homme non libre c'est celui qui ne peut pas vouloir comme il voudrait.» Précisément au nom de cette liberté, Naville s'oppose à l'Etat qui prétend d'élargir le champ de sa propre action et de «la substituer dans trop de domaines à l'action des individus et des groupes ». Ceci faisant l'initiative individuelle est anéantie, la personnalité humaine atrophiée et la contrainte sociale supprime la liberté ${ }^{85}$.

Cette fois-ci encore Pareto exprime ses divergences et remarque que Naville fait des efforts importants «pour traiter la matière dans un sens qui est opposé à celui qui suit la méthode expérimentale». Définir les sentiments, le bien, le mal, le beau, etc., est une entreprise aussi difficile que «celle de donner une forme géométrique aux nuages que chasse le vent». Naville fait «tout ce qui est possible en ce sens. Quand un esprit juste s'applique à résoudre un problème insoluble, il

${ }^{82}$ La matière du devoir. Etude analytique, «Revue philosophique de la France et de l'Etranger», XXXVI, 1911, I, pp. 113-127.

${ }^{83}$ La sociologie générale de Pareto, «Journal de Genève», 8 décembre 1919. Pareto, le 9 décembre, in Euvres complètes, op. cit., XIX, p. 1027, et Matériaux..., op. cit., p. 231, dit partager la thèse de l'importance de la morale. Cependant l'étude scientifique de la vie sociale est une activité à ne pas confondre avec l'action et avec l'agir sur la société.

${ }^{84}$ Une nouvelle philosophie de la liberté, «Bibliothèque universelle et Revue suisse», LXIII, 1911, nos 187-189, pp. 595-605.

${ }^{85}$ De quelques espèces de libertés et de quelques-unes de leurs conditions. Notes psychologiques et sociologiques, «Revue philosophique de la France et de l'Etranger», XLII, 1917, I, pp. 60-89. 
ne peut pas nous donner ce qui n'existe pas, mais il éclaire la matière et augmente par là la somme de nos connaissances.» ${ }^{86}$

Le dialogue entre le sociologue économiste Pareto et le philosophe Naville est de plus en plus difficile bien que ce dernier répète avec insistance que l'individu est à la fois un être temporel et empirique, qu'il est soumis à la causalité naturelle, que sa rationalité et son intelligibilité le rendent libre. Le devoir, la responsabilité et la solidarité s'imposent à lui en tant qu'éléments constitutifs du monde intelligible et de la morale. Le libre arbitre peut éliminer toute action causale en provenance des idées, des instincts, des habitudes, de la maîtrise de soi, et par là contribuer à garantir la liberté en l'absence de contrainte. Pour Pareto une telle conceptualisation est abstraite et spéculative, sans rapports avec les problématiques de la réalité expérimentale, avec la recherche scientifique véritable.

\section{Les derniers écrits}

Au cours de la dernière décennie de sa vie intellectuelle, le divorce de la science avec la philosophie, la rupture de plus en plus radicale entre la métaphysique, la croyance au surnaturel et l' épistémologie, inquiètent beaucoup Naville. La philosophie de Bergson lui semble ruiner la psychophysique en faisant de la conscience une invention. Attribuer à la nature de l'objet à connaître la manière spécifique de connaître, placer la gnoséologie dans la dépendance de l'ontologie, prétendre que l'intuition puisse saisir le dedans des choses, que la vie créatrice soit conçue comme la substance unique, que le négatif n'existe qu'à partir du positif, que Dieu doive être perçu et pas seulement conçu, tout cela parait inacceptable au philosophe de Genève et infliger à la recherche philosophique des graves blessures $^{87}$.

La métaphysique de Naville postule l'existence de Dieu, mais elle renonce à dire ce qu'est Dieu. Le philosophe genevois essaie de répondre à des questions du type: qu'est-ce que c'est un fait ou un objet? un événement? une existence particulière, le plaisir, la vérité, le bien de l'agent, le bien d'autrui, l'altruisme, la justice, la bienfaisance? et la liberté (pensée vraie, joie, bien en soi), l'égalité (moyen de liberté), la solidarité (ramène le multiple à l'unité, sœur de l'amour, but de liberté)?

Une attitude encore plus critique le Genevois manifeste à l'endroit de la philosophie scientiste rationaliste, pour laquelle, dit-il, l'objet de la raison n'est pas le réel mais l'intelligible et ce dernier à son tour n'est pas nécessairement réel. Les travaux d'Edmond Goblot, auteur du Traité de logique (1918) et du Système des

${ }^{86}$ V. Pareto, Euvres complètes, op. cit., XIX, pp. 962-963 et Matériaux..., op. cit., p. 227. La lettre est du 9 mai 1917.

${ }^{87}$ Voir à ce propos d'E. Boutroux, Religion et Raison, «Revue de Métaphysique et de Morale», XXII, 1914, pp. 1-16 et d'A. Naville,Comment doivent écrire les philosophes, «Le monde nouveau», $1^{\text {er }}$ mai 1923, pp. 25-28; La croyance au surnaturel et la pensée scientifique. Quelques remarques, «Revue de théologie et de philosophie», XIV, 1926, pp. 81-95; La philosophie française contemporaine d'après M. Benrubi, Ibid., XVII, 1929, pp. 28-32; La «Regina scientiarum» et sa place dans le système des sciences. A propos du livre de M. Alberto Mochi, médecin au Caire, Ibid., XVIII, 1930, pp. 184-187. Pour une vue panoramique cf. E. Castelli Gattinara, Epistémologie 1930. La tradition française, «Revue de synthèse », LXII, nos 2-3-4, avril-décembre 2001, pp. 347-365. 
sciences (1922), cantonnés dans le domaine de la raison raisonnante et de la logique déductive, sont considérés l'exemple emblématique d'une doctrine fourvoyée, faisant des lois l'objet unique de la science, de la déduction logique l'outil principal pour construire des structures et des relations abstraites. Les règles sont construites à partir de définitions, de postulats et de propositions entièrement et préalablement posés. L'interprétation de la déduction n'est que la construction d'une conséquence à partir d'une hypothèse. Dès lors le caractère nécessaire de la conséquence est impliqué par la forme des opérations. La nature de la déduction causale est assurée par le rapport entre le sujet et l'objet, par les rôles respectifs de la raison et du donné physique, des activités opératoires du sujet et des données de l'expérience.

Selon Naville cette manière de poser les problèmes est stérile, inutile, illusoire, dangereuse. En attribuant à la déduction logique une place indue, on méjuge la description et on dévalorise l'expérience des hommes et du monde social. En plus, il accuse Goblot de confondre l'ordre avec la loi, l'hypothétique avec le conditionnel, la réalité avec la possibilité, d'ignorer que les lois sont des rapports entre des antécédents et des conséquents. La composition et la structure de l'antécédent déterminent si le conséquent est l'ordre ou le désordre.

Dans ce rationalisme les faits concrets et la personnalité morale sont absents. La morale y est conçue en tant que théorie de l'inégalité des valeurs, des buts, des propensions et des tendances, sans liens avec les buts bons et obligatoires et avec les dispositions à prévenir ou à réprimer. Pour ce rationalisme la morale est celle que le milieu social conçoit et impose comme valeur ou comme normes acceptées et sanctionnées par une société «transfigurée et pensée symboliquement» et devenue une divinité selon le sociologisme de Durkheim, acceptée sans autre forme de procès par Goblot ${ }^{88}$.

La fragilité d'une telle conception est dénoncée sans ménagement. Une conduite ou une activité sont morales si elles sont conformes à la vérité. L'âme humaine est essentiellement téléologique, en elle se manifeste la nature de Dieu, d'où sa tendance naturelle à accomplir le bien. Certes, Dieu n'est pas un objet de science, il nous est impossible de le représenter. «Mais connaître sa présence en nous par l'idéal qu'il nous propose, cela n'a-t-il pas une valeur en soi, cela n'estil pas une fin pour la raison?»

La vie en société influe, assurément, sur les comportements, les conduites et les actions des agents. Néanmoins ils en restent les sujets de droits et des objets des devoirs. La collectivité, contrairement aux thèses durkheimienne, n'a pas une âme distincte de celle des individus. Le bien d'une collectivité n'est rien d'autre que le bien de ses membres. Les individus, différents et divers, sont titulaires de diverses espèces d'égalités et d'inégalités, pas toujours conciliables. Mais il est possible de les rapprocher au moyen de diverses espèces de combinaisons et c'est ainsi que l'on fixe le cadre dans lequel les inégalités de fait peuvent être perçues et reçues comme légitimes. Dans l'intérêt de l'ensemble collectif l'individu doit accepter des sacrifices et subir des contraintes momentanées. Celles-ci peuvent «aider les hommes à devenir maîtres d'eux-mêmes, et substituer la liberté du

${ }^{88}$ Un nouveau rationalisme. A propos du «Système des sciences» de M. Goblot, « Revue de théologie et de philosophie», XI, 1923, pp. 34-41. 
vouloir éclairé et profond à celle du caprice et de l'ignorance ${ }^{89}$. Pour cette raison l'égalité n'est ni un bien ni un mal; elle est un rapport entre deux termes quelconques; elle n'est pas un but, son rôle est d'être un moyen. Par contre l'inégalité est un danger pour la liberté et peut mettre en péril l'indépendance de certains individus. Doit-on la supprimer? Il est souhaitable que l'inégalité individuelle ne nuise pas au bonheur collectif. Le sort des individus doit être utile à l'ensemble, ne pas léser l'excellence de la société

Pour mieux étayer cette doctrine, Naville propose de réserver le mot intérêt aux événements psychiques, au réglage des énergies d'autoconservation en vue d'une finalité déterminée; d'employer celui de satisfaction pour indiquer le sentiment de plénitude éprouvé lorsque le résultat est atteint. De la sorte ce sentiment est également un mobile puissant d'action ${ }^{90}$.

Pareto cependant rappelle à son correspondant genevois que si l'on ne veut pas tomber dans l'empirisme le plus plat, il ne faut pas oublier que la science est analytique et que la réalité est synthétique. Elle exige des analyses complètes pourtant assez difficiles à réaliser ${ }^{91}$.

Naville reste fermement convaincu qu' «Un des meilleurs moyens pour l'individu d'être utile à ses semblables, c'est de gouverner sa propre vie avec discernement, avec prévoyance et avec suite. [...] L'individu qui donne un bon exemple est souvent plus utile aux autres que celui qui multiplie avec eux les relations et les fatigue par ses conseils. ${ }^{92}$ L'usage de l'indépendance et la résistance aux empiètements injustes favorisent, grâce aux intermédiaires psychiques, l'épanouissement de la solidarité et la plénitude de la morale. Dans cette perspective la liberté, bien suprême, est différenciée de l'activité libre consistant à faire et à obtenir ce que l'on veut. Pour les individus il s'agit d'un but légitime.

L'Etat, en donnant sa contribution au développement de ces activités libres, garantit la liberté sociale, l'ordre dans la société, le fonctionnement de l'organisation sociale, et en même temps consolide le pouvoir de commander et de se faire obéir, en d'autres termes l'autorité. «Tout homme doit servir et obéir, et tout homme aussi doit être servi et commander. Le devoir de servir c'est le sujet social, le droit de commander c'est la liberté sociale. On comprend que toute liberté

${ }^{89}$ De quelques espèces d'égalité et quelques-uns de leurs avantages ou inconvénients, «Revue philosophique de la France et de l'Etranger», XLVI, 1921, II, pp. 145-172.

${ }^{90}$ Pour davantage de précisions voir La contradiction et l' esprit humain, «Archives de psychologie», XX, 1926-1927, pp. 151-154 et Du jugement considéré soit comme acte mental, soit comme matériel logique. Critique des doctrines reçues, « Revue philosophique de la France et de l'Etranger», LIV, 1929, I, pp. 321-338.

${ }_{91}$ V. Pareto, CEuvres complètes, op. cit., XIX, p. 1145 et Matériaux..., op. cit., p. 236. La lettre porte la date du 3 mai 1923.

${ }_{92}$ De la solidarité des intérêts. Ses espèces et ses conditions, «Revue philosophique de la France et de l'Etranger», XCV, 1923, pp. 161-205. Cet essai ainsi que les articles su la liberté, l'égalité et la solidarité on été revus et modifiés et puis réunis dans le livret Liberté, égalité, solidarité. Essais d'analyse, Lausanne, Payot, 1924, 124 p. J. de la Harpe a publié un compte rendus de ce recueil dans la «Revue de théologie et de philosophie», XIII, 1925, pp. 155-157. Chez les philosophes suisses ces idées avaient un large crédit. Par exemple, Ch. Secrétan considérait la solidarité une relation forcée, un privilège à rebours grâce auquel les masses dépouillent ceux qui possèdent et détruisent ainsi les capitaux. La liberté s'épuise dans la faculté de faire le bien et de fuir le mal. Voir à ce propos M. Millioud, Ch. Secrétan sa vie et son æuvre, «Revue de Métaphysique et de Morale», XX, 1912, pp. 505-515. 
sociale implique une sujétion.» L'autorité est une relation de dépendance nécessaire. Elle est «bonne si celui qui l'exerce est plus éclairé que celui qui la subit, et s'il l'exerce en faveur de l'autre ou en faveur de l'ensemble, et non en faveur de lui-même et de son intérêt égoïste».

En conclusion, pour Adrien Naville la liberté sociale est une relation positive, différente de l'indépendance et de l'absence de relations sociales. «Elle est la possibilité d'agir sur autrui par la persuasion et dans certains cas par le commandement et la contrainte. La liberté sociale de l'un implique l'obéissance de l'autre. [...] le rapport de dépendance entre celui qui commande et celui qui obéit est bon dans la mesure dans laquelle il contribue à l'activité libre totale. [...] Les pires ennemis de la liberté sont ceux qui demandent une liberté absolue. Se contenter de peu, mais exiger énergiquement ce à quoi l'on a droit, c'est le programme libéral. [...] Aucune organisation sociale ne suffit pour rendre les hommes vraiment libres. La mauvaise volonté, l'égoïsme peuvent abuser de toutes les organisations pour asservir. Pour la liberté générale il faut chez la plupart la volonté que la justice règne; et le plus puissant créateur de la volonté de justice est l'amour du prochain.»

On est libre dans une société libre, dans une société capable de créer des liens consensuels entre les individus; dans une société où chaque individu s'efforce de promouvoir la liberté des autres et de la concevoir comme la condition afin que tous collaborent à la réalisation des fins et du bien-être communs. La religion comme aspiration à une patrie céleste commune, a nourri et nourrira la liberté, la justice et le droit à la base des normes régulatrices des rapports interindividuels et des relations entre l'Etat et les citoyens.

\section{En guise de conclusion}

Une étude exhaustive de l'œuvre d'Adrien Naville pourra être mise en route lorsqu'on arrivera à établir sa bibliographie complète, à repérer tous ses écrits dispersés ici et là, à prendre connaissance de sa correspondance. Les quelques lettres de lui que nous connaissons et les quelques lettres que lui ont été adressées par un certain nombre de correspondants, nous montrent que beaucoup de débats intellectuels, à cette époque-là, se déroulaient grâce aux relations épistolaires ${ }^{93}$. La connaissance de ces correspondances permettra de connaître de l'intérieur une doctrine présentée au moyen d'articles et de comptes rendus, jamais de façon systématique. Dans l'état actuel de la documentation, le lecteur d'aujourd'hui a une vision kaléidoscopique du travail intellectuel de Naville.

${ }_{93}$ A titre d'exemples citons les échanges épistolaires avec Franz Brentano. Est-ce que les classifications des faits mentaux de celui-ci (représentations, volitions, attentes) ont suscité la curiosité de Naville? La correspondance avec Bretano pourrait nous éclairer sur la tentative de Naville de comprendre ce qu'il y a de commun entre des activités de l'esprit en apparence disparates (percevoir une couleur, sentir une odeur, éprouver une douleur, avoir des remords, croire que 3 plus 3 font six, etc.). De quelle manière des situations se rattachent-elles toutes à la pensée et à l'esprit? Nous savons que Brentano a théorisé l'intentionalité, c'est-à-dire la représentation que vise un acte de pensée. Sur l'histoire du concept d'intentionalité voir P. Jacob, L'intentionalité. Problèmes de philosophie de l'esprit, Paris, Odile Jacob, 2005. Il faudrait également explorer les rapports du Genevois avec Benedetto Croce et voir si les recherches sur l'histoire et sur l'utilisation des concepts de cause et effet dans les recherches historiques lui ont été de quelques utilités. Dans l'état actuel de la documentation il est difficile d'en dire davantage. 
Sa classification des sciences et sa théorie de la connaissance semblent dépassées par les développements récents de la philosophie et de la sociologie des sciences et des techniques ${ }^{94}$. Par ailleurs, il est difficile de dire à présent que le projet primitif de lier la science à la philosophie et d'enchatonner les deux dans la religion, ait été réalisé vraiment. Certes, les travaux de philosophie de la science, et notamment ceux consacrés à la classification des disciplines, ont mis en lumière que la science n'est pas l'antagoniste de la philosophie, que celle-ci a un domaine d'activité différent, spécifique, rarement fermé à la progression des découvertes scientifiques, que la rationalité scientifique est compatible, à certaines conditions, avec la rationalité argumentative de la philosophie et des sciences sociales en général. Il est difficile de contester la thèse selon laquelle la religion et la science ont en commun la recherche d'invariants dans le flot des choses sensibles, la quête de l'éternel par delà l'éphémère et que cela pour la philosophie ne signifie pas autre chose que le royaume de la Raison et de la Rationalité.

On peut constater également qu'au fil des années, Naville concentre de plus en plus son attention sur un seul secteur des sciences, celui des sciences sociales, et plus particulièrement sur la psychologie et la sociologie, disciplines retenues nécessaires pour donner à la morale une théorie du vouloir et de l'action ainsi qu'une théorie des moyens susceptibles d'aider à distinguer les fins possibles de ceux qui ne le sont pas. Ce genre de recherches l'amènent fatalement vers la philosophie sociale et politique et dont le recueil Liberté-égalité-solidarité (1924) est un témoignage significatif.

Les recherches des dernières années de sa vie (il mourra en 1930) révèlent aussi que la préoccupation majeure de l'auteur genevois est davantage la clarification des concepts basilaires que la présentation d'une philosophie sociale systématique visant à réviser les principes sur lesquels se fonde la société et les relations sociales de la modernité. Ces recherches, de l'aveu même de Naville, visent plutôt à débrouiller des notions réputées primordiales, à donner des définitions précises des mots liberté, égalité, solidarité, avec «l'espoir que ce débrouillement sera utile pour l'établissement de programmes d'action moins imparfaits que ceux dont nous vivons », d'aider les hommes à «distinguer ce qui est possible de ce qui ne l'est pas». «La reconnaissance claire des impossibilités écartera l'utopie dangereuse, la reconnaissance claire des possibilités et de leurs dépendances nécessaires ouvrira à l'action féconde des chemins nouveaux.»

Mais peut-on rationaliser la polysémie de notions devenues des valeurs désormais à la base de programmes politiques, d'actions publiques, d'identités partisanes, sans risquer de créer d'autres ambiguïtés?

Naville élude consciencieusement cette problématique ${ }^{95}$. Il croit à la puissance de la raison, au sujet rationnel, aux capacités des hommes de se rendre compte du plausible et du possible, somme toute d'êtres raisonnables.

${ }^{94}$ F. Enriques, La théorie de la connaissance scientifique de Kant à nos jours, Paris, Hermann, 1938, et G. Giorello, Introduzione alla filosofia della scienza, Milano, Bompiani, 1994.

${ }_{95}$ Voir à ce propos G. Vailati, Alcune osservazioni sulle questioni di parole nella storia della scienza e della cultura, dans Scritti, a cura di M. Quaranta. II. Scritti di scienza, Bologna, Forni, 1987, pp. 49-74, ainsi que l'essai, dans le même recueil, Des difficultés qui s'opposent à une classification rationnelle des sciences, pp. 75-86. 
Mais comment les aider à bien saisir la complexité des problématiques si on détermine uniquement, de façon spéculative, les conditions à satisfaire pour acquérir des nouvelles cognitions, pour éviter les erreurs et les fausses conclusions? Si on ne précise jamais au préalable la pesée des influences sur les modalités du raisonnement, sur l'évaluation des preuves et sur la crédibilité des opinions? Peut-on négliger le fait capital que la rationalité cognitive est à la base non seulement des croyances scientifiques mais aussi des croyances sociales?

Pour sa part, Naville, surtout dans ces derniers travaux, se borne à rappeler avec insistance: «La liberté, ou du moins l'activité libre, est un but; l'activité libre c'est la pensée vraie et la joie, c'est un bien en soi. Il n'en est pas de même de l'Egalité, elle n'est pas un bien en soi, elle n'est pas, elle ne doit pas être un but, elle est un moyen de liberté. Son rôle doit être de généraliser la Liberté. Et la Solidarité? Est-elle but ou simplement moyen? La question que je pose moi-même ne laisse pas que de m'embarrasser. Pour beaucoup de métaphysiciens la Solidarité, parce qu'elle unit, est un bien en soi; elle ramène le multiple à l'unité, elle est la sœur de l'amour, elle est le but de la Liberté. Ma je doute que la science puisse la juger tout à fait de même; la science ne connaît pas d'âme collective, elle ne connaît que des âmes individuelles; ce qu'elle considère comme but ce sont les satisfactions des âmes individuelles, c'est leur activité libre. Il faudrait donc, en science sociale, placer la Solidarité à côté de l'Egalité, et en quelque sorte audessous de la Liberté, dont l'une et l'autre seraient des moyens.» ${ }^{96}$

Ce libéralisme philosophique (autonomie de l'individu respecté dans sa dignité pour autant qu'il accorde le même respect à autrui), avec sa représentation de la nature contractuelle de l'Etat, plonge, assurément, ses racines dans la tradition calviniste et dans l'histoire communale genevoises. Mais il est aussi influencé par les avatars de la première guerre mondiale, par les bouleversements politiques, sociaux et économiques provoqués par cet effroyable conflit partout dans le monde.

Que le philosophe Naville, protestant, libéral, intellectuel ouvert aux échanges culturels cosmopolites, s'engage à réfléchir sur l'éclipse des valeurs traditionnelles et sur les débordements des nouvelles classes sociales; qu'il croie, en une conjoncture si complexe et difficile, de pouvoir orienter les hommes de bonne volonté et de leur signaler les nouveaux horizons de la recherche, de la réflexion, de la moralité, quoi de plus compréhensible et respectable? Mais suffit-il de reformuler les problèmes traditionnels, de dire que les nouveaux doivent être affrontés avec courage et sens du devoir, à l'aide d'une morale sociale rigoriste, pour reconstituer les valeurs constitutives d'une civilisation broyée par la guerre et se montrant incapable de trouver une issue à la crise?

Il est malaisé de donner ici des réponses plausibles à ces questions. Un enseignement précieux toutefois nous pouvons retenir de la vigilante et diligente activité intellectuelle d'Adrien Naville. Si la morale doit rechercher l'origine et les modalités à travers lesquelles se manifestent les sentiments moraux, alors elle est du domaine de recherche de la psychologie et de la sociologie. Si par contre elle est un ensemble de modèles normatifs des conduites humaines et des actions sociales, de processus d'intériorisation des fins suprêmes des activités humaines

${ }^{96}$ Liberté-Egalité-Solidarité. Essais d'analyse, op. cit., p. 6. 
et des processus de socialisation aux relations sociales, alors la morale se rapporte à la manière de sentir et d'apprécier le bien et le mal; elle n'a aucune accointance avec la faculté de juger le vrai et le faux. Elle ne nous dit rien sur ce que nous savons. Elle nous informe que ce que nous voulons doit être accompli, si possible, selon les règles de l'éthique, de la déontologie, ou encore sans violer les normes juridiques et les droits de l'homme.

Les débats intellectuels d'aujourd'hui, hélas!, ne nous apprennent rien de plus à ce propos.

\section{UN SÉMINAIRE À PROPOS \\ DE LA SOCIOLOGIE DE LA CONNAISSANCE SCIENTIFIQUE}

La sociologie de la connaissance occupe une place très importante dans le domaine des sciences de la société. Sa problématique principale a été, et est toujours, de rendre compte des propriétés sociales de la connaissance humaine.

Cette discipline a une longue histoire. Elle occupe une place considérable dans la tradition du savoir sociologique. Certains chercheurs en font remonter les origines à Francis Bacon (1561-1626), l'auteur du Novum Organum et d'une série d'autres travaux remarquables sur les sciences. D'autres affirment que les fondements de la discipline ont été posés par Karl Marx (1818-1883) puis par les pères fondateurs de la sociologie, notamment Vilfredo Pareto (1848-1923) et Emile Durkheim (1858-1917). Par la suite, cette branche de la sociologie a été illustrée en France par Maurice Halbwachs (1877-1945), Lucien Lévy-Bruhl (1857-1939) et Georges Gurvitch (1894-1965); en Allemagne par Max Scheler (1874-1928), Karl Mannheim (1893-1947) et tant d'autres; aux Etats-Unis d'Amérique surtout par Pitirim Sorokin (1889-1968) et Robert K. Merton (1910).

Des historiens des sciences sociales contestent cette thèse et affirment que la paternité de la sociologie de la connaissance revient au philosophe positiviste autrichien Wilhelm Jerusalem (1854-1923), auquel on doit la première tentative explicite, vers 1909, d'organiser en un véritable système conceptuel la discipline, qu'il avait nommée «Sociologie des Erkennens».

Quoiqu'il en soit de cette protohistoire et de l'histoire de la discipline, il faut noter que les chercheurs depuis la fin de la première guerre mondiale - contrairement à leurs prédécesseurs du XIX ${ }^{\mathrm{e}}$ siècle surtout préoccupés de la connaissance en général - ont réservé toute leur attention aux connaissances spécifiques, aux croyances particulières, aux savoirs ponctuels. Dès lors, ils nous ont laissé une grande variété de travaux aux résultats disparates, aux schémas interprétatifs diversifiés, contradictoires parfois et qui, pour toutes ces raisons, ne suscitent ni consensus ni accords parmi les sociologues. Voilà pourquoi il n'y a pas encore une définition acceptée unanimement de la sociologie de la connaissance et de ses liens avec les autres disciplines des sciences sociales, mais également avec la philosophie, avec l'épistémologie et avec la théorie de la connaissance.

Le plus célèbre parmi les sociologues contemporains spécialistes de la sociologie de la connaissance, l'américain Robert King Merton, a l'habitude de dire que le terme «connaissance» est si vague qu'il peut couvrir des contenus fort différents, voire opposés, par exemple la conscience, la compréhension, la représentation, l'intuition, le sentiment, les sensations, et contenir aussi une multipli- 
cité de savoirs. En effet, le terme connaissance arrive à couvrir pratiquement tous les produits culturels: mythes, notions, concepts, images, modèles esthétiques, éthiques, scientifiques, etc.

Puisqu'il est pratiquement impossible de nous accrocher à une définition stable du terme de connaissance, Merton propose aux sociologues de prendre en charge avant tout les rapports existant entre la connaissance et les autres facteurs existentiels en vigueur à un moment donné, dans une société ou dans une culture déterminée. Cependant Merton n'ignore point que les rapports entre la connaissance et la société sont très complexes et compliqués. Pour cette raison, il nous invite à identifier et à différencier, dans le bloc de la connaissance, les savoirs propres aux savoirs quotidiens des savoirs savants, la connaissance ordinaire de la connaissance scientifique, les connaissances utilisées dans la vie de tous les jours, dans la vie quotidienne, de celles produites par des spécialistes, les connaissances du sens commun de celles qui sont systématisées par des pratiques disciplinaires rigoureuses.

Dans le cours de cette année, je me consacrerai exclusivement à ce dernier point, selon lequel la connaissance scientifique est un produit social mais que des secteurs de cette même connaissance échappent, en partie ou entièrement, à la détermination de la société ou de la culture.

Ceci dit, de quel type de connaissance spécifique s'agit-il? S'il y a différentes espèces et formes de connaissance, dont certaines sont plus fortement que d'autres connectées avec la structure sociale, l'analyse sociologique doit-elle en étudier la forme ou le contenu, la genèse ou l'acceptation, l'autonomie ou uniquement l'impact sur la vie sociale?

Mon propos est d'essayer d'établir si les efforts pour arriver à fonder une sociologie de la connaissance scientifique ont quelques probabilités d'aboutir à plus ou moins longue échéance. En d'autres termes, je me propose d'élucider la question suivante: si les produits de l'activité scientifique sont influencés par la société et par sa culture, s'ils subissent, comme tous les autres produits sociaux, des déterminations absolues ou relatives, comment peut-on affirmer que la science est universelle, objective, qu'elle échappe donc aux conditionnements sociaux et historiques? Mais si l'on admet l'autonomie de la science, qu'est-ce que le sociologue peut alors faire en ce domaine, qu'est-ce qu'il peut dire à son propos?

L'histoire de la sociologie nous aidera à comprendre comment les sociologues ont abordé cette problématique et de quelle façon ils ont envisagé de l'analyser. Mais avant de faire cet excursus historique, je dois vous rappeler que les sociologues ont souvent négligé, parmi toutes les connaissances spécialisées, les connaissances mathématiques en particulier, et les connaissances produites par les disciplines dites «dures » en général. Ils étaient persuadés que les sciences exactes et les sciences naturelles ne sont pas affectées par le social et par l'historicité, qu'elles ne sont pas conditionnées par les déterminants sociaux.

Que ces sciences dépendent dans une large mesure des procédés techniques dont l'homme dispose à une époque donnée et dans un cadre donné, que ces procédés techniques soient eux-mêmes subordonnés à l'évolution sociale leur paraissait peu important, car la distinction que ces mêmes sociologues établissaient entre science et organisation scientifique les débarrassaient du soucis de prendre en charge l'histoire des techniques à une époque donnée et de résoudre ainsi des problèmes très complexes. 
Certes, depuis environ six lustres, nous nous sommes occupés du travail quotidien des savants, des financements, des politiques publiques de la science, et nous avons écrits des livres et des articles sur les communautés scientifiques des savants et sur leur pouvoir de régulation de l'activité scientifique. Cependant, les travaux empiriques sur les modes de production de la connaissance dans les sciences «dures », sur la construction des théories scientifiques, sur leur diffusion et réception, sur leurs influences et emprises, ces travaux-là sont encore rarissimes, et ceci tout au moins jusqu'aux débuts des années ' 80 .

La raison de cette carence est notoire: le très fort impact sur le plan mondial de la sociologie de la science de Merton, une sociologie qui se place par ailleurs dans la droite ligne de la meilleure tradition sociologique, celle des pères fondateurs. Cette école de pensée, dominante pendant très longtemps dans la recherche sociologique, était fermement persuadée, sur la base peut-être d'un préjugé philosophique ou d'une option relevant de l'opinion commune, que la connaissance scientifique échappe à toute forme d'influence sociale, que les théories scientifiques possèdent un statut épistémologique spécial qui les soustrait à la compétence des sociologues. En vertu de ce statut, la connaissance scientifique se dérobe aux analyses des sociologues, car elle appartient aux philosophes et aux historiens. Dès lors, la sociologie de la connaissance scientifique couvre un domaine disciplinaire très différent de celui occupé par la théorie de la connaissance et par l'épistémologie des sciences. Elle ne s'occupe que des déterminants sociaux dans l'activité scientifique. Pour cette raison, elle se confond avec la sociologie de la science.

Cette position a été fortement contestée à partir des années '80, grâce aux changements advenus, notamment pendant la décennie 1960-1970, dans la recherche philosophique et dans la recherche historique. Les travaux de T.S. Kuhn, de I. Lakatos, de P.K. Feyerabend, de S.F. Mason sur l'histoire des sciences de la nature, de R. M. Young sur le darwinisme et la biologie de l'évolution, et de tant d'autres... ont fourni les matériaux et les incitations indispensables au renouvellement des travaux récents de sociologie de la science et de sociologie de la connaissance scientifique.

C'est bien sous l'impulsion de la nouvelle philosophie de la science et sous l'impulsion de l'histoire des sciences et des techniques, qu'un certain nombre de sociologues ont proclamé que la science est un savoir pareil à tous les autres savoirs, que la sociologie de la connaissance doit étudier le fait scientifique comme n'importe quel fait social. D'autres sociologues ont commencé à faire une très nette distinction entre la sociologie de la connaissance, la sociologie de la science et la sociologie de la connaissance scientifique. D'après ces chercheurs, la sociologie de la connaissance étudie les déterminés, les déterminants et les formes de la détermination constituant la connaissance en général. La sociologie de la science s'occupe surtout de la science en tant qu'institution et profession; elle s'intéresse à l'organisation des activités scientifiques, aux influences sociales sur l'activité scientifique, sur son financement, sur son mode de fonctionnement; elle s'occupe du poids que la science exerce à son tour sur la société ainsi que des influences de la société sur la science. Par contre la sociologie de la connaissance scientifique vise à expliquer sociologiquement la construction des théories scientifiques, comment elles acquièrent une autonomie par rapport au contexte de production, deviennent universelles et pour quelle raison elles sont fort différentes 
de n'importe quel autre doctrine ou fait social. La sociologie de la connaissance scientifique étudie, par conséquent, les connaissances et les savoirs produits par des savants travaillant dans des institutions se vouant à la recherche et jouissant d'une apparente autonomie, d'une indépendance relative.

Cette tripartition est fortement contestée par des chercheurs anglais et français. Pour eux il n'y a qu'une seule approche raisonnable à l'étude sociologique de la connaissance scientifique, et cette approche doit unir la philosophie, l'histoire et la sociologie. Ces chercheurs utilisent les étiquettes « Social Studies of Sciences », «Science Studies», «Wissenschaftsforschung», pour indiquer leur domaine d'étude. Mais ces étiquettes ne satisfont pas tout le monde. Les Français hostiles à la tradition sociologique classique parlent couramment d'anthropologie des sciences et des techniques, tandis que les tenants de l'ethnométhodologie et du conflictualisme continuent à utiliser l'étiquette de sociologie de la science et ignorent celle de sociologie de la connaissance scientifique.

Pour ma part, je ne tiendrai aucun compte de ces distinctions. J'essayerai de montrer de quelle façon les sociologues ont étudié la science et les connaissances produites par elle, et de voir si l'approche sociologique de la science a une pertinence et une spécificité par rapport aux approches philosophiques et historiques.

\section{PHILOSOPHIE DE LA SCIENCE, HISTOIRE DES SCIENCES, SOCIOLOGIE DE LA SCIENCE}

Avant d'entrer dans le vif du sujet, il faut que je vous donne quelques informations préliminaires.

Depuis la nuit des temps, les hommes se sont interrogés sur le fait et sur la manière de connaître, sur les mécanismes qui nous permettent de construire ou de reconstruire, d'avoir présent à l'esprit un objet réel ou vrai, concret ou abstrait, physique ou mental. Depuis les Grecs, notamment, on s'est interrogé sur la faculté des hommes à élaborer des idées, à former des concepts ou à construire des images et des représentations. Puisque connaître signifie produire en pensée, reconstituer le mode de production des phénomènes, selon la belle formule de Jean Piaget (1896-1980); être conscient de l'existence de quelque chose consiste à saisir, à décrire, à expliquer les mécanismes de cette construction ou de cette reconstruction. Mieux, selon Jean Piaget, «Le point de départ de la connaissance est constitué par les actions du sujet sur le réel».

Les philosophes, notamment depuis Platon et Aristote, ont étudié les rapports entre le sujet qui connaît et l'objet à connaître. Ils ont donné un nom à cette activité cognitive: théorie de la connaissance, ou gnoséologie. Dans leurs innombrables écrits, ils établissent une distinction très importante entre une connaissance sensible ou empirique et une connaissance intellectuelle.

La connaissance empirique est le résultat de l'action immédiate des objets sur les organes du sens (vue, ouïe, toucher, odorat, goût); elle nous procure des sensations perceptives, immédiates, dont les impressions peuvent reparaître sous forme d'images mentales. En plus de cette connaissance empirique, il y a une connaissance intellectuelle, dite aussi conceptuelle, qui résulte de l'élaboration abstraite des données de la connaissance empirique et qui est constituée d'idées ou plus précisément de concepts; ces concepts ne représentent pas des réalités concrètes, 
mais des structures générales dont chacune caractérise une catégorie d'objets réels.

A l'intérieur de la connaissance intellectuelle, un territoire dénommé «Epistémologie» a été délimité. Les spécialistes de cette discipline, les épistémologues, n'étudient pas n'importe quel savoir, mais le savoir sur le savoir, la science, sa nature, ses méthodes, sa valeur, ses conceptualisations. J'ai dit la science et non les sciences, car les épistémologues s'intéressent à la science des sciences en général; c'est-à-dire aux principes fondamentaux qui se trouvent à la base des sciences particulières, au niveau de leurs classifications, c'est-à-dire de leurs rapports de coordination et de subordination. Ces classifications, vous vous en doutez, sont très nombreuses.

La plus célèbre est celle du physicien André Ampère (1775-1836), lequel classait les disciplines en sciences de l'esprit, ou sciences noologiques, et sciences cosmologiques, ou sciences des lois physiques de l'Univers. A sa suite, on parle encore de sciences culturelles et sciences naturelles, sciences de la compréhension et sciences de l'explication, sciences formelles ou sciences réelles, ou encore sciences idéographiques (système de signes susceptibles de suggérer les objets) et sciences nomothétiques (sciences recherchant les lois).

La dernière en date de ces classifications est celle de Jean Piaget. Le Maître distinguait les sciences nomothétiques, celles qui dégagent des lois, des sciences historiques dont l'objet est de reconstituer et de comprendre le déroulement de toutes les manifestations de la vie sociale au cours du temps.

Quoiqu'il en soit, toutes ces classifications n'entament pas l'unité fondamentale de la science, c'est-à-dire d'un savoir et d'une connaissance vrais, objectifs, valides, universels, produits grâce à des méthodes spécifiques.

Les visées des historiens sont tout autres. Ils sont confrontés à l'astronomie, à la biologie, à la mathématique, à la physique, etc., et jamais à la Science. Ils parlent de sir Isaac Newton (1642-1727), d'un savant qui est à l'origine de la physique moderne, mais aussi d'un chercheur épris de spéculations et d'exégèses bibliques, d'alchimie, d'un homme particulier, singulier, spécifique, n'ayant que peu de choses en commun avec ceux qui sont venus après lui ou qui ont vécu avant ou en même temps que lui. Pour l'historien, la trajectoire de Newton est unique, particulière, et dans cette perspective, il ne peut pas la réduire à la seule vérité ou à l'objectivité des lois de la gravitation universelle et de la décomposition de la lumière.

Edgar Ascher (Problèmes du relativisme, in «Revue européenne des sciences sociales », XXVII, 1989, n. 83, pp. 87-122) affirme que l'histoire des sciences est doublement relativiste, puisque elle doit reconstruire l'époque étudiée et le faire avec les idées et les moyens de l'époque à laquelle appartient l'historien. Ce dernier «ne peut pas traiter des documents comme de simples objets d'un cabinet de curiosités ou comme produits purement littéraires. Il doit tenir compte du fait que le scientifique du passé a voulu trouver des descriptions adéquates, des propriétés pertinentes et des lois vraies au sujet des phénomènes qu'il a choisi d'étudier. Il faut donc prendre ces prétentions au sérieux et examiner si le scientifique a réussi 'dans ce qu'il a voulu faire' et essayer de comprendre les raisons de son échec éventuel» (p. 99).

La sociologie, quant à elle, envisage la question d'un tout autre point de vue. Lorsque les sociologues font de la sociologie de la connaissance scientifique, ils 
visent surtout à mettre en évidence «les corrélations entre la connaissance et les autres facteurs existentiels de la société et de la civilisation», selon la définition classique de R.K. Merton. En d'autres termes, les sociologues essayent d'étudier les rapports existant entre l'activité cognitive et le contexte social, d'établir en somme des corrélations entre les connaissances des divers milieux et les particularités de ces mêmes milieux, du contexte socio-historique.

Vous voyez bien que la sociologie de la connaissance n'a pas la visée universaliste de la philosophie de la science ni la visée relativiste de l'historiographie. Elle se situe entre les deux. Pour cette raison, jusqu'ici, nous avons échappé à maintes difficultés et à quelques paradoxes bien connus des philosophes et des historiens. Cependant, dès que nous essayons d'étudier sociologiquement la science ou les sciences, d'autres difficultés, d'autres paradoxes et contradictions nous barrent le chemin.

Comment peut-on étudier un savoir se voulant universel, la science, un savoir arraché aux temps et aux contingences, indifférents aux rapports sociaux dans lesquels les hommes sont intégrés? Peut-on affirmer que les faits scientifiques ne sont pas tributaires de l'action du milieu social? Peut-on nier que certains faits scientifiques ont une nature sociale, propre à une société particulière, façonnée par les membres d'une collectivité?

A ces questions, les sociologues donnent aujourd'hui deux sortes de réponses: l'une dite internaliste, le «programme classique ou modéré» et l'autre externaliste, le «programme fort».

Il suffit de savoir, pour le moment, que le programme classique est minimaliste: pour lui, il y a des énoncés qui sont universels (par ex., 5 x $5=25$; l'hypothèse du continu est indécidable; la vitesse est une quantité exprimée par le rapport d'une distance au temps mis à la parcourir...), et il y a des énoncés qui sont relationnels (par ex., les taux d'intérêts, la peine de mort, les rôles masculins et féminins, les croyances dans le Nirvana et dans le Karma, etc.).

Le «programme modéré » affirme que la sociologie de la science et la sociologie de la connaissance scientifique doivent s'occuper des énoncés relationnels exclusivement. Elles doivent s'interdire l'étude des énoncés universels. Elles doivent s'en tenir, en conséquence, à l'étude de l'influence des valeurs morales, de la structure sociale ou des institutions scientifiques sur la production des chercheurs. Certes, il y a une influence de la science sur la société mais aussi de la société sur la science, sur son rythme de développement, sur le choix des sujets à traiter, sur le contenu même des travaux scientifiques. Cependant cela ne met pas en danger l'autonomie de la science, ni son objectivité, son universalité, ni non plus le caractère désintéressé de l'activité du savant.

Par contre, le «programme fort» est maximaliste: il n'y a pas un savoir objectif, universel. L'universalité de la science est une illusion. Il en va de même pour les notions d'objectivité et de vérité. La science doit être traitée par la sociologie de la même façon que la magie et les autres formes de savoirs aboutissant à des connaissances. L'autonomie de la science est un leurre. Il s'agit d'une parade élaborée à une époque donnée pour des raisons politiques. Il en va de même de la distinction entre science et technique ainsi que du partage du domaine de recherche sur la science entre la philosophie, la sociologie et l'histoire.

Ces questions seront analysées plus tard. Par conséquent, je peux fermer la parenthèse et revenir à mes propos principaux : quand et comment s'est formé, à 
l'intérieur de la sociologie de la connaissance, le domaine spécifique dénommé «sociologie de la science»? Qui sont les sociologues qui ont le plus contribué à la «fabrication» de cette discipline? Quelles sont les problématiques les plus importantes mises en évidence par l'étude de la science et des activités des savants?

\section{LA SCIENCE SELON LES PÈRES FONDATEURS}

Presque tous les sociologues du XIX ${ }^{\mathrm{e}}$ siècle ont été de l'avis que la science en tant que système objectif de connaissances méthodiquement établies échappe à la juridiction de la sociologie. La science serait indifférente aux déterminations sociales, étrangère aux idéologies.

Karl Marx lui-même était de l'avis que la «vérité scientifique est toujours paradoxale au jugement de l'expérience journalière qui ne saisit que l'apparence trompeuse des choses ». Certes, les remarques de Marx sur la science sont éparpillées dans tous ses écrits. Nous ne disposons d'aucune présentation systématique et cohérente faite par l'auteur lui-même. Cela donne lieu à diverses reconstructions conceptuelles et à des interprétations disparates, voire contradictoires, à une infinité de surinterprétation.

Sans prendre position dans ce débat aux implications philosophiques et politiques certaines, une chose semble sûre: Marx partage la conception scientifique dominante en son temps. Pour lui aussi la science est le savoir par excellence. Pour lui aussi la science arrive à s'affranchir du contexte historique et des rapports sociaux caractérisant une époque donnée. En conséquence, les rapports entre la connaissance et la science, entre la science et l'histoire, entre l'objectivité et la subjectivité, ne sont pas nécessairement affectés par les déterminismes socioéconomiques ni même par les facteurs existentiels. La science est objective et universelle, même si ses utilisations peuvent être partisanes, au profit de la classe dominante. La science peut devenir un instrument au service de finalités contingentes, particulières, et ainsi contribuer au maintien de l'exploitation opérée par le mode de production capitaliste. Néanmoins la connaissance qu'elle produit est une connaissance objective, fondée sur le fait que le monde réel a un caractère matériel unitaire, que ce monde réel est la nature.

La science, selon Marx, existe indépendamment de la conscience que nous avons d'elle et des représentations que nous pouvons en donner. Elle reste toujours la source unique de cette conscience et de ces représentations. La science, connaissance de la matière, de la nature, et de la conscience que nous en avons, instrument absolument indispensable pour agir de façon efficace, ne peut être que vraie, objective, universelle.

Marx accepte, en d'autres termes, la distinction entre la science et les utilisations de la science. La science a un seul référent, un seul déterminant, la nature, tandis que les utilisations de la science sont conditionnées par la société, par la classe dominante, et en dernier lieu par le mode de production. Seule l'histoire de la philosophie peut rendre compte de l'évolution des doctrines scientifiques; alors que les utilisations de la science, dans un mode de production économique donné, doivent être expliquées sociologiquement, par le matérialisme historique.

Dans cette perspective, la science est une connaissance universelle. Ses développements et ses évolutions sont dictés par une logique tenant compte exclusive- 
ment des lois de la matière. La nature a toujours le dernier mot à dire sur la validité de la connaissance scientifique.

La science peut agir sur la nature dans la mesure où elle arrive à se conformer aux lois naturelles, à les respecter, à s'y soumettre même lorsqu'elle veut les maîtriser.

Une telle conception est très répandue dans la culture occidentale du XIX ${ }^{\mathrm{e}}$ siècle. Emile Durkheim, par exemple, la partage très largement. En effet, pour ce sociologue, la science permet de nous affranchir du monde psychologique, de la pure subjectivité. La méthode expérimentale traduit notre dépendance vis-à-vis du monde. C'est à la science de nous faire retrouver l'ordre du monde. Dans un texte de 1909, il écrit que le savant «se met au centre du monde et le fait converger vers lui (au lieu de croire qu'il y est): c'est alors l'émancipation complète: non seulement acquiescement réfléchi, mais aussi possibilité de changer le monde».

Vous savez sans doute que Durkheim a tenté d'élaborer une explication sociologique de la genèse des catégories essentielles de la pensée humaine et des formes fondamentales du raisonnement, et ceci pour fonder rigoureusement son holisme, sa conception que la société est première par rapport à toutes les autres composantes constitutives de la vie sociale.

Durkheim est convaincu que les catégories de temps, d'espace, de genre, de nombre, de cause, de force, et ainsi de suite, varient d'un groupe social à l'autre, et à l'intérieur d'un même groupe social d'une époque à l'autre. Elles dépendent de facteurs historiques, donc sociaux, même si «nous ne savons pas exactement quels ils sont, mais nous pouvons présumer qu'ils existent».

Dans une étude parue en 1985 et traduite en française en 1994, Steven Collins nous rappelle très précisément de quelle façon Durkheim, malgré une approche très sociologique de la question, est parvenu à faire des catégories des institutions transcendant les individus et à soutenir qu'elles correspondent aux propriétés universelles des choses.

Sans entrer dans les détails, bornons-nous ici à nous remémorer ce que Durkheim a écrit dans Les formes élémentaires de la vie religieuse, livre paru en 1912.

Le sociologue français, dès les premières pages de son livre, se pose la question suivante: «Mais si les catégories ne traduisent originellement que des états sociaux, ne s'ensuit-il pas qu'elles ne peuvent s'appliquer au reste de la nature qu'à titre de métaphores?... Ainsi, en tant qu'elles nous servent à penser le monde physique ou biologique, elles ne pourraient avoir que la valeur de symboles artificiels, pratiquement utiles peut-être, mais sans rapport avec la réalité».

Le fait que ces catégories soient construites avec des éléments sociaux ne nous permet pas d'affirmer qu'elles soient sans fondement dans la nature des choses. En d'autres termes, pour échapper au relativisme inhérent à l'option attribuant aux catégories et au raisonnement une origine sociale, Durkheim doit déclarer: certes, les catégories sont dégagées par le monde social, mais nous les retrouvons également ailleurs. «La société les rend plus manifestes, mais elle n'en a pas le privilège ». Des «notions qui ont été élaborées sur les modèles des choses sociales peuvent nous aider à penser des choses d'une autre nature. Du moins, si, quand elles sont ainsi détournées de leur signification première, ces notions jouent, en un sens, le rôle de symboles, c'est des symboles bien fondés. Si, par cela seul que ce sont des concepts construits, il y entre de l'artifice, c'est un artifice qui suit de près 
la nature et qui s'efforce de s'en rapprocher toujours davantage. De ce que les idées de temps, d'espace, de genre, de cause, de personnalité sont construites avec des éléments sociaux, il ne faut donc pas conclure qu'elles sont dénouées de toute valeur objective. Au contraire, leur origine sociale fait plutôt présumer qu'elles ne sont pas sans fondements dans la nature des choses ».

Durkheim affirme péremptoirement que la genèse des notions fondamentales de la pensée ou des catégories provient de la religion et, par la suite, de la Société. Mais étant donné l'unité du monde physique et social, ces notions finissent par conquérir une certaine universalité et objectivité dans la mesure où elles traduisent correctement les lois naturelles. Précisément pour cette raison, dans la conclusion de Les formes élémentaires de la vie religieuse, Durkheim écrit ceci: «Nous avons même vu que les notions essentielles de la logique scientifique sont d'origine religieuse. Sans doute, la science, pour les utiliser, les soumet à une élaboration nouvelle; elle les épure de toute sorte d'éléments adventices; d'une manière générale elle apporte, dans toutes ses démarches, un esprit critique qu'ignore la religion; elle s'entoure de précautions pour 'éviter la précipitation et la prévention', pour tenir à l'écart les passions, les préjugés et toutes les influences subjectives. Mais ces perfectionnements méthodologiques ne suffisent pas à la différencier de la religion. L'une et l'autre, sous ce rapport, poursuivent le même but; la pensée scientifique n'est qu'une forme plus parfaite de la pensée religieuse. Il semble donc naturel que la seconde s'efface progressivement devant la première, à mesure que celle-ci devient plus apte à s'acquitter de la tâche».

En d'autres termes, Durkheim affirme que la science prend la place de la religion à la suite de la croissance et de la complexification de la société ainsi que des différenciations produites par la division du travail. Ces phénomènes épurent l'activité cognitive, la libèrent des contraintes et des déterminations sociales et en font une valeur en soi et pour soi. La science est le produit de cet affranchissement, de cette libération progressive. Ses théories demeurent relativement indépendantes des influences sociales directes. Au fur et à mesure que le développement de la société devient important, les techniques d'observation des phénomènes se perfectionnent, les conceptualisations deviennent rigoureuses et formelles et de plus en plus conformes aux réalités observées. Dès lors, les résultats scientifiques sont acceptés par tous puisque conformes au monde réel et par conséquent vrais, objectifs, universels. Un jour, la science et la raison scientifique remplaceront la religion et les connaissances jusqu'ici acceptées par un acte de foi.

L'analyse sociologique de la science, dans la perspective durkheimienne, doit se limiter aux moments où les sciences se confondent encore avec les croyances religieuses, où elles ne sont pas encore affranchies des contraintes sociales. Ensuite, l'analyse sociologique prendra en compte la naissance des communautés scientifiques, l'institutionnalisation de la méthode scientifique et des techniques pour éliminer les erreurs, les préjugés et les déformations intellectuelles. Elle pourra rendre compte de l'organisation de l'activité scientifique, des rôles professionnels et de bien d'autres aspects du travail dans les laboratoires, mais elle ne pourra rien dire sur la nature de la véritable connaissance scientifique totalement indépendante du contexte social dans lequel pourtant elle s'est développée. Plus une science s'enracine dans le monde physique, se conforme et se plie à lui, plus elle devient autonome et indépendante. L'astronomie, la physique, la biologie, selon Durkheim, en sont des exemples éclatants. 
Durkheim reste un homme de son temps, comme Marx et beaucoup d'autres savants du XIX ${ }^{\text {e }}$ siècle. Pour le sociologue français, la science reste la valeur suprême de nos sociétés, elle seule nous apporte des connaissances vraies, non déchirées par les conflits sociaux. Elle constitue un savoir commun à tous, un savoir positif, donc libre de toute contrainte et de toutes déterminations. En bref, un savoir vrai, point entaché d'idéologie.

Je n'ai pas besoin de vous montrer les limites d'une telle conception, par ailleurs partagée par presque tous les sociologues jusqu'à une date très récente.

\section{SCHELER ET MANNHEIM}

Depuis l'époque de Marx jusqu'aux années '20, les sociologues ont traité abondamment de notre problématique, ils ont étudié les relations entre l'existence sociale et la connaissance ordinaire et scientifique, mais ils n'ont jamais élaboré une vision systématique des connaissances socialement conditionnées.

Je cite, pour mémoire, un des penseurs parmi les plus représentatifs de cette époque: Max Scheler (1874-1928). Ses études de sociologie de la connaissance, traduites en français par Sylvie Mesure, se trouvent désormais réunies dans le volume Problèmes de sociologie de la connaissance (Paris, PUF, 1993).

Scheler soutient que les valeurs ont une existence objective et constituent la matière grâce à laquelle le sujet est en mesure de préférer «le bon au mauvais et le meilleur au moins bon». Il affirme aussi, contre la conception kantienne, la primauté de la valeur sur le devoir. En effet, il déclare que nous sommes dans un cosmos de valeurs que nous ne devons pas produire mais seulement reconnaître et découvrir, par l'intuition, le sentiment, la visée émotionnelle, en d'autres termes par une activité extra-théorétique, par une «mathématique du cœur» capable de saisir en stricte logique les essences des choses.

Scheler établit une hiérarchie des valeurs: sensorielles, vitales (noble/ vulgaire), spirituelles (le génie), esthétiques (beau/laid), éthico-juridiques (juste/ injuste), religieuses (sacré/profane), spéculatives (vrai/faux). Selon cette conception, les faits mentaux sont des faits d'expérience phénoménologiques distincts des faits pragmatiques, naturels et scientifiques. Ces derniers sont le produit de la «libre contemplation», de la technique et de l'expérimentation. Ces deux derniers facteurs ont appris à la science «à limiter son intérêt pour chaque aspect de la nature à la dimension quantitativement mesurable du monde et aux lois de l'enchaînement spatio-temporel des phénomènes selon leurs ressemblances et leurs différences, autrement dit: à ce qui se peut saisir comme dépendant des phénomènes possibles du mouvement» (p. 145). Fille de l'union entre la philosophie et l'expérience du travail, la science fonctionne grâce au modèle de la causalité et à une certaine théorie dynamique de la matière, «tous les deux anthropomorphiques » et donc incapables de «s'ancrer ni dans des lieux ponctuels de l'espace ni dans des points déterminés du temps objectifs... Leurs points d'ancrage devraient être à la fois extérieurs à l'espace et au temps, puisque ces forces doivent pour leur part expliquer et rendre compréhensible avant tout la matière dont, dans la sphère du phénomène objectif, se mettent en place les déterminations spatiotemporelles de la matière et de ce qui survient, ainsi que les relations qui s'éta- 
blissent sous ce rapport» (p. 220). En conséquence, «la science positive ellemême conduit à des problèmes indubitablement métaphysiques» (p. 221).

La place réservée à la science dans une telle sociologie est subalterne. Le sociologue doit analyser les facteurs réels et les facteurs idéels de la science mais l'étude des types de connaissances produites est du ressort du philosophe.

Le premier sociologue qui a travaillé ce domaine d'étude de façon systématique et exhaustive est un Hongrois de culture allemande, Karl Mannheim, né en 1893 à Budapest, professeur à l'Université de Francfort, d'où il a été chassé par les nazis en 1933. Depuis cette date et jusqu'à sa mort en 1947, il enseigna la sociologie à la London School of Economics and Political Science.

Mannheim est considéré comme le père fondateur de la sociologie de la connaissance moderne. Ses travaux en la matière sont importants et nombreux. Ses idées et ses cadres conceptuels ont constamment évolué de l'époque de Strukturanalyse der Erkenntnistheorie, ouvrage paru en 1922, aux Essays on the Sociology of Knowledge, publiés en 1952 et suivis des Essays on the Sociology of Culture, également posthumes, parus en 1956.

Une reconstruction analytique de sa pensée montre que sa sociologie, élaborée à une époque agitée et cruelle, celle des totalitarismes, ne constitue pas un système fermé ou achevé. La sociologie de Mannheim est traversée par diverses influences: le relativisme socio-historique, la doctrine de la mise en perspective, le principe weberien de compréhension et de conciliation ainsi que la doctrine de la marginalité, qui permettrait, selon son auteur, d'obtenir un décentrement et fournirait les moyens d'échapper à l'aliénation inhérente au conformisme social.

Ici, je ne vous parlerai que des idées de Mannheim à propos de l'étude sociologique de la science. Pour ce sociologue, en cela disciple de Max Weber et fidèle tenant de la tradition académique allemande, il faut nettement distinguer les méthodes et les concepts des sciences de la nature des méthodes et des concepts des sciences sociales et historiques. Les phénomènes naturels et les rapports existant entre eux sont constants et invariables. Ils sont statistiques et intemporels. Nous les étudions comme étant extérieurs à nous, comme des objets indépendants de l'esprit, objectifs; nous le faisons de façon détachée, impartiale, à l'aide d'instruments, de mesures vérifiées et contrôlées, et en recourant à nos sens, à nos facultés sensorielles qui nous permettent d'éprouver les impressions produites par les objets matériels. L'étude du monde matériel ainsi faite produit des données empiriques immuables et universelles, constamment vérifiables et validées par différents chercheurs. Ces données sont par conséquent dotées de tous les critères de vérité.

Une théorie est vraie dans la mesure où elle n'est pas contraire, pas différente, pas dérogatoire, aux objets matériels, extérieurs à l'observateur, autonomes et indépendants par rapport à sa construction théorique. Dès lors pour Mannheim la connaissance produite par les sciences de la nature demeure permanente, uniforme, objective, universelle, vraie.

Ceci étant, Mannheim ajoute aussitôt que les sciences de la nature se développent plus ou moins linéairement car elles corrigent progressivement les erreurs et, par conséquent, s'approchent constamment de la vérité. Cette dernière est conçue comme un jugement valide et vrai pour autant que l'expérience le vérifie et n'entre pas en contradiction avec lui ou avec la théorie qui le formule et le construit. 
De façon synthétique, on peut dire que pour Mannheim la connaissance scientifique évolue régulièrement en accumulant vérités externes, ou empiriques, et vérités internes, ou logiques de coordination, sur un monde physique intrinsèquement stable.

Par contre, la société et la culture ne sont pas extérieures à nous comme l'est la nature, d'où l'impossibilité de les observer avec détachement, de nous décentrer par rapport à elles, de regrouper nos observations en série, de soumettre à mesure ou à estimation les faits culturels et les faits sociaux.

Pour catégoriser des phénomènes culturels, pour en saisir la signification, il faut interpréter la pensée des individus, le sens que les agents donnent à leurs actions. La pensée n'est pas observable à la manière des objets matériels, des objets du monde réel. Chaque individu, chaque groupe social a des valeurs et des sens spécifiques. En plus, ils varient d'une époque historique à l'autre, d'un groupe social à l'autre, d'un segment de ce même groupe à un autre segment. Le sociologue a aussi des valeurs; il partage celles de son groupe d'appartenance. Il peut difficilement s'en décentrer, se détacher de son monde d'origine. Il peut, certes, avoir une compréhension sympathisante de ce qu'il étudie, mais il ne peut jamais le considérer comme étant extérieur à lui. Son système de référence, par rapport auquel il apprécie et analyse les comportements ou les actions des acteurs sociaux, reste toujours à la base de son travail.

Pour toutes ces raisons, Mannheim considère qu'aucun produit culturel n'est intemporel et immuable. Tous les phénomènes socio-culturels sont variables et en perpétuelle mutation, d'où le dynamisme de leurs significations, d'où la difficulté de cumuler les recherches dans les sciences historiques et sociales.

Dans ces sciences, il faut tenir compte des spécificités, des particularités, des différences, des changements. A chaque époque d'une société, à chaque stade d'un système culturel, il faut recommencer le travail. Le travail antérieur peut nous servir à comprendre les sociétés du passé, rarement les nôtres, celles dans lesquelles nous vivons. Pour ces raisons les sciences physiques, selon Mannheim, ont une nature assez spécifiques, dont la sociologie ne peut aucunement faire l'économie. Pour lui, les facteurs existentiels, les conditionnements sociaux sont marginaux, subalternes dans ces sciences. La genèse historico-sociale d'une idée scientifique, les conditions qui ont rendu possibles son développement et sa vérification dans une organisation, dans un laboratoire, dans un organisme de recherche, n'ont aucun effet sur le contenu, sur la forme et la validité d'une théorie, sur sa vérité, sur son objectivité, sur son universalité. Les erreurs et les errements d'aujourd'hui seront corrigés demain. Ils seront aussitôt oubliés par les savants, même si les historiens continueront à les raconter dans leurs livres. La distinction faite entre les sciences de la nature et les sciences sociales et historiques permet à Mannheim d'affirmer que les premières échappent à l'analyse sociologique tandis que les secondes en constituent la matière principale.

Cette affirmation constitue-t-elle vraiment le noyau dur de la sociologie de Mannheim?

Il m'est difficile de répondre à cette question par un oui. Mannheim a beaucoup écrit et dans ses textes on peut trouver des passages où il insinue le doute qu'en physique, aussi, la stabilité des structures catégorielles n'est pas définitivement établie. A ce propos, les exemples fournis par les recherches de la physique quantique ou de celle des hautes énergies lui paraissent emblématiques. Dans 
cette physique-là, il n'est pas certain que la connaissance scientifique soit intemporelle et immuable. Il faut reconnaître cependant que sur ce thème, Mannheim a laissé des notations rapides, jamais vraiment développées par une argumentation solide. Si nous voulons rester fidèles aux textes fortement construits, nous devons alors admettre, voire reconnaître que le problème épistémologique central de la sociologie de Mannheim, comme par ailleurs de celle de Durkheim, est surtout celui de la relativité, ou comme les deux l'appellent: le relationnisme.

Selon cette épistémologie sociologique, toute pensée sociale, comme par ailleurs n'importe quelle forme de pensée extérieure aux sciences exactes, est relative à une position sociale donnée; elle est conditionnée par une perspective singulière, particulière, par l'intérêt de classe. Dans le domaine de la pensée sociale, il n'y a pas de critères universaux (des concepts et termes universels applicables à toutes les cultures et à tous les individus de toutes les sociétés) pour fonder la validité d'une assertion spécifique, caractéristique, sui generis. Et même la sociologie de la connaissance n'échappe guère à cette contrainte.

Il a été objecté à Mannheim que si cela était fondé, nous ne disposerions d'aucun moyen pour valider une thèse quelconque, y compris celle qui affirme que la connaissance sociale est déterminée ou conditionnée par la société.

La parade de Mannheim à cette objection est bien connue. Il l'a formulée à peu près ainsi: les assertions des sciences de la culture sont de nature et de forme très différentes de celles des sciences naturelles, toutefois elles peuvent aussi nous fournir des connaissances vraies. Et pour rendre plausible cette thèse, Mannheim doit réviser tacitement certaines de ses propositions méthodologiques sur les sciences de la nature. En effet, dans le livre Idéologie et Utopie, paru en 1919 mais accru en 1929, il écrit que la méthodologie des sciences physiques, expérimentales et formelles est un produit dérivé de la «Weltanschauung», d'une vue métaphysique du monde, sous-jacente à la conception que la bourgeoisie en ascension se fait de la vie. Dans cette conception du monde de la classe bourgeoise, conception pétrie par l'idéologie du cosmopolitisme démocratique, la place de la connaissance personnelle, qualitative, intuitive, est dévalorisée d'une manière constante. Le statut de connaissance authentique est réservé exclusivement aux formulations validables universellement. Ainsi, on arrive à éliminer les connaissances produites par les classes subalternes, on peut taxer d'opinions personnelles ou partisanes tout ce qui n'est pas produit par la culture dominante, celle de la classe hégémonique dans le mode de production capitaliste. C'est l'hégémonie des bourgeois, leur domination sociale et politique, qui fait prévaloir la prééminence, dans la vie intellectuelle, de la connaissance scientifique et l'épistémologie élaborée à partir de celle-ci. Et c'est ainsi que tous les savoirs et toutes les connaissances sont mesurés et évalués à l'aune d'une connaissance imposée par l'idéologie d'une classe. Cette connaissance ayant la prétention d'être reconnue par tous comme étant la seule et unique valable et vraie.

L'influence du marxisme sur Mannheim est évidente. Cependant ce sociologue en fait un usage original et hétérodoxe. En effet, il s'emploie à élaborer une épistémologie alternative, a-marxiste, capable de donner la dignité de savoir aux sciences sociales.

Comment le fait-il? En partant du présupposé qu'il y a un monde physique et un monde social. Les sciences de ces deux mondes sont garanties par deux épistémologies différentes. La prétention de liquider les sciences sociales au nom de 
l'épistémologie des sciences dures, n'est rien d'autre, selon Mannheim, qu'une mystification idéologique. Tout en reconnaissant aux sciences dures une validité certaine, Mannheim admet que celles-ci également, dans certaines circonstances, sont conditionnées par le point de vue adopté par l'observateur-chercheur. Pour cette raison la vieille épistémologie positiviste, celle de la nature des choses, est devenue inadéquate, inappropriée. Il faut réviser cette épistémologie ainsi que la vieille notion d'objectivité, selon laquelle la dite objectivité n'est que la qualité de ce qui existe indépendamment de l'esprit.

Mannheim ne conteste point le fait qu'on puisse arriver à des conclusions objectives à propos d'un phénomène donné, que ces conclusions puissent être vérifiées par d'autres au moyen de procédures et d'instruments préliminairement définis. Mais il est de l'avis que ces conclusions «objectives» restent incomplètes, vu qu'elles ont été produites suite à l'adoption d'un point de vue ou d'une perspective spécifique. Pour cette raison, les conclusions changeront lorsqu'un contexte social nouveau fera surgir de nouveaux points de vue et des nouvelles perspectives. Il y a objectivité lorsque les chercheurs partagent le même point de vue de départ, utilisent les mêmes instruments de mesure, effectuent les expérimentations avec les mêmes protocoles. Si les chercheurs adoptent des schémas de référence différents, alors l'objectivité ne pourra être obtenue que de façon indirecte.

Vous avez compris que Mannheim considère que l'objectivité reste indissociable de l'accord intellectuel, du consensus sur les points de vue, sur les instruments et sur les protocoles de recherche; et surtout, il est de l'avis que l'accord-consensus est possible à condition que les résultats produits par une certaine perspective puissent être transférés dans une autre perspective, ou être compatibles entre eux.

Pour Mannheim il y a des systèmes de référence divergents, surtout dans les périodes historiques de transition. Il arrive donc qu'un savant doive choisir entre des points de vue opposés ou des perspectives différentes. Comment fait-il un tel choix?

En donnant la préférence à la perspective qui lui paraît la plus large, la moins contestable logiquement, celle qui lui semble capable d'embrasser le plus de données; en somme la perspective la plus compréhensive et la plus féconde.

Par ce biais Mannheim croit liquider la vieille épistémologie positiviste, celle de la correspondance entre les propositions théoriques et la réalité du monde observé, l'épistémologie de la correspondance entre les énoncés déduits et la réalité extérieure, celle selon laquelle toute théorie déductive ou inductive doit se rapporter à un objet, aux données contrôlables par les sens.

Ainsi Mannheim, tout en reconnaissant aux sciences dures la capacité de produire des connaissances objectives, place ces dernières à l'intérieur d'un contexte socio-historique donné. Le changement du contexte modifie l'objectivité, car un nouveau point de vue l'a conditionnée ou la conditionnera.

Vous vous attendez peut-être, à ce point, que je vous dise que Mannheim a abandonné sa doctrine, d'origine néokantienne, de la connaissance scientifique en tant qu'ensemble de vérités universelles et statiques. Vous attendez, peut-être, que je vous dise aussi que la connaissance du monde physique et celle du monde social dépendent du type de questions que nous formulons à leur sujet, des finalités que les sujets cognitifs se fixent et des situations socio-historiques dans lesquelles ils 
agissent. Hélas! je dois vous détromper. Mannheim se révolte à l'idée que la connaissance scientifique puisse être envisagée comme socialement conditionnelle, contingente, semblable à la connaissance socio-historique. Pour lui, les deux types de connaissance ne sont pas analogues. Il s'agit de connaissances distinctes, parallèles, disposant d'épistémologies différentes. Le relationnisme de la science physique reste pour lui un cas spécial du principe général du relationnisme. Mannheim suggère que la connaissance produite par les «social scientists» est en étroite connexion avec la position sociale qu'ils occupent et le contexte social dans lequel ils agissent. Par contre, la connaissance produite par les chercheurs en sciences naturelles subit un seul conditionnement, celui $\mathrm{du}$ temps et de l'espace.

D'un certain point de vue, Mannheim affirme que la connaissance physique et la connaissance sociale sont intrinsèquement limitées et susceptibles de révisions; et d'un autre point de vue que la nature de ces deux connaissances n'est pas la même et qu'elles subissent des déterminations, des conditionnements, des contraintes fort dissemblables.

En conclusion, Mannheim a dit et répété que l'épistémologie positiviste ne concerne que les sciences de la nature, qu'elle est inadaptée aux sciences sociohistoriques. Il a essayé d'élaborer une épistémologie appropriée aux sciences sociales, une épistémologie relationnelle, capable de rendre compte des déterminismes sociaux et existentiels agissant dans le monde. Selon lui la vieille épistémologie n'est plus susceptible de rendre compte des problématiques de la physique des quanta et des hautes énergies, mais elle ne peut pas encore être abandonnée. En attendant, la connaissance scientifique est différente pour lui de la connaissance sociale: la première dispose d'une logique indifférente aux conditionnements existentiels, tandis que la seconde est déterminée par la société et par sa culture, par la condition et la position de classe des acteurs sociaux.

Position étrange et contradictoire, en vérité, que celle de Mannheim. Malheureusement, les sociologues n'ont retenu qu'une seule thèse de son enseignement: la science est un domaine étranger à l'analyse sociologique.

\section{LA SOCIOLOGIE DU SAVOIR}

Ernst Grünwald a publié en 1934 un volume intitulé Das Problem des Soziologie des Wissens. Versuch einer kritische Darstellung der Wisssenssoziologischen Theorien (Wien-Leipzig, Braumueller, 1934; nouveau tirage, Hildesheim, Olms, 1967) par lequel il a voulu montrer que les difficultés de la sociologie de la connaissance et de la sociologie de la science dérivent de l'incertitude ou de l'ambiguiité de leurs postulats de base. Ces postulats peuvent essentiellement être ramenés, selon Grünwald, à deux visions du monde.

Les sociologues qui adhérent à la première, donnent pour acquis le fait que l'homme ne peut être compris en dehors du contexte historico-social dans lequel il vit. Ses pensées et ses idées proviennent du contexte historico-social qui les détermine, les conditionne, les façonne. A l'inverse, les sociologues qui adhérent à la seconde vision du monde demeurent convaincus que l'homme est partie intégrante de la nature, que la connaissance provient exclusivement de l'expérience empirique et que l'erreur est un éloignement de l'expérience empirique produit 
par des intérêts sociaux. Toutefois, l'erreur est toujours susceptible de correction, à condition bien évidemment que nous soyons capables de revenir à l'expérience et de nous soumettre à ses impératifs.

Le premier postulat est de nature historiciste: l'historicisme rejette les approches selon lesquelles les présuppositions et la structure de la connaissance sont fondées sur une théorie universelle de la nature humaine; l'historicisme insiste sur le fait que la connaissance est fondée localement et située historiquement. Suivant cette approche, le monde doit être considéré comme historiquement changeant.

La nature du deuxième postulat est psychologico-naturaliste. L'homme est partie intégrante du monde naturel. La connaissance ne peut se baser que sur l'expérience empirique de ce monde naturel. Le fait de ne pas se soumettre à l'expérience empirique est à l'origine de l'erreur. Il est possible que des intérêts sociaux nous poussent à négliger ou à mépriser l'expérience, cependant la rectification et la correction restent toujours possibles à condition de revenir à la source de toutes les vérités, à l'expérience acquise des faits naturels, du monde réel.

Ces deux façons de concevoir l'homme et la nature sont à l'origine de deux traditions: celle pour qui la science est relative à une société donnée et qu'elle constitue, de ce fait, un savoir parmi d'autres; et celle selon laquelle la science est un savoir spécifique, singulier, parce que constamment soumis à l'expérience immédiate (le premier contact de la pensée avec l'univers matériel), à l'expérience physique (qui consiste à agir sur les objets pour en découvrir les propriétés qui sont alors abstraites de ces objets comme tels), puis à l'expérience scientifique par laquelle tout est corrigé grâce à l'assimilation des choses à la raison, assimilation réalisée par abstraction à partir des coordinations entre les actions.

Par conséquent, d'un côté nous aurions le savoir social soumis aux conditionnements existentiels, à la société, et de l'autre le savoir scientifique soumis aux conditionnements et aux déterminismes physiques, à la nature. Le premier savoir serait changeant, variable, local, particulier, tandis que le deuxième serait permanent, stable, global, universel. Certes, ce dernier savoir peut être dégradé par des conditionnements sociaux, mais il peut être rectifié, comme je l'ai déjà dit. Il peut donc être rétabli en son entièreté, alors que le premier est indéfectiblement inséparable du contexte social qui lui a donné la vie.

En d'autres termes, Grünwald veut démontrer que tous les sociologues qu'il étudie dans son livre doivent être rattachés à l'une ou l'autre de ces traditions, et que c'est bien leur type de rattachement qui détermine leur vision de la science en tant que savoir relatif parmi d'autres savoirs relatifs ou en tant que savoir singulier, spécifique, auto-organisateur, autonome, universel.

La position de Grünwald est, je crois, trop tranchée et intellectualiste. Peu de sociologues peuvent être rattachés sans autre à l'une ou à l'autre des deux traditions dont il est question dans son livre Le problème de la sociologie du savoir.

La séparation entre l'approche historiciste et l'approche psychologico-naturaliste n'est jamais aussi nette lorsqu'on est engagé dans la recherche de terrain. Marx, par exemple, n'est pas systématiquement le chercheur historiciste que Grünwald décrit. Durkheim et Pareto n'affichent pas systématiquement leur naturalisme ou leur psychologisme naturaliste. Tous ces sociologues, et Talcott Parsons l'a bien montré aux débuts des années ‘40, croient que l'histoire a un sens et une direction, qu'il y a des lois de l'organisation de la vie sociale, qu'il est 
possible de connaître ces lois en leur constance et uniformité, et que la science est le seul moyen à notre disposition pour les connaître ou les découvrir. Ces mêmes sociologues croient fermement que le contexte social peut voiler la véritable connaissance et que la tâche de la science consiste précisément à déchirer les voiles qui la dissimulent.

Cette croyance n'est plus celle des sociologues d'aujourd'hui. Les sociologues d'aujourd'hui ne croient plus à l'existence de lois de l'histoire, à l'histoire comme progrès, au sens et aux fins de l'histoire, aux soleils de l'avenir, à une société sans conflits et sans aucune inégalité. Les sociologues contemporains veulent plutôt savoir pourquoi certains contextes sociaux limitent substantiellement la capacité des hommes à agir et pourquoi ces limitations changent dans le temps et dans l'espace. Ils veulent savoir aussi quelles sont les implications de ces mêmes limitations. Et il se demandent également s'il y a un secteur de l'activité humaine, par exemple la science, qui échappe aux contraintes du contexte social. De ce point de vue-là, le livre de Grünwald n'est qu'un témoignage intéressant sur la culture sociologique de la fin des années ' 20 du siècle passé.

\section{LA SYSTÉMATISATION DE STARK}

Il est incontestable que la sociologie de la connaissance scientifique de Mannheim a joué un rôle important, en éloignant l'analyse sociologique de l'étude des sciences de la nature. On s'en rend compte en lisant les travaux de Werner Stark (né en 1909 et actif surtout à partir de 1947). Stark est l'auteur d'un ouvrage monumental The Sociology of Knowledge. An Essay in Aid of a Deeper Understanding of the History of Ideas, paru en 1958 (nouvelle éd., London, Routledge and Kegan Paul, 1967), et d'une Sociology of Religion en trois volumes, publiée à partir de 1966. En 1963, il fait paraître un essai The fundamental Forms of social Thought (New York, Fordham University Press) dans lequel on trouve une présentation systématisée de son approche de la sociologie de la connaissance.

Stark ne discute même pas le point concernant les rapports entre la science et l'analyse sociologique. Pour lui, il va de soi que la science est foncièrement étrangère aux analyses sociologiques. Stark ne fait que reprendre les thèses de Mannheim tout en s'employant à les étayer et à mieux les argumenter. Son but est de consolider pour de bon la thèse selon laquelle la science échappe à l'analyse sociologique ou, en d'autres termes, aux conditionnements existentiels.

Les conceptions de Stark sont exposées ex professo dans sa Sociologie de la connaissance. Ce livre est constitué de deux parties. Dans la première, il essaye de délimiter le champ de la sociologie de la connaissance tel qu'il s'est peu à peu formé, au cours du dernier siècle, grâce aux travaux des chercheurs; tandis que la deuxième partie énumère et discute les problèmes actuels étudiés et débattus par les spécialistes. L'ouvrage constitue un état de la question très précis, tel qu'on pouvait le dresser à la fin des années ' 50 .

Bien qu'il ne soit pas aisé de faire un résumé de ce gros livre, écrit en anglais mais pensé en allemand, je vais essayer tout de même d'en soutenir la gageure.

Dès les premières pages de son travail, Werner Stark insiste sur le fait que le monde physique se caractérise par sa permanence et par sa stabilité. Cela permet aux savants d'observer les phénomènes avec précision et de la manière la plus 
complète possible. Les physiciens arrivent à établir une correspondance constante entre la nature toujours invariante et leurs propres formulations théoriques. Dès lors, l'accord intellectuel entre les physiciens est relativement aisé. Cette conformité de pensée, ce consentement généralisé entre les membres de la même communauté de chercheurs, sont le produit de l'objectivité foncière de la connaissance scientifique.

Il n'en va pas de même dans toutes les sciences. Il y a une différence de taille et de nature entre les savants qui étudient le monde physique et ceux qui analysent le monde social. Les faits sociaux sont fabriqués par des individus socialement et historiquement situés, alors que les faits naturels nous sont donnés, ils sont là. Nous sommes inclus dans les faits sociaux, tandis que les faits naturels sont extérieurs à nous.

Après avoir soutenu qu'il y a une correspondance directe entre la véritable connaissance scientifique et les phénomènes naturels invariants, et que cette correspondance n'est jamais affectée par des facteurs socio-historique, Stark ajoute une deuxième thèse qu'il formule plus ou moins ainsi: la connaissance du monde physique est cumulative et fondée. Cette connaissance est élaborée dans le cadre d'une perspective visant à produire le maximum de résultats avec le minimum d'efforts, en vue d'obtenir une grande efficacité technique. En d'autres termes, Stark affirme que la science doit tenir les comptes des valeurs économiques et technologiques propres à une époque. Dès lors, la connaissance scientifique est liée à une perspective particulière, à une attitude spécifique, elle est animée par un souci de rendement, par l'efficacité. Ceci étant, elle ne peut donc être ni stable, ni permanente. D'une certaine manière, elle est aussi conditionnelle, contingente.

Stark écarte cette objection en ripostant que depuis l'aube de l'humanité, la valeur fondatrice de la science a toujours été la même: dévoiler les mystères du monde naturel, découvrir les lois de la nature, en maîtriser les processus, les soumettre à sa volonté. Les savants n'ont pas d'autres alternatives devant eux. En conséquence, l'efficacité technique de la science reste un facteur marginal, secondaire, non nécessaire.

Le contenu factuel de la science est donc inaccessible à l'investigation scientifique, à l'enquête sociologique, car il ne s'agit jamais d'un cas d'espèce, d'un événement historique, mais toujours de données universelles. Le contenu factuel de la science est le produit de l'application cumulative de la même perspective à un monde naturel constitué ab aeterno.

Une telle conception de la connaissance scientifique a des implications très complexes. J'en mentionne quelques-unes.

La première, je la formulerais ainsi: si le corpus des connaissances scientifiques s'est constitué par accumulations successives au fil des siècles, il ne peut y avoir alors de changement de signification dans la base factuelle de la science. La véritable connaissance scientifique peut être formulée d'une seule et unique manière. Le corpus des connaissances s'accroît, certes, de plus en plus, d'année en année, mais sa base ne subit aucune mutation, aucune rectification, ni ajustement, ni calibrage.

La deuxième implication est celle-ci: les découvertes scientifiques sont très différentes des découvertes culturelles et artistiques, de celles que l'on peut observer dans le monde social. La découverte scientifique ne consiste jamais à 
produire de nouvelles significations, comme c'est toujours le cas dans les arts ou en philosophie. La science doit reconnaître des réalités existantes, en dévoiler les secrets. L'astronome, dit Stark, essaye de comprendre les données disponibles; ces données lui préexistent. Il doit s'y soumettre, se laisser conduire par elles. Le savant reste esclave de la vérité objective, de la réalité, tandis que l'homme de culture et le philosophe expriment toujours et surtout les valeurs qui leur sont chères. Bien entendu, Stark sait que les données scientifiques peuvent être conceptualisées et interprétées de façon différente et à des niveaux de généralisation disparate. Il sait aussi que certains niveaux de généralisation et certaines conceptions ne sont connectés que très indirectement aux observations empiriques. Mais à vrai dire, il en fait peu de cas. Il s'agirait de l'appendice métaphysique de la science, d'une extrémité inoffensive, anodine, aisément dissociable de la science véritable.

Comment départager la science véritable de son appendice métaphysique?

La réponse de Stark est assez simple et claire: la science s'interroge sur ce que sont les choses et comment elles fonctionnent, alors que la métaphysique est toujours en quête du pourquoi. La science ne se demande pas, par exemple, pourquoi il y a une accélération constante de la chute des corps. Elle ne pose pas la question du pourquoi de la théorie de l'évolution des espèces. Elle se borne simplement à constater que la formulation théorique concorde avec les observations.

Je pourrais objecter, à ce propos, que la théorie de Darwin a été inspirée par l'analyse de la dynamique sociale de Malthus, donc qu'elle a été fortement influencée par des facteurs sociaux, mais Stark jugerait assurément cette objection peu pertinente, donc irrecevable. Pour lui, la théorie de Darwin est absolument vraie, elle synthétise un nombre important d'observations empiriques. Elle n'est ni révisable, ni corrigible. Elle n'est d'aucune manière influencée, disons de façon significative, par un facteur social quelconque.

Stark écrit à ce propos qu'il s'agit d'un point de doctrine universellement accepté par tous les sociologues de la connaissance scientifique. Et il cite Marx, Lukacs, Mannheim, Alfred Weber et Merton qui auraient - à quelques nuances près - une même vision de la science. C'est possible, car ils étaient tous profondément imprégnés de la même philosophie standard de la connaissance scientifique. Une telle philosophie, exclusive et dominante pendant très longtemps dans les milieux académiques et intellectuels, n'envisageait qu'une seule position possible en matière de connaissance scientifique et d'activité intellectuelle. Et cette position reconnaissait, sans autre forme de procès, que la science se développait dans un monde où les problèmes existentiels n'avaient aucune place.

Je dois aussitôt reconnaître que la façon dont la sociologie de la connaissance scientifique a été élaborée, jusqu'à une époque récente, est tributaire de cette conception standard de la science, de cette philosophie qui postule que le monde est réel, objectif, préexistant, inflexible aux préférences et aux intentions de ses observateurs. Cette philosophie suppose que les caractéristiques de ce monde peuvent être tout à fait fidèlement représentées. La science est alors l'activité intellectuelle par laquelle nous décrivons exactement les objets, les processus et les relations qui font partie du monde des phénomènes naturels. Une bonne connaissance scientifique valide dévoile et révèle ces phénomènes et les incorpore à ses schémas théoriques. 
Certes, dans le monde naturel il y a des mutations et des mouvements uniformes, variés, divergents, convergents, rayonnants; cependant ce même monde naturel est caractérisé par des uniformités immuables, des régularités, des constantes. Bien entendu, nous pouvons nommer «lois permanentes et universelles » ces uniformités, ces régularités empiriques, et cela pour une raison bien simple: elles nous disent ce qui arrivera en tout lieu, en toute circonstance, en tout temps. L'observation objective fournit les matériaux grâce auxquels les lois naturelles peuvent être correctement formulées. L'observation objective est assurée par les procédures expérimentales, par les contrôles, par une série de méthodes et de pratiques qui empêchent les déformations, les erreurs, les méprises des sens, et même les illusions produites par les préjugés ou les aveuglements engendrés par nos vanités et nos susceptibilités.

Que le corpus de la connaissance scientifique soit foncièrement empirique est un fait indiscutable. Cependant, ce même corpus contient aussi des généralisations dépourvues de lois d'observation, de lois ne pouvant pas être vérifiées. Ces propositions abstraites, spéculatives, jouent un rôle important dans le système de pensée scientifique. Ces propositions aident à comprendre des régularités observées et encore dépourvues de lois; elles coordonnent des lois d'observation se trouvant dans des systèmes de référence séparés, et parfois elles aident à révéler des phénomènes observables et jusque-là inconnus. Il arrive que ces propositions abstraites, ces spéculations, deviennent des lois d'observation grâce au développement des techniques expérimentales. Mais en attendant, ces propositions spéculatives n'ont pas le même statut que les lois d'observation, elles ne représentent pas le monde naturel de façon aussi rigoureuse que ce qu'exige la science. Il arrive que ces propositions, utiles pendant quelque temps, doivent être abandonnées parce qu'elles sont fragiles, contradictoires, parce qu'elles sont devenues inutiles. Le diablotin de James Clerk Maxwell (1831-1879), provisoirement utile à la théorie électromagnétique de la lumière; l'éther, dont on considérait qu'il remplissait le vide et servait de support à la propagation des ondes lumineuses; le phlogistique considéré lui aussi, pendant longtemps, comme un des matériaux de la composition des corps, sont des exemples probants de cet état de fait. Par contre, le contenu des lois d'observation n'est déterminé que par le seul monde physique.

Stark, comme par ailleurs presque tous les sociologues de la connaissance scientifique avant lui, ne distingue pas les lois d'observation des lois théoriques.

Les lois théoriques peuvent être révisées, abandonnées, corrigées, tandis que les lois d'observation jamais. Lorsqu'une loi théorique génère des inférences non confirmées par l'observation, elle est revisitée, révisée, ajustée aux faits, ou encore délaissée au profit d'une hypothèse alternative, ou de différentes hypothèses parmi lesquelles on choisira la plus solide, celle qui semble la plus plausible. L'hypothèse ainsi retenue devient alors une loi théorique. Les faits que l'ancienne loi théorique expliquait sont alors soumis à la nouvelle loi théorique et, grâce à celle-ci, ils sont mieux reliés les uns aux autres et ils offrent un schéma conceptuel plus performant.

Pour conclure sur ce point, je dirais que Stark a raison et tort en même temps: il a raison de soutenir qu'il y a des lois universelles, il a tort de ne pas distinguer les lois d'observation des lois théoriques. Il a raison de dire que les lois des sciences de la nature ne sont pas influencées par la société; il a tort de ne pas ajouter que cela est vrai pour les lois d'observation et l'est beaucoup moins pour 
les lois théoriques. Ces dernières peuvent dériver d'une idéologie, d'une métaphysique dominante, d'une croyance en vogue. Le contenu des spéculations théoriques peut varier, car il n'est pas déterminé par les données de l'observation et peut donc être conditionné par des intérêts sociaux.

Il n'en reste pas moins que la connaissance des théories présuppose celle des faits; et celle des faits présuppose celle des théories. En d'autres termes, toute connaissance repose sur des règles, qui sont elles-mêmes normatives. Je veux dire par là que toute connaissance n'est pas réductible à du savoir positif. La science positive - la physique par exemple - est indépendante du temps et du lieu. Elle a affaire à des phénomènes reproductibles et prévisibles: la même expérience peut être répétée, à tout moment, dans n'importe quel laboratoire. De tels critères ne sont pas applicables à n'importe quelle espèce de connaissance: ni celle du passé - l'histoire - ni celle de la signification d'un texte - la critique littéraire - par exemple, ne peuvent être assimilées à des sciences positives. Il y a des sciences qui ne peuvent pas formuler d'énoncés prédictifs ni être testées en laboratoire. Mais cela ne veut pas dire qu'elles ne constituent pas une forme de connaissance. Si l'on admet l'existence de plusieurs formes de connaissance, alors les thèses de Mannheim reprises par Stark sont pertinentes. Si nous affirmons que la connaissance est une, alors ces thèses sont fausses et le problème du conditionnement social et existentiel de la science reste sans solution.

\section{LES THÉORICIENS DES IDÉOLOGIES}

Je dois très rapidement et très sommairement faire état, ici, des contributions offertes par les spécialistes de la théorie des idéologies à l'approfondissement de la théorie de la connaissance sociologique de la science et des sciences.

Vous savez que les sociologues appellent idéologie l'ensemble des idées et des images présentes dans des écrits ou dans des discours, mais aussi l'ensemble des comportements communs à une masse d'individus, voire à des institutions. L'idéologie permet au sociologue de comprendre le mode d'agir des hommes, les justifications qu'ils en donnent et qui le légitiment, c'est-à-dire les actions sociales et les systèmes de valeurs qui les fondent.

Précisément pour cela, notamment depuis le début du siècle, les sociologues ont essayé de nous dire d'où viennent les idéologies et à quoi elles servent. Il est entendu que les réponses à ces questions varient en fonction des écoles sociologiques, bien que certaines d'entre elles suscitent davantage d'adhésions que d'autres. Le marxisme, par exemple, a plus fortement marqué l'étude des idéologies que d'autres courants de pensée.

Pour les sociologues marxistes, l'origine des idéologies se trouve dans l'illusion d'une fausse conscience, dans les intérêts matériels des classes sociales, dans un mode particulier de production économique et de rapports sociaux, le mode de production capitaliste. Pour d'autres sociologues, l'origine des idéologies se trouve dans les passions et les sentiments humains, ou encore dans la nécessité d'argumenter des choix arbitraires, des choix qui sont à la base de n'importe quel système de valeurs, à la base de toutes les cultures.

Pour les sociologues marxistes, les idéologies assurent la domination d'une classe sociale; elles servent à situer les groupes sociaux dans les luttes politiques 
et sociales. Certes, elles sont des productions imaginaires, qui ne peuvent nous donner une véritable connaissance de la réalité. Pour certains marxistes, dès lors, il y a conflit entre la connaissance scientifique et la connaissance idéologique, tandis que pour d'autres, une telle distinction est irréelle dans les sociétés capitalistes où tous les savoirs, toutes les formes de connaissance sont contaminés par les intérêts de classe, donc affectés idéologiquement.

Pour les sociologues non-marxistes, les idéologies intègrent les individus à un groupe social. Elles les aident à percevoir et à lire la réalité sociale, à désigner leurs amis et à identifier leurs ennemis, à intérioriser les valeurs à poursuivre et à lutter pour stabiliser ou encore pour transformer une organisation, un groupe social ou une société. Certains de ces sociologues, critiques par ailleurs du marxisme, réhabilitent le rôle des idéologies. Pour eux, elles sont des états de conscience, des représentations, des discours pour justifier ou contester des choix politiques. Elles fournissent les liens entre les représentations discursives, les argumentations et les passions humaines, les sentiments.

Louis Dumont, pour ne citer que lui, affirme que les idéologies édifient la réalité sociale. D'après ce sociologue comparatiste français, les idéologies constituent des formes de connaissance pratique de la société. Il n'y aurait pas de société sans une ou des idéologies. Pour lui, l'idéologie est l'ensemble social des représentations, des idées et des valeurs communes à une société. Par exemple, l'idéologie économique est à la base de l'individualisme, donc de l'idéologie moderne qui constitue l'ensemble des représentations communes de notre civilisation.

$\mathrm{Si}$ les idéologies dissimulent ou masquent les intérêts pratico-politiques, ou si elles sont liées aux passions et aux désirs, aux nécessités de l'action, de la vie et de l'agir en société, quel est leur rapport avec la science et avec le travail des scientifiques?

Pour Marx et Engels, la structure économique de la société conditionne toutes les pratiques sociales, toutes les pensées, tous les sentiments, toutes nos manières de penser et de sentir. Cependant, et je l'ai déjà dit en son temps, ce conditionnement n'entraîne pas une relation nécessaire, inéluctable. Conditionnement ne signifie pas déterminisme: principe selon lequel, une fois que les conditions d'existence d'un phénomène sont posées, celui-ci doit inéluctablement se produire.

Je vous ai aussi déjà dit que selon Marx et Engels l'idéologie, contrairement à la connaissance scientifique, ne peut fournir qu'une connaissance fausse de la réalité, une connaissance imaginaire, une connaissance qui falsifie les vrais problèmes et les perspectives du monde réel. L'idéologie est un rapport vécu et imaginaire que les hommes établissent avec leurs conditions d'existence. A ce titre, elle est un savoir explicatif de la réalité historique et sociale, elle peut fournir des explications compréhensibles et simples des faits et des événements de notre monde, mais elle reste une forme de connaissance biaisée, de méconnaissance de la réalité sociale et de l'action historique, contrairement à la science qui est un savoir explicatif, critique, vérifié, testé à l'aide de procédures expérimentales constamment remises en cause.

Contrairement à l'idéologie, la science n'est pas une lecture directe du réel, ni même une image ou une photographie de ce même réel. La science est un savoir relatif et provisoire, alors que l'idéologie est un savoir à prétentions absolues et définitives. 
L'exemple le plus significatif de cette séparation entre la science et l'idéologie nous est offert par Vilfredo Pareto (1848-1923). Pour ce sociologue, l'idéologie masque toujours un programme, dissimule des intérêts, nous mystifie en nous faisant croire qu'un jugement de valeur est un jugement de fait. La science, par contre, est connaissance des choses, des faits. L'idéologie se fonde sur des absolus invérifiables, la science sur des constatations toujours contrôlables. La science est problématique et provisoire. L'idéologie est un savoir indiscutable et figé. La science est hypothétique, conjoncturelle; elle ne fait que poser des problèmes dont elle recherche la solution. C'est une connaissance sans sujet connaissant. Pour cette raison, la science s'accroît sans que nous en ayons immédiatement conscience.

Pour Pareto, la nature de la science est très différente de la nature de l'idéologie. La première avance par approximations successives tandis que la seconde fait appel à l'absolu, à l'indémontrable. La science est démonstration, critique, dépassement; l'idéologie est certitude, conviction, cristallisation. Une théorie scientifique peut être transformée en idéologie. Dans ce cas, elle n'est plus tenue à l'objectivité, au devoir de conformité avec les faits; sa seule obligation devient celle de l'utilité sociale. La finalité de la science est la vérité, celle de l'idéologie l'utilité. La science postule que le monde réel existe indépendamment de la connaissance que nous en avons, que nous pouvons connaître ce monde réel grâce à des procédures d'enquête, que la connaissance est vraie pour autant qu'elle s'approche du monde réel ou qu'elle est isomorphe à la structure de la réalité. Pour ces raisons, la sociologie ne doit pas étudier la connaissance certifiée en tant que telle, mais plutôt les conditions sociales favorisant la poursuite d'une connaissance objective. Au cours de cette étude, le sociologue devra mettre en évidence les idéologies qui rendent difficile cette recherche de connaissance et les opérations effectuées pour contourner les obstacles.

Les idées de Pareto seront reprises par d'autres et contribueront plus tard à consolider la conception standard de la connaissance scientifique. Je pense au travail réalisé par Gérard De Gré (né en 1915, professeur d'anthropologie et de sociologie, dès 1968, à l'Université de Waterloo, dans l'Iowa) pour faire accepter l'approche sociologique de la connaissance dans les Universités américaines. La démarche de De Gré a été magistralement décrite par François Chazel dans son article intitulé L'institutionnalisation de la sociologie de la connaissance aux Etats-Unis : l'apport de Gérard De Gré (paru dans la «Revue française de sociologie», XXVIII, 1987, pp. 663-677).

Cette approche de l'étude empirique de la science met surtout en évidence les modèles normatifs les plus stables des savants et néglige la signification sociale de leurs engagements cognitifs, pourtant constamment changeants. Cette approche, n'est pas indifférente aux facteurs économiques, technologiques et militaires. Tout en réservant une bonne place aux normes et aux valeurs de l'homme de science, à la nature de l'ethos scientifique, elle revendique avec rigueur la séparation de la science et de l'idéologie, de la science de la société. D'après cette approche, l'analyse scientifique ne doit rien avoir en commun avec l'analyse sociale, et donc avec les idéologies.

Sorokin, le maître de Merton et de Parsons, amorcera un changement dans la tradition qui sépare nettement l'analyse scientifique de l'analyse socio-historique. Mais de cela nous traiterons dans le séminaire de l'année prochaine. 